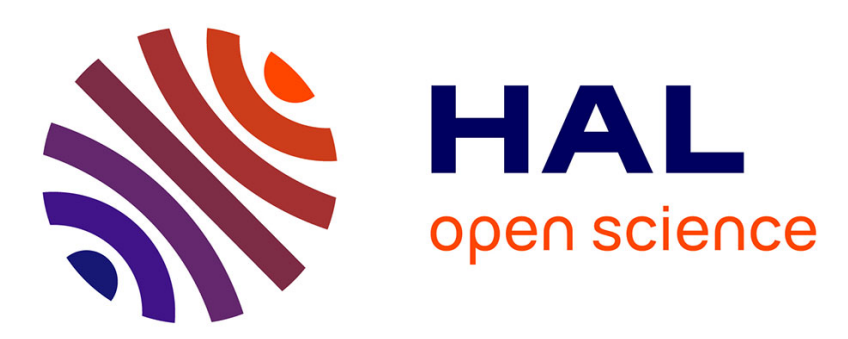

\title{
Stabilized DDFV schemes for Stokes problem with variable viscosity on general $2 \mathrm{D}$ meshes
}

\author{
Stella Krell
}

\section{To cite this version:}

Stella Krell. Stabilized DDFV schemes for Stokes problem with variable viscosity on general 2D meshes. Numerical Methods for Partial Differential Equations, 2011, 27 (6), pp.1666-1706. 10.1002/num.20603 . hal-00385687v2

\section{HAL Id: hal-00385687 \\ https://hal.science/hal-00385687v2}

Submitted on 15 Feb 2010

HAL is a multi-disciplinary open access archive for the deposit and dissemination of scientific research documents, whether they are published or not. The documents may come from teaching and research institutions in France or abroad, or from public or private research centers.
L'archive ouverte pluridisciplinaire HAL, est destinée au dépôt et à la diffusion de documents scientifiques de niveau recherche, publiés ou non, émanant des établissements d'enseignement et de recherche français ou étrangers, des laboratoires publics ou privés. 


\title{
STABILIZED DDFV SCHEMES FOR STOKES PROBLEM WITH VARIABLE VISCOSITY ON GENERAL 2D MESHES
}

\author{
STELLA KRELL*
}

\begin{abstract}
Discrete Duality Finite Volume" schemes (DDFV for short) on general meshes are studied here for Stokes problems with variable viscosity with Dirichlet boundary conditions. The aim of this work is to analyze the well-posedness of the scheme and its convergence properties. The DDFV method requires a staggered scheme, the discrete unknowns, the components of the velocity and the pressure, are located on different nodes. The scheme is stabilized using a finite volume analogue to Brezzi-Pitkäranta techniques. This scheme is proved to be well-posed on general meshes and to be first order convergent in a discrete $H^{1}$-norm and a discrete $L^{2}$-norm for respectively the velocity and the pressure. Finally numerical experiments confirm the theoretical prediction, in particular on locally refined non conformal meshes.
\end{abstract}

Key words. Finite-volume methods, Stokes problem, DDFV methods, variable viscosity.

1. Introduction. This paper is devoted to the finite volume approximation of the $2 \mathrm{D}$ steady Stokes model with variable viscosity:

$$
\begin{aligned}
-\operatorname{div}(2 \eta(x) \mathrm{Du})+\nabla p & =\mathbf{f}, \quad \text { in } \Omega, \\
\operatorname{div}(\mathbf{u}) & =0, \quad \text { in } \Omega,
\end{aligned}
$$

where $\mathbf{u}: \Omega \mapsto \mathbb{R}^{2}$ is the velocity, $p: \Omega \mapsto \mathbb{R}$ is the pressure and Du $=\frac{1}{2}\left(\nabla \mathbf{u}+{ }^{t} \nabla \mathbf{u}\right)$ is the symmetric part of the gradient of $\mathbf{u}$. We assume that the viscosity $\eta$ may in fact explicitly depend on the space variable. Note that in physical models, the viscosity depends on other characteristics of the flow like density, temperature, through the coupling with other equations. Nevertheless, solving a problem like (1.1) is the first step needed towards the approximation of more complex models.

Finite volume methods have been extensively study to solve problem (1.1) with constant viscosity: $-\eta \Delta \mathbf{u}+\nabla p=\mathbf{f}$. Hence, in this case, after integration of the equation on each control volume, we only need to approximate the normal component of $\nabla \mathbf{u}$ on the interface between two adjacent control volumes (see, for instance, [20, 21] for methods on admissible and conformal meshes), whereas for variable viscosity, the presence of the symmetric part of the gradient Du imposes to address the problem of the reconstruction of the full velocity gradient on the whole domain, even for admissible and conformal meshes. We propose here a staggered method: the discrete unknowns, the components of the velocity and the pressure, are located on different nodes. The most celebrated staggered scheme is the MAC scheme $[23,31]$ on cartesian grids. Actually, for a cartesian grid and constant viscosity, the scheme we propose here is equivalent (except on the boundary) to two uncoupled MAC schemes written on two different staggered meshes. Moreover, even in the case when the viscosity is a constant, a possible outflow boundary condition of physical interest is to impose the normal component of the stress tensor on the boundary. In that case, we also really need to deal with the original formulation (1.1) of the problem which makes appear the total stress tensor $2 \eta \mathrm{Du}-p \mathrm{Id}$, where Id denotes the identity matrix. Hence, from a numerical point of view we need a discretization of $\mathrm{Du}$ in order to deal with this problem. Although we will only consider here the case of Dirichlet boundary conditions, we emphasize the fact that our framework naturally allows to take into account those outflow boundary conditions.

Different methods of gradient reconstruction for cell-centered finite volume have been proposed since the last ten years, one can refer to [3], [12], [17], [19] and [15, 25]. In all

*Université de Provence, Laboratoire d'Analyse, Topologie et Probabilités, 39 rue F. Joliot-Curie, 13453 Marseille Cedex 13, France (krell@cmi . univ-mrs.fr) 
cases, the crucial feature is that the summation-by-parts procedure permits to reconstruct the whole two dimensional discrete gradient, starting from two point finite differences. Many of them have been compared in the benchmark of the FVCA5 conference [24], for scalar diffusion problems.

We consider here the class of finite volume schemes called "Discrete Duality Finite Volume" (DDFV for short). The DDFV schemes have been first introduced and studied in $[15,25]$ to approximate the solution of the Laplace equation on a large class of $2 \mathrm{D}$ meshes including non-conformal and distorted meshes, without "orthogonality" assumptions as for classical finite volume methods. Those schemes require unknowns on both vertices and "centers" of control volumes. These two sets of unknowns allow to reconstitute two-dimensional discrete gradient (defined on new geometric elements called diamonds) and discrete divergence operators that are in duality in a discrete sense (see Theorem 3.1). The number of unknowns doubles compared to usual cell-centered finite volume schemes, but the gradient approximation becomes simple and quite efficient. The benchmark [24] brings out that the DDFV method is a competitive first order method especially as far as the accuracy of the gradient is concerned. The DDFV framework is thoroughly recalled in Section 3.

Since ten years, the DDFV strategy has then been applied for several linear and nonlinear problems: linear anisotropic diffusion equations in $[6,15,25,26]$; convection-diffusion problems in [10]; div-curl problems in [14]; the nonlinear diffusion equations for Leray-Lions operators in $[2,5]$. We can also mention [11] where the DDFV method is adapted to solve numerically a bi-domain problem arising in bio-mathematics.

Concerning the DDFV discretization of the Stokes problem we are interested in here, the first results can be found in [13] where the author first considered the natural extension of the DDFV scheme classically used for the Laplace problem, that is: velocity unknowns located at both vertices and centers of control volumes and pressure unknowns at the diamond cells (those cells where the discrete gradient operator is defined). Unfortunately, the corresponding scheme is only proved to be well-posed for particular classes of meshes. Indeed, the wellposedness result relies on a uniform discrete inf-sup condition, which is still an open problem for general meshes.

To overcome this difficulty, we propose here to add to this scheme a stabilization term in the mass conservation equation. This stabilization term is inspired by the well known BrezziPitkäranta method [8] in the finite element framework. This stabilization strategy have been successfully used in the finite volume framework [20,21], since it is easy to write and to implement, while preserving a good accuracy. We prove that the stabilized DDFV scheme is well-posed for 2D general meshes. Moreover, this stabilization term plays a key role in proving error estimates. Indeed, the appropriate choice of the stabilization term enables to prove a stability result (see Theorem 6.1) which is the first step towards the error estimates. More precisely, we prove here a first order convergence for the velocity, for its gradient and for the pressure in the $L^{2}$-norm provided that the exact solution satisfies usual regularity assumptions.

Note that, an alternative strategy has also been proposed in [13] to overcome the difficulties of the analysis of the non-stabilized scheme. The author proposed to formulate the Stokes problem in the vorticity-velocity-pressure form and then to approximate the velocity on the diamond cells and the pressure on both vertices and centers of primal control volumes. This approach uses the fact that $\Delta \mathbf{v}$ is equal to $\nabla \operatorname{div} \mathbf{v}-\operatorname{curl} \operatorname{curl} \mathbf{v}$, for any vector field $\mathbf{v}$. Thus, it seems that it can not be easily generalized to the case where the viscosity is variable.

Only the 2D case is on purpose in this article. 3D extensions of DDFV schemes have been proposed in [11, 1], [27] and [9] for linear and nonlinear anisotropic diffusion equations, see Remark 3.4, and the extension of the present work is proposed in [30]. 
Outline. This paper is organized as follows. Section 2 is dedicated to introducing basic notation. In Section 3, we recall the DDFV framework. In Section 4, we introduce the DDFV stabilized scheme for the Stokes problem (2.1) and prove its well-posedness (see Theorem 4.1). In Section 5, we present the main results of discrete functional analysis necessary for the theoretical study of the finite volume method. These results include properties of the discrete strain rate tensor, in particular we prove a discrete Korn inequality (see Theorem 5.1). In Section 6, we study the stability properties of the approximate solution with respect to the data (see Corollary 6.1). Then in Section 7, we prove error estimates provided that the exact solution lies in $\left(H^{2}(\Omega)\right)^{2} \times H^{1}(\Omega)$ (see Theorem 7.1). Finally, in Section 8, theoretical error estimates are illustrated with numerical results. In the concluding Section 9, we discuss the extension of our study to some fully practical variants of the finite volume scheme and to even more general viscosity, for instance discontinuous viscosity, see [29].

2. Stokes model. We are concerned with the finite volume approximation of the Stokes equations with variable viscosity: Find $\mathbf{u}: \Omega \rightarrow \mathbb{R}^{2}$ and $p: \Omega \rightarrow \mathbb{R}$ such that:

$$
\begin{aligned}
\operatorname{div}(-2 \eta(x) \operatorname{Du}+p \mathrm{Id}) & =\mathbf{f}, \quad \text { in } \Omega, \\
\operatorname{div}(\mathbf{u}) & =0, \quad \text { in } \Omega, \\
\mathbf{u} & =\mathbf{g}, \quad \text { on } \partial \Omega, \quad \int_{\Omega} p(x) \mathrm{d} x=0 .
\end{aligned}
$$

For the sake of simplicity, we restrict the presentation to the case of Dirichlet boundary conditions and regular right-hand sides. We assume that $\Omega$ is a bounded connected polygonal domain in $\mathbb{R}^{2}, \mathbf{f}$ is a function in $\left(L^{2}(\Omega)\right)^{2}$, the viscosity $\eta$ is a function in $L^{\infty}(\Omega)$ and $\mathbf{g}$ is a function in $\left(H^{\frac{1}{2}}(\partial \Omega)\right)^{2}$ which verifies the compatibility condition:

$$
\int_{\partial \Omega} \mathbf{g}(s) \cdot \overrightarrow{\mathbf{n}} \mathrm{d} s=0 .
$$

The viscosity $\eta: \Omega \rightarrow \mathbb{R}$ in (2.1) is supposed to be Lipschitz continuous on the whole domain $\bar{\Omega}$ and bounded: there exists three constant numbers $\mathrm{C}_{\eta}, \underline{\mathrm{C}}_{\eta}, \overline{\mathrm{C}}_{\eta}>0$ such that

$$
\underline{\mathrm{C}}_{\eta} \leq \eta(x) \leq \overline{\mathrm{C}}_{\eta}, \quad \text { for a.e. } x \in \Omega,
$$

and

$$
\left|\eta(x)-\eta\left(x^{\prime}\right)\right| \leq \mathrm{C}_{\eta}\left|x-x^{\prime}\right|, \quad \forall x, x^{\prime} \in \bar{\Omega} .
$$

The well-posedness of the problem $(2.1)$ is studied in $[33,7]$ for a constant viscosity. Thanks to a Korn inequality, it can be generalize to variable viscosity. In order to study convergence rates of our approximate solution, we need to assume regularity of the solution $(\mathbf{u}, p)$ of the problem (2.1). If $\Omega$ is a convex polygon, $\mathbf{g}$ is equal to zero and the viscosity is constant, then in $[28,33]$ the regularity of the solution is the following

$$
\mathbf{u} \in\left(H^{2}(\Omega)\right)^{2} \text { and } p \in H^{1}(\Omega) .
$$

We denote by $\mathcal{M}_{m, n}(\mathbb{R})$ the set of real $m \times n$ matrices (we note $\mathcal{M}_{n}(\mathbb{R})$ when $m=n$ ). In the sequel, $\|\cdot\|_{2}$ stands for the natural $L^{2}$-norm when we consider scalar valued and vector valued functions and for the Frobenius norm when we consider matrix valued functions:

$$
\begin{aligned}
\|\xi\|_{\mathcal{F}}^{2} & =(\xi: \xi), \quad \forall \xi \in \mathcal{M}_{2}(\mathbb{R}), \\
\|\xi\|_{2}^{2} & =\int_{\Omega}\|\xi(x)\|_{\mathcal{F}}^{2} \mathrm{~d} x, \quad \forall \xi \in L^{2}\left(\Omega, \mathcal{M}_{2}(\mathbb{R})\right),
\end{aligned}
$$


where $(\xi: \widetilde{\xi})=\sum_{1 \leq i, j \leq 2} \xi_{i, j} \widetilde{\xi}_{i, j}=\operatorname{Trace}\left({ }^{t} \xi \widetilde{\xi}\right), \quad \forall \xi, \widetilde{\xi} \in \mathcal{M}_{2}(\mathbb{R})$.

REMARK 2.1. The matrix norm $\|\cdot\|_{\mathcal{F}}$ satisfies the following property

$$
\left\|\frac{A+{ }^{t} A}{2}\right\|_{\mathcal{F}} \leq\|A\|_{\mathcal{F}}, \quad \forall A \in \mathcal{M}_{2}(\mathbb{R}) .
$$

\section{The DDFV framework.}

\subsection{The meshes and notation.}

The meshes. We recall here the main notation and definitions taken from [2]. A DDFV mesh $\mathcal{T}$ is constituted by a primal mesh $\mathfrak{M} \cup \partial \mathfrak{M}$ and a dual mesh $\mathfrak{M}^{*} \cup \partial \mathfrak{M}^{*}$. An example for square locally refined primal mesh is on Figure 3.1.
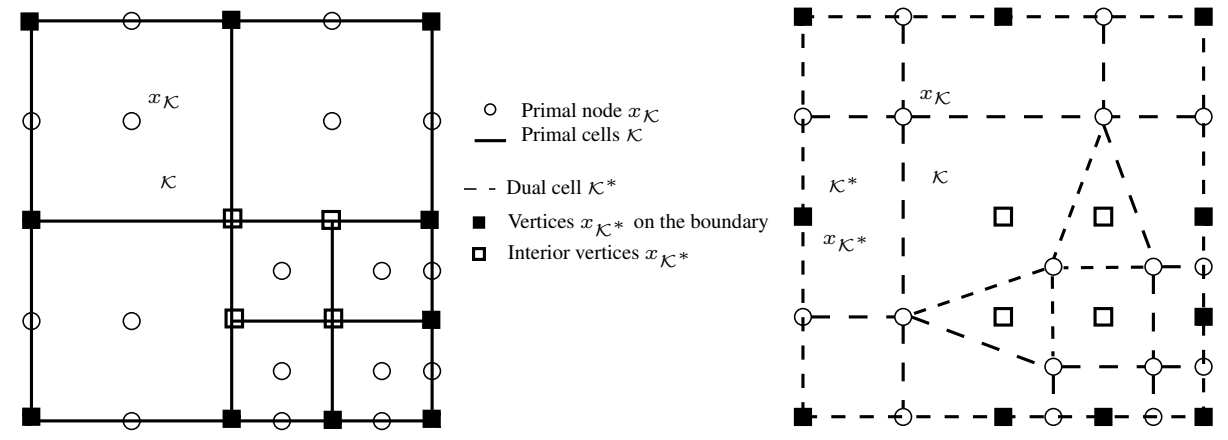

FIG. 3.1. The mesh $\mathcal{T}$. (Left) The primal mesh $\mathfrak{M} \cup \partial \mathfrak{M}$. (Right) The dual mesh $\mathfrak{M}^{*} \cup \partial \mathfrak{M}^{*}$.

The primal mesh $\mathfrak{M}$ is a set of disjoint open polygonal control volumes $\mathcal{K} \subset \Omega$ such that $\cup \overline{\mathcal{K}}=\bar{\Omega}$. We denote by $\partial \mathfrak{M}$ the set of edges of the control volumes in $\mathfrak{M}$ included in $\partial \Omega$, which we consider as degenerate control volumes. To each control volume and degenerate control volume $\mathcal{K} \in \mathfrak{M} \cup \partial \mathfrak{M}$, we associate a point $x_{\mathcal{K}}$. For each degenerate control volume $\mathcal{K} \in \partial \mathfrak{M}$, we choose the point $x_{\mathcal{K}}$ equal to the midpoint of the control volume $\mathcal{K}$. This family of points is denoted by $X=\left\{x_{\mathcal{K}}, \mathcal{K} \in \mathfrak{M} \cup \partial \mathfrak{M}\right\}$.

Let $X^{*}$ denote the set of the vertices of the primal control volumes in $\mathfrak{M}$ that we split into $X^{*}=X_{i n t}^{*} \cup X_{\text {ext }}^{*}$ where $X_{i n t}^{*} \cap \partial \Omega=\emptyset$ and $X_{e x t}^{*} \subset \partial \Omega$. With any point $x_{\mathcal{K}^{*}} \in X_{i n t}^{*}$ (resp. $x_{\mathcal{K}^{*}} \in X_{\text {ext }}^{*}$ ), we associate the polygon $\mathcal{K}^{*} \in \mathfrak{M}^{*}$ (resp. $\mathcal{K}^{*} \in \partial \mathfrak{M}^{*}$ ) whose vertices are $\left\{x_{\mathcal{K}} \in X\right.$, such that $\left.x_{\mathcal{K}^{*}} \in \overline{\mathcal{K}}, \mathcal{K} \in \mathfrak{M}\right\}$ (resp. $\left\{x_{\mathcal{K}^{*}}\right\} \cup\left\{x_{\mathcal{K}} \in X\right.$, such that $x_{\mathcal{K}^{*}} \in \overline{\mathcal{K}}, \mathcal{K} \in$ $(\mathfrak{M} \cup \partial \mathfrak{M})\}$ ) sorted with respect to the clockwise order of the corresponding control volumes, This defines the set $\mathfrak{M}^{*} \cup \partial \mathfrak{M}^{*}$ of dual control volumes. In some particular cases, we can have an overlap of dual cells, like it is shown Figure 3.2. To eliminate such cases, we made Assumption 3.1.

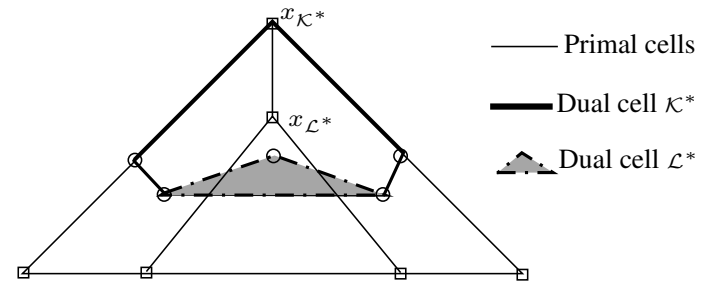

FIG. 3.2. An example where two dual cells $\mathcal{K}^{*}$ and $\mathcal{L}^{*}$ overlap: $\mathcal{L}^{*} \subset \mathcal{K}^{*}$. 
REMARK 3.1. Our definition of dual control volumes differs from the one proposed in $[13,14]$ or [26]. In [14], they built $\mathcal{K}^{*}$ by joining not only the barycenter $x_{\mathcal{K}}$ associated to the elements of the primal mesh of which $x_{\mathcal{K}^{*}}$ is a vertex but also the midpoint of the edges of which $x_{\mathcal{K}^{*}}$ is a vertex. This construction is usually called the barycentric dual mesh. Barycentric dual cells never overlap.

For all control volumes $\mathcal{K}$ and $\mathcal{L}$, we assume that $\partial \mathcal{K} \cap \partial \mathcal{L}$ is either empty or a common vertex or an edge of the primal mesh denoted by $\sigma=\mathcal{K} \mid \mathcal{L}$. We note by $\mathcal{E}$ the set of such edges. We also note $\sigma^{*}=\mathcal{K}^{*} \mid \mathcal{L}^{*}$ and $\mathcal{E}^{*}$ for the corresponding dual definitions.

Given the primal and dual control volumes, we define the diamond cells $\mathcal{D}_{\sigma, \sigma^{*}}$ being the quadrangles whose diagonals are a primal edge $\sigma=\mathcal{K} \mid \mathcal{L}=\left(x_{\mathcal{K}^{*}}, x_{\mathcal{L}^{*}}\right)$ and a corresponding dual edge $\sigma^{*}=\mathcal{K}^{*} \mid \mathcal{L}^{*}=\left(x_{\mathcal{K}}, x_{\mathcal{L}}\right)$, (see Fig. 3.3). Note that the diamond cells are not necessarily convex. If $\sigma \in \mathcal{E} \cap \partial \bar{\Omega}$, the quadrangle $\mathcal{D}_{\sigma, \sigma^{*}}$ degenerate into a triangle (see Fig. 3.3). The set of the diamond cells is denoted by $\mathfrak{D}$ and we have $\bar{\Omega}=\underset{\mathcal{D} \in \mathfrak{D}}{\cup} \overline{\mathcal{D}}$.
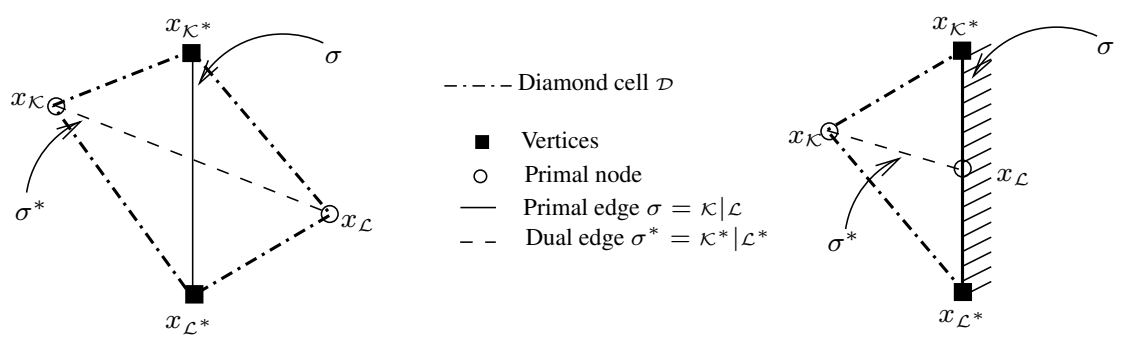

FIG. 3.3. The diamond cells. (Left) Interior diamond cell. (Right) Boundary diamond cell.

Notation. For any primal control volume $\mathcal{K} \in \mathfrak{M} \cup \partial \mathfrak{M}$, we note

- $m_{\mathcal{K}}$ its Lebesgue measure,

- $\mathcal{E}_{\mathcal{K}}$ the set of its edges (if $\mathcal{K} \in \mathfrak{M}$ ), or the one-element set $\{\mathcal{K}\}$ if $\mathcal{K} \in \partial \mathfrak{M}$.

- $\mathfrak{D}_{\mathcal{K}}=\left\{\mathcal{D}_{\sigma, \sigma^{*}} \in \mathfrak{D}, \sigma \in \mathcal{E}_{\mathcal{K}}\right\}$,

- $d_{\mathcal{K}}$ its diameter,

- $\sigma_{\mathcal{K}}:=B\left(x_{\mathcal{K}}, \rho_{\mathcal{K}}\right) \cap \partial \Omega \subset \mathcal{K}$ for $\mathcal{K} \in \partial \mathfrak{M}, m_{\sigma_{\mathcal{K}}}$ its length, the value $\rho_{\mathcal{K}}$ is chosen such that the inclusion is verified.

We will also use corresponding dual notation: $m_{\mathcal{K}^{*}}, \mathcal{E}_{\mathcal{K}^{*}}, \mathfrak{D}_{\mathcal{K}^{*}}, d_{\mathcal{K}^{*}}, \sigma_{\mathcal{K}^{*}}, \rho_{\mathcal{K}^{*}}$ and $m_{\sigma_{\mathcal{K}^{*}}}$.
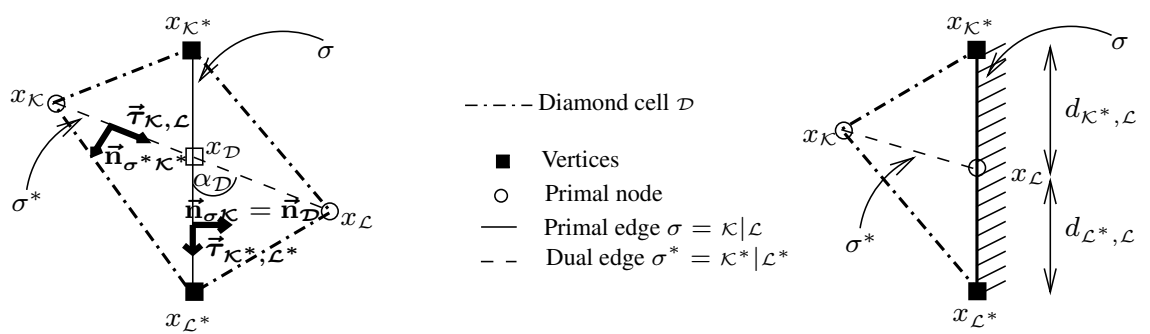

FIG. 3.4. Notations in the diamond cells. (Left) Interior diamond cell. (Right) Boundary diamond cell.

For a diamond cell $\mathcal{D}=\mathcal{D}_{\sigma, \sigma^{*}}$ whose vertices are $\left(x_{\mathcal{K}}, x_{\mathcal{K}^{*}}, x_{\mathcal{L}}, x_{\mathcal{L}^{*}}\right)$ (Fig. 3.4), we note

- $x_{\mathcal{D}}$ the center of the diamond cell $\mathcal{D}: x_{\mathcal{D}}=\sigma \cap \sigma^{*}$,

- $m_{\sigma}$ the length of the primal edge $\sigma$,

- $m_{\sigma^{*}}$ the length of the dual edge $\sigma^{*}$,

- $\overrightarrow{\mathbf{n}}_{\sigma \mathcal{K}}$ the unit vector normal to $\sigma$ oriented from $x_{\mathcal{K}}$ to $x_{\mathcal{L}}$, also noted $\overrightarrow{\mathbf{n}}_{\mathcal{D}}$, 
- $\overrightarrow{\mathbf{n}}_{\sigma^{*} \mathcal{K}^{*}}$ the unit vector normal to $\sigma^{*}$ oriented from $x_{\mathcal{K}^{*}}$ to $x_{\mathcal{L}^{*}}$,

- $\vec{\tau}_{\mathcal{K}, \mathcal{L}}$ the unit vector parallel to $\sigma^{*}$ oriented from $x_{\mathcal{K}}$ to $x_{\mathcal{L}}$,

- $\vec{\tau}_{\mathcal{K}^{*}, \mathcal{L}^{*}}$ the unit vector parallel to $\sigma$ oriented from $x_{\mathcal{K}^{*}}$ to $x_{\mathcal{L}^{*}}$,

- $\alpha_{\mathcal{D}}$ the angle between $\vec{\tau}_{\mathcal{K}, \mathcal{L}}$ and $\vec{\tau}_{\mathcal{K}^{*}, \mathcal{L}^{*}}$,

- $d_{\mathcal{K}^{*}, \mathcal{L}}\left(\right.$ respectively $\left.d_{\mathcal{L}^{*}, \mathcal{L}}\right)$ the length between $x_{\mathcal{K}^{*}}\left(\right.$ respectively $\left.x_{\mathcal{L}^{*}}\right)$ and $x_{\mathcal{L}}$,

- $h_{\mathcal{D}}$ its diameter,

- $\mathfrak{s}$ its edges (for example $\mathfrak{s}=\left[x_{\mathcal{K}}, x_{\mathcal{K}^{*}}\right]$ ),

- $\mathcal{E}_{\mathcal{D}}=\{\mathfrak{s}, \mathfrak{s} \in \partial \mathcal{D}$ and $\mathfrak{s} \not \subset \partial \Omega\}$ the set of interior edges of $\mathcal{D}$,

- $\mathfrak{S}=\left\{\mathfrak{s} \in \mathcal{E}_{\mathcal{D}}, \quad \forall \mathcal{D} \in \mathfrak{D}\right\}$ the set of interior edges of all diamond cells $\mathcal{D} \in \mathfrak{D}$,

- $m_{\mathfrak{s}}$ the length of a diamond edge $\mathfrak{s}$,

- $\overrightarrow{\mathbf{n}}_{\mathfrak{s} \mathcal{D}}$ the unit vector normal to $\mathfrak{s}=\mathcal{D} \mid \mathcal{D}^{\prime}$ oriented from $\mathcal{D}$ to $\mathcal{D}^{\prime}$,

- $m_{\mathcal{D}}$ its measure, which is equal to

$$
m_{\mathcal{D}}=\frac{1}{2} \sin \left(\alpha_{\mathcal{D}}\right) m_{\sigma} m_{\sigma^{*}}
$$

In a diamond cell $\mathcal{D} \in \mathfrak{D}$, we have two direct orthonormal basis: $\left(\overrightarrow{\boldsymbol{\tau}}_{\mathcal{K}^{*}, \mathcal{L}^{*}}, \overrightarrow{\mathbf{n}}_{\sigma \mathcal{K}}\right)$ and $\left(\overrightarrow{\mathbf{n}}_{\sigma^{*} \mathcal{K}^{*}}, \vec{\tau}_{\mathcal{K}, \mathcal{L}}\right)$. We denote by $\overrightarrow{\mathbf{n}}^{\mathcal{D}}=\left(\overrightarrow{\mathbf{n}}_{\mathcal{D}}\right)_{\mathcal{D} \in \mathfrak{D}} \in\left(\mathbb{R}^{2}\right)^{\mathfrak{D}}$. We distinguish the interior diamond cells and the boundary diamond cells:

$$
\mathfrak{D}_{\text {ext }}=\{\mathcal{D} \in \mathfrak{D}, \mathcal{D} \cap \partial \Omega \neq \emptyset\} \quad \text { and } \quad \mathfrak{D}_{\text {int }}=\mathfrak{D} \backslash \mathfrak{D}_{\text {ext }} .
$$

ASSUMPTION 3.1. We assume that all the diamond cells $\mathcal{D}$ are convex.

Assumption 3.1 implies that the center $x_{\mathcal{D}}$ of the diamond cell $\mathcal{D}$ (resp. the node $x_{\mathcal{K}^{*}}$ of the dual cell $\left.\mathcal{K}^{*}\right)$ is inside $\mathcal{D}$ (resp. $\left.\mathcal{K}^{*}\right)$. We also have for all $\left(\mathcal{K}^{*}, \mathcal{L}^{*}\right) \in \mathfrak{M}^{*} \cup \partial \mathfrak{M}^{*}$ such that $\mathcal{K}^{*} \neq \mathcal{L}^{*}$, we have $\stackrel{\circ}{\mathcal{K}^{*}} \cap \frac{\circ}{\mathcal{L}^{*}}=\emptyset$. It is not the case if we do not assume 3.1 (see Figure 3.2).

REMARK 3.2. If Assumption 3.1 is not satisfied, for instance in Figure 3.5, we take the barycentric dual mesh (defined in Remark 3.1). In that case, the center $x_{\mathcal{D}}$ of the diamond cell $\mathcal{D}_{\sigma, \sigma^{*}}$ is defined as the barycenter of the primal edge $\sigma$.

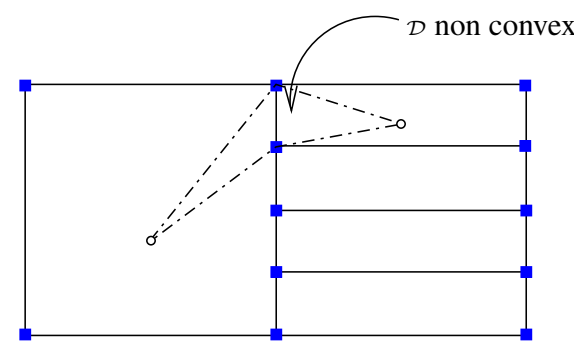

FIG. 3.5. An example where the diamond cells $\mathcal{D}$ could be non convex.

Mesh regularity measurement. Let $\operatorname{size}(\mathcal{T})$ be the maximum of the diameters of the diamond cells in $\mathfrak{D}$. To measure how flat the diamond cells are, we note $\alpha_{\mathcal{T}}$ the unique real in $\left.] 0, \frac{\pi}{2}\right]$ such that $\sin \left(\alpha_{\mathcal{T}}\right):=\min _{\mathcal{D} \in \mathfrak{D}}\left|\sin \left(\alpha_{\mathcal{D}}\right)\right|$. We introduce a positive number $\operatorname{reg}(\mathcal{T})$ that quantifies the regularity of a given mesh and is useful to perform the convergence analysis of finite volume schemes:

$$
\begin{array}{r}
\operatorname{reg}(\mathcal{T}):=\max \left(\frac{1}{\sin \left(\alpha_{\mathcal{T}}\right)}, \mathcal{N}, \mathcal{N}^{*}, \max _{\mathcal{D} \in \mathfrak{D}} \max _{\mathfrak{s} \in \mathcal{E}_{\mathcal{D}}} \frac{h_{\mathcal{D}}}{m_{\mathfrak{s}}}, \max _{\substack{\mathcal{K} \in \mathfrak{M} \\
\mathcal{D} \in \mathfrak{D}_{\mathcal{K}}}} \frac{d_{\mathcal{K}}}{h_{\mathcal{D}}}, \max _{\mathcal{K}^{*} \in \mathfrak{M}^{*} \cup \partial \mathfrak{M}^{*}} \frac{d_{\mathcal{K}^{*}}}{h_{\mathcal{D}}},\right. \\
\left.\max _{\mathcal{D} \in \mathfrak{D} \mathcal{K}^{*}} \frac{h_{\mathcal{D}}}{\sqrt{m_{\mathcal{D}}}}, \max _{\mathcal{K}^{*} \in \mathfrak{M}^{*} \cup \partial \mathfrak{M}^{*}} \frac{d_{\mathcal{K}^{*}}}{\sqrt{m_{\mathcal{K}^{*}}}}, \max _{\mathcal{K} \in \mathfrak{M}} \frac{d_{\mathcal{K}}}{\sqrt{m_{\mathcal{K}}}}\right),
\end{array}
$$


where $\mathcal{N}$ and $\mathcal{N}^{*}$ are the maximum of edges of each primal cell and the maximum of edges incident to any vertex. The number $\operatorname{reg}(\mathcal{T})$ should be uniformly bounded when $\operatorname{size}(\mathcal{T}) \rightarrow 0$ for the convergence to hold.

For instance, this number $\operatorname{reg}(\mathcal{T})$ is involved in the following geometrical result: there exists two constants $C_{1}$ and $C_{2}$ depending on $\operatorname{reg}(\mathcal{T})$ such that for any $\mathcal{K} \in \mathfrak{M}, \mathcal{K}^{*} \in \mathfrak{M}^{*} \cup$ $\partial \mathfrak{M}^{*}$ and $\mathcal{D} \in \mathfrak{D}$ such that $\mathcal{D} \cap \mathcal{K} \neq \emptyset$ and $\mathcal{D} \cap \mathcal{K}^{*} \neq \emptyset$, we have

$$
C_{1} m_{\mathcal{K}} \leq m_{\mathcal{D}} \leq C_{2} m_{\mathcal{K}}, \quad C_{1} m_{\mathcal{K}^{*}} \leq m_{\mathcal{D}} \leq C_{2} m_{\mathcal{K}^{*}} .
$$

3.2. Unknowns and discrete projections. The DDFV method for the Stokes problem requires staggered unknowns. It associates to any primal cell $\mathcal{K} \in \mathfrak{M} \cup \partial \mathfrak{M}$ an unknown value $\mathbf{u}_{\mathcal{K}} \in \mathbb{R}^{2}$ for the velocity, to any dual cell $\mathcal{K}^{*} \in \mathfrak{M}^{*} \cup \partial \mathfrak{M}^{*}$ an unknown value $\mathbf{u}_{\mathcal{K}^{*}} \in \mathbb{R}^{2}$ for the velocity and to any diamond cell $\mathcal{D} \in \mathfrak{D}$ an unknown value $p^{\mathcal{D}} \in \mathbb{R}$ for the pressure. These unknowns are collected in the families :

$$
\begin{aligned}
& \mathbf{u}^{\mathcal{T}}=\left(\left(\mathbf{u}_{\mathcal{K}}\right)_{\mathcal{K} \in(\mathfrak{M} \cup \partial \mathfrak{M})},\left(\mathbf{u}_{\mathcal{K}^{*}}\right)_{\mathcal{K}^{*} \in\left(\mathfrak{M}^{*} \cup \partial \mathfrak{M}^{*}\right)}\right) \in\left(\mathbb{R}^{2}\right)^{\mathcal{T}}, \\
& p^{\mathfrak{D}}=\left(\left(p^{\mathcal{D}}\right)_{\mathcal{D} \in \mathfrak{D}}\right) \in \mathbb{R}^{\mathfrak{D}} .
\end{aligned}
$$

We define now the discrete mean-value boundary data, for any vector function $\mathbf{v}$ lying in $\left(H^{1}(\Omega)\right)^{2}$, denoted by $\mathbb{P}_{m}^{\partial \Omega}$ and defined as follows:

$$
\mathbb{P}_{\boldsymbol{m}}^{\partial \Omega} \mathbf{v}=\left(\left(\frac{1}{m_{\sigma_{\mathcal{K}}}} \int_{\sigma_{\mathcal{K}}} \mathbf{v}(x) \mathrm{d} x\right)_{\mathcal{K} \in \partial \mathfrak{M}},\left(\frac{1}{m_{\sigma_{\mathcal{K}^{*}}}} \int_{\sigma_{\mathcal{K}^{*}}} \mathbf{v}(x) \mathrm{d} x\right)_{\mathcal{K}^{*} \in \partial \mathfrak{M}^{*}}\right),
$$

and the interior mean-value projection for any integrable vector function $\mathbf{v}$ on $\Omega$ :

$$
\mathbb{P}_{\boldsymbol{m}}^{\mathfrak{M} \mathbf{v}}=\left(\left(\frac{1}{m_{\mathcal{K}}} \int_{\mathcal{K}} \mathbf{v}(x) \mathrm{d} x\right)_{\mathcal{K} \in \mathfrak{M}}\right), \mathbb{P}_{\boldsymbol{m}}^{\mathfrak{\mathfrak { M } ^ { * }} \mathbf{v}}=\left(\left(\frac{1}{m_{\mathcal{K}^{*}}} \int_{\mathcal{K}^{*}} \mathbf{v}(x) \mathrm{d} x\right)_{\mathcal{K}^{*} \in \mathfrak{M}^{*}}\right) .
$$

We finally gather these projections in the following notation:

$$
\mathbb{P}_{\boldsymbol{m}}^{\mathcal{T}} \mathbf{v}=\left(\mathbb{P}_{\boldsymbol{m}}^{\mathfrak{m}} \mathbf{v}, \mathbb{P}_{\boldsymbol{m}}^{\mathfrak{M} *} \mathbf{v}, \mathbb{P}_{\boldsymbol{m}}^{\partial \Omega} \mathbf{v}\right), \quad \forall \mathbf{v} \in\left(H^{1}(\Omega)\right)^{2}
$$

We introduce the center-value projection:

$$
\mathbb{P}_{\boldsymbol{c}}^{\mathcal{T}} \mathbf{v}=\left(\left(\mathbf{v}\left(x_{\mathcal{K}}\right)\right)_{\mathcal{K} \in(\mathfrak{M} \cup \partial \mathfrak{M})},\left(\mathbf{v}\left(x_{\mathcal{K}^{*}}\right)\right)_{\mathcal{K}^{*} \in\left(\mathfrak{M}^{*} \cup \partial \mathfrak{M}^{*}\right)}\right), \quad \forall \mathbf{v} \in\left(H^{2}(\Omega)\right)^{2} .
$$

We also define a mean-value projection on $\Omega$ over the diamond mesh $\mathcal{D}$ for any integrable function $q$ :

$$
\mathbb{P}_{m}^{\mathfrak{D}} q=\left(\left(\frac{1}{m_{\mathcal{D}}} \int_{\mathcal{D}} q(x) \mathrm{d} x\right)_{\mathcal{D} \in \mathfrak{D}}\right)
$$

We specify two discrete subsets of $\left(\mathbb{R}^{2}\right)^{T}$ needed to take into account the Dirichlet boundary conditions

$$
\begin{aligned}
& \mathbb{E}_{0}=\left\{\mathbf{v}^{\mathcal{T}} \in\left(\mathbb{R}^{2}\right)^{\mathcal{T}} \text { s. t. } \mathbf{v}_{\mathcal{K}}=0, \forall \mathcal{K} \in \partial \mathfrak{M} \text { and } \mathbf{v}_{\mathcal{K}^{*}}=0, \forall \mathcal{K}^{*} \in \partial \mathfrak{M}^{*}\right\}, \\
& \mathbb{E}_{g}=\left\{\mathbf{v}^{\mathcal{T}} \in\left(\mathbb{R}^{2}\right)^{\mathcal{T}} \text { s. t. } \mathbf{v}_{\mathcal{K}}=\left(\mathbb{P}_{\boldsymbol{m}}^{\partial \Omega} \mathbf{g}\right)_{\mathcal{K}}, \forall \mathcal{K} \in \partial \mathfrak{M} \text { and } \mathbf{v}_{\mathcal{K}^{*}}=\left(\mathbb{P}_{\boldsymbol{m}}^{\partial \Omega} \mathbf{g}\right)_{\mathcal{K}^{*}}, \forall \mathcal{K}^{*} \in \partial \mathfrak{M}^{*}\right\} .
\end{aligned}
$$

We define the projection $\mathfrak{P}_{m, g}$ on the set $\mathbb{E}_{g}$ :

$$
\begin{aligned}
\mathfrak{P}_{\boldsymbol{m}, \boldsymbol{g}}:\left(\mathbb{R}^{2}\right)^{\mathcal{T}} & \longrightarrow \mathbb{E}_{g} \\
\mathbf{u}^{\mathcal{T}} & \longmapsto\left(\left(\mathbf{u}_{\mathcal{K}}\right)_{\mathcal{K} \in \mathfrak{M}},\left(\mathbb{P}_{\boldsymbol{m}}^{\partial \boldsymbol{\Omega}} \mathbf{g}\right)_{\mathcal{K} \in \partial \mathfrak{M}},\left(\mathbf{u}_{\mathcal{K}^{*}}\right)_{\mathcal{K}^{*} \in \mathfrak{M}^{*}},\left(\mathbb{P}_{\boldsymbol{m}}^{\partial \boldsymbol{g}} \mathbf{g}\right)_{\mathcal{K}^{*} \in \partial \mathfrak{M}^{*}}\right) .
\end{aligned}
$$


3.3. Discrete operators. In this subsection, we define the discrete operators which are needed in order to write and analyse the DDFV scheme. We begin with the discrete gradient.

DEFINITION 3.1 (Discrete gradient). We define a consistent approximation of the gradient operator of a vector field in $\left(\mathbb{R}^{2}\right)^{\mathcal{T}}$ denoted by $\nabla^{\mathfrak{D}}: \mathbf{u}^{\mathcal{T}} \in\left(\mathbb{R}^{2}\right)^{\mathcal{T}} \mapsto\left(\nabla^{\mathcal{D}} \mathbf{u}^{\mathcal{T}}\right)_{\mathcal{D} \in \mathfrak{D}} \in$ $\left(\mathcal{M}_{2}(\mathbb{R})\right)^{\mathfrak{D}}$, as follows:

$$
\nabla^{\mathcal{D}} \mathbf{u}^{\mathcal{T}}=\frac{1}{\sin \left(\alpha_{\mathcal{D}}\right)}\left[\frac{\mathbf{u}_{\mathcal{L}}-\mathbf{u}_{\mathcal{K}}}{m_{\sigma^{*}}} \otimes \overrightarrow{\mathbf{n}}_{\sigma \mathcal{K}}+\frac{\mathbf{u}_{\mathcal{L}^{*}}-\mathbf{u}_{\mathcal{K}^{*}}}{m_{\sigma}} \otimes \overrightarrow{\mathbf{n}}_{\sigma^{*} \mathcal{K}^{*}}\right],
$$

where $\otimes$ represents the tensor product. It can also be written as follows, (see (3.1)):

$$
\nabla^{\mathcal{D}} \mathbf{u}^{\mathcal{T}}=\frac{1}{2 m_{\mathcal{D}}}\left[m_{\sigma}\left(\mathbf{u}_{\mathcal{L}}-\mathbf{u}_{\mathcal{K}}\right) \otimes \overrightarrow{\mathbf{n}}_{\sigma \mathcal{K}}+m_{\sigma^{*}}\left(\mathbf{u}_{\mathcal{L}^{*}}-\mathbf{u}_{\mathcal{K}^{*}}\right) \otimes \overrightarrow{\mathbf{n}}_{\sigma^{*} \mathcal{K}^{*}}\right]
$$

REMARK 3.3. We recall the discrete gradient operator for a scalar field in $\mathbb{R}^{\mathcal{T}}$ still denoted by $\nabla^{\mathfrak{D}}: u^{\mathcal{T}} \in \mathbb{R}^{\mathcal{T}} \mapsto\left(\nabla^{\mathcal{D}} u^{\mathcal{T}}\right)_{\mathcal{D} \in \mathfrak{D}} \in\left(\mathbb{R}^{2}\right)^{\mathfrak{D}}$, which is defined as follows:

$$
\nabla^{\mathcal{D}} u^{\mathcal{T}}=\frac{1}{2 m_{\mathcal{D}}}\left[\left(u_{\mathcal{L}}-u_{\mathcal{K}}\right) m_{\sigma} \overrightarrow{\mathbf{n}}_{\sigma \mathcal{K}}+\left(u_{\mathcal{L}^{*}}-u_{\mathcal{K}^{*}}\right) m_{\sigma^{*}} \overrightarrow{\mathbf{n}}_{\sigma^{*}} \mathcal{K}^{*}\right], \quad \forall \mathcal{D} \in \mathfrak{D} .
$$

REMARK 3.4. For two neighbour cells $\mathcal{K}$ and $\mathcal{L}$ sharing a face $F$, we can naturally define two directions: the line $x_{\mathcal{K}} x_{\mathcal{L}}$ and one edge $e \subset \partial F$. The difference in the $3 D$ approach comes from the choice of the third complementary direction. In $[1,11]$ this third direction is given by a different edge $e^{\prime} \subset \partial F$ of the face $F$, restrictions on the primal mesh are needed. In [27, 9], the third direction is given by $x_{F} x_{e}$ where $x_{F}$ and $x_{e}$ are the barycenter of the face $F$ and the edge $e \subset \partial F$.

DEFINITION 3.2 (Discrete divergence). We define a consistent approximation of the divergence operator applied to discrete tensor fields denoted by $\operatorname{div}^{\mathcal{T}}: \xi^{\mathfrak{D}}=\left(\xi^{\mathcal{D}}\right)_{\mathcal{D} \in \mathfrak{D}} \in$ $\left(\mathcal{M}_{2}(\mathbb{R})\right)^{\mathfrak{D}} \mapsto \operatorname{div}^{\mathcal{T}} \xi^{\mathfrak{D}} \in\left(\mathbb{R}^{2}\right)^{\mathcal{T}}$, as follows:

$$
\begin{aligned}
\operatorname{div}^{\mathcal{K}} \xi^{\mathfrak{D}} & =\frac{1}{m_{\mathcal{K}}} \sum_{\sigma \in \partial \mathcal{K}} m_{\sigma} \xi^{\mathcal{D}} \overrightarrow{\mathbf{n}}_{\sigma \mathcal{K}}, \forall \mathcal{K} \in \mathfrak{M}, \text { and } \operatorname{div}^{\mathcal{K}} \xi^{\mathfrak{D}}=0, \forall \mathcal{K} \in \partial \mathfrak{M}, \\
\operatorname{div}^{\mathcal{K}^{*}} \xi^{\mathfrak{D}} & =\frac{1}{m_{\mathcal{K}^{*}}} \sum_{\sigma^{*} \in \partial \mathcal{K}^{*}} m_{\sigma^{*}} \xi^{\mathcal{D}} \overrightarrow{\mathbf{n}}_{\sigma^{*} \mathcal{K}^{*}}, \forall \mathcal{K}^{*} \in \mathfrak{M}^{*}, \\
\operatorname{div}^{\mathcal{K}^{*}} \xi^{\mathfrak{D}} & =\frac{1}{m_{\mathcal{K}^{*}}}\left(\sum_{\mathcal{D}_{\sigma, \sigma^{*}} \in \mathfrak{D}_{\mathcal{K}^{*}}} m_{\sigma^{*}} \xi^{\mathcal{D}} \overrightarrow{\mathbf{n}}_{\sigma^{*}} \mathcal{K}^{*}+\sum_{\mathcal{D}_{\sigma, \sigma^{*}} \in \mathfrak{D}_{\mathcal{K}^{*} \cap \mathfrak{D}_{e x t}}} d_{\mathcal{K}^{*}, \mathcal{L}} \xi^{\mathcal{D}} \overrightarrow{\mathbf{n}}_{\sigma \mathcal{K}}\right), \forall \mathcal{K}^{*} \in \partial \mathfrak{M}^{*} .
\end{aligned}
$$

In order to write the DDFV scheme in a compact form, we will denote the discrete divergence on the primal mesh and on the interior dual mesh as follows:

$$
\operatorname{div}^{\mathfrak{M}} \xi^{\mathfrak{D}}=\left(\left(\operatorname{div}^{\mathcal{K}} \xi^{\mathfrak{D}}\right)_{\mathcal{K} \in \mathfrak{M}}\right), \quad \operatorname{div}^{\mathfrak{M}^{*}} \xi^{\mathfrak{D}}=\left(\left(\operatorname{div}^{\mathcal{K}^{*}} \xi^{\mathfrak{D}}\right)_{\mathcal{K}^{*} \in \mathfrak{M}^{*}}\right)
$$

Thanks to the discrete gradient we can define a discrete strain rate tensor and a discrete divergence of a vector field in $\left(\mathbb{R}^{2}\right)^{\mathcal{T}}$.

DEFINITION 3.3 (Discrete strain rate tensor). We define a discrete strain rate tensor of a vector field in $\left(\mathbb{R}^{2}\right)^{\mathcal{T}}$, denoted by: $\mathrm{D}^{\mathfrak{D}}: \mathbf{u}^{\mathcal{T}} \in\left(\mathbb{R}^{2}\right)^{\mathcal{T}} \mapsto\left(\mathrm{D}^{\mathcal{D}} \mathbf{u}^{\mathcal{T}}\right)_{\mathcal{D} \in \mathfrak{D}} \in\left(\mathcal{M}_{2}(\mathbb{R})\right)^{\mathfrak{D}}$, with $\mathrm{D}^{\mathcal{D}} \mathbf{u}^{\mathcal{T}}=\frac{\nabla^{\mathcal{D}} \mathbf{u}^{\mathcal{T}}+{ }^{t}\left(\nabla^{\mathcal{D}} \mathbf{u}^{\mathcal{T}}\right)}{2}$, for all $\mathcal{D} \in \mathfrak{D}$. 
DEFINITION 3.4. We define a discrete divergence of a vector field in $\left(\mathbb{R}^{2}\right)^{\mathcal{T}}$, denoted by: $\operatorname{div}^{\mathcal{D}}: \mathbf{u}^{\mathcal{T}} \in\left(\mathbb{R}^{2}\right)^{\mathcal{T}} \mapsto\left(\operatorname{div}^{\mathcal{D}} \mathbf{u}^{\mathcal{T}}\right)_{\mathcal{D} \in \mathfrak{D}} \in \mathbb{R}^{\mathfrak{D}}$, with $\operatorname{div}^{\mathcal{D}} \mathbf{u}^{\mathcal{T}}=\operatorname{Trace}\left(\nabla^{\mathcal{D}} \mathbf{u}^{\mathcal{T}}\right)$, for all $\mathcal{D} \in \mathfrak{D}$.

REMARK 3.5. Remark that $\operatorname{div}^{\mathcal{D}} \mathbf{u}^{\mathcal{T}}$ can be expressed in the following way:

$$
\operatorname{div}^{\mathcal{D}} \mathbf{u}^{\mathcal{T}}=\frac{1}{2 m_{\mathcal{D}}}\left[m_{\sigma}\left(\mathbf{u}_{\mathcal{L}}-\mathbf{u}_{\mathcal{K}}\right) \cdot \overrightarrow{\mathbf{n}}_{\sigma \mathcal{K}}+m_{\sigma^{*}}\left(\mathbf{u}_{\mathcal{L}^{*}}-\mathbf{u}_{\mathcal{K}^{*}}\right) \cdot \overrightarrow{\mathbf{n}}_{\sigma^{*} \mathcal{K}^{*}}\right]
$$

and

$$
\operatorname{div}^{\mathcal{D}}\left(\mathbf{u}^{\mathcal{T}}\right)=\frac{1}{m_{\mathcal{D}}} \sum_{\mathfrak{s}=\left[x_{\mathcal{K}}, x_{\mathcal{K}^{*}}\right] \in \mathcal{E}_{\mathcal{D}}} m_{\mathfrak{s}} \frac{\mathbf{u}_{\mathcal{K}^{*}}+\mathbf{u}_{\mathcal{K}}}{2} \cdot \overrightarrow{\mathbf{n}}_{\mathfrak{s} \mathcal{D}}, \quad \forall \mathbf{u}^{\mathcal{T}} \in\left(\mathbb{R}^{2}\right)^{\mathcal{T}}
$$

The relation 3.9 is the discrete counterpart of $\int_{\mathcal{D}} \operatorname{div}(\mathbf{u})(z) \mathrm{d} z=\int_{\mathcal{E}_{\mathcal{D}}} \mathbf{u}(s) \cdot \overrightarrow{\mathbf{n}}_{\mathcal{D}} \mathrm{d} s$.

3.4. Inner products and norms. First of all, we define trace operators on both $\left(\mathbb{R}^{2}\right)^{\mathcal{T}}$ and $\left(\mathbb{R}^{2}\right)^{\mathfrak{D}}$. Set $\boldsymbol{\gamma}^{\mathcal{T}}: \mathbf{u}^{\mathcal{T}} \in\left(\mathbb{R}^{2}\right)^{\mathcal{T}} \mapsto \boldsymbol{\gamma}^{\mathcal{T}}\left(\mathbf{u}^{\mathcal{T}}\right)=\left(\boldsymbol{\gamma}_{\boldsymbol{\sigma}}\left(\mathbf{u}^{\mathcal{T}}\right)\right)_{\sigma \in \partial \mathfrak{M}} \in\left(\mathbb{R}^{2}\right)^{\partial \mathfrak{M}}$, as follows:

$$
\gamma_{\boldsymbol{\sigma}}\left(\mathbf{u}^{\mathcal{T}}\right)=\frac{d_{\mathcal{K}^{*}, \mathcal{L}}\left(\mathbf{u}_{\mathcal{K}^{*}}+\mathbf{u}_{\mathcal{L}}\right)+d_{\mathcal{L}^{*}, \mathcal{L}}\left(\mathbf{u}_{\mathcal{L}^{*}}+\mathbf{u}_{\mathcal{L}}\right)}{2 m_{\sigma}}, \quad \forall \sigma \in \partial \mathfrak{M} .
$$

This trace operator enables to impose the Dirichlet boundary conditions in a weak way. The second one is denoted by $\gamma^{\mathfrak{D}}: \phi^{\mathfrak{D}} \in\left(\mathbb{R}^{2}\right)^{\mathfrak{D}} \mapsto\left(\phi^{\mathcal{D}}\right)_{\mathcal{D} \in \mathfrak{D}_{\text {ext }}} \in\left(\mathbb{R}^{2}\right)^{\mathfrak{D}_{\text {ext }}}$ and is only a restriction operator on $\mathfrak{D}_{\text {ext }}$. Then, we define the four following inner products

$$
\begin{aligned}
\llbracket \mathbf{v}^{\mathcal{T}}, \mathbf{u}^{\mathcal{T}} \rrbracket_{\mathcal{T}} & =\frac{1}{2}\left(\sum_{\mathcal{K} \in \mathfrak{M}} m_{\mathcal{K}} \mathbf{u}_{\mathcal{K}} \cdot \mathbf{v}_{\mathcal{K}}+\sum_{\mathcal{K}^{*} \in \mathfrak{M}^{*} \cup \partial \mathfrak{M}^{*}} m_{\mathcal{K}^{*}} \mathbf{u}_{\mathcal{K}^{*}} \cdot \mathbf{v}_{\mathcal{K}^{*}}\right), \quad \forall \mathbf{u}^{\mathcal{T}}, \mathbf{v}^{\mathcal{T}} \in\left(\mathbb{R}^{2}\right)^{\mathcal{T}}, \\
\left(\boldsymbol{\phi}^{\mathfrak{D}}, \mathbf{v}^{\mathcal{T}}\right)_{\partial \Omega} & =\sum_{\mathcal{D}_{\sigma, \sigma} \in \mathfrak{D}_{e x t}} m_{\sigma} \boldsymbol{\phi}^{\mathcal{D}} \cdot \mathbf{v}_{\boldsymbol{\sigma}}, \quad \forall \boldsymbol{\phi}^{\mathfrak{D}} \in\left(\mathbb{R}^{2}\right)^{\mathfrak{D}_{e x t}}, \mathbf{v}^{\mathcal{T}} \in\left(\mathbb{R}^{2}\right)^{\partial \mathfrak{M}}, \\
\left(p^{\mathfrak{D}}, q^{\mathfrak{D}}\right)_{\mathfrak{D}} & =\sum_{\mathcal{D} \in \mathfrak{D}} m_{\mathcal{D}} p^{\mathcal{D}} q^{\mathcal{D}}, \quad \forall p^{\mathfrak{D}}, q^{\mathfrak{D}} \in \mathbb{R}^{\mathfrak{D}}, \\
\left(\xi^{\mathfrak{D}}: \phi^{\mathfrak{D}}\right)_{\mathfrak{D}} & =\sum_{\mathcal{D} \in \mathfrak{D}} m_{\mathcal{D}}\left(\xi^{\mathcal{D}}: \phi^{\mathcal{D}}\right), \quad \forall \xi^{\mathfrak{D}}, \phi^{\mathfrak{D}} \in\left(\mathcal{M}_{2}(\mathbb{R})\right)^{\mathfrak{D}},
\end{aligned}
$$

(recall that $\left.(\xi: \widetilde{\xi})=\operatorname{Trace}\left({ }^{t} \xi \widetilde{\xi}\right)\right)$. We define the corresponding norms as follows

$$
\begin{array}{rlrl}
\left\|\mathbf{u}^{\mathcal{T}}\right\|_{2} & =\llbracket \mathbf{u}^{\mathcal{T}}, \mathbf{u}^{\mathcal{T}} \rrbracket_{\mathcal{T}}^{\frac{1}{2}}, & & \forall \mathbf{u}^{\mathcal{T}} \in\left(\mathbb{R}^{2}\right)^{\mathcal{T}}, \\
\left\|p^{\mathfrak{D}}\right\|_{2}=\left(p^{\mathfrak{D}}, p^{\mathfrak{D}}\right)_{\mathfrak{D}}^{\frac{1}{2}}, & \forall p^{\mathfrak{D}} \in \mathbb{R}^{\mathfrak{D}}, \\
\left\|\xi^{\mathfrak{D}}\right\|_{2}=\left(\xi^{\mathfrak{D}}: \xi^{\mathfrak{D}}\right)_{\mathfrak{D}}^{\frac{1}{2}}, & \forall \xi^{\mathfrak{D}} \in\left(\mathcal{M}_{2}(\mathbb{R})\right)^{\mathfrak{D}} .
\end{array}
$$

3.5. Discrete Stokes formula. In $[2,14,15]$, the discrete gradient and discrete divergence for a scalar-value function are linked by a discrete Stokes formula, which is the duality property giving its name to the method. We want to generalize the discrete Stokes formula for vector-valued functions. The discrete Stokes formula for vector-valued functions is deduced from its scalar counterpart (given in [2]) by working component per component.

THEOREM 3.1 (Discrete Stokes formula). For all $\xi^{\mathfrak{D}} \in\left(\mathcal{M}_{2}(\mathbb{R})\right)^{\mathfrak{D}}, \mathbf{u}^{\mathcal{T}} \in\left(\mathbb{R}^{2}\right)^{\mathcal{T}}$ :

$$
\llbracket \operatorname{div}^{\mathcal{T}} \xi^{\mathfrak{D}}, \mathbf{u}^{\mathcal{T}} \rrbracket_{\mathcal{T}}=-\left(\xi^{\mathfrak{D}}: \nabla^{\mathfrak{D}} \mathbf{u}^{\mathcal{T}}\right)_{\mathfrak{D}}+\left(\gamma^{\mathfrak{D}}\left(\xi^{\mathfrak{D}} \overrightarrow{\mathbf{n}}^{\mathcal{D}}\right), \gamma^{\mathcal{T}}\left(\mathbf{u}^{\mathcal{T}}\right)\right)_{\partial \Omega}
$$


3.6. Preparation of the stabilization procedure. Finally, a second order discrete difference operator will be needed to define the stabilization term in the Stokes problem.

DEFINITION 3.5. We define a non consistent discrete approximation of the laplacian $\Delta p$, denoted by $\Delta^{\mathfrak{D}}: p^{\mathfrak{D}} \in \mathbb{R}^{\mathfrak{D}} \mapsto \Delta^{\mathfrak{D}} p^{\mathfrak{D}} \in \mathbb{R}^{\mathfrak{D}}$, and defined as follows:

$$
\Delta^{\mathcal{D}} p^{\mathfrak{D}}=\frac{1}{m_{\mathcal{D}}} \sum_{\mathfrak{s}=\mathcal{D} \mid \mathcal{D}^{\prime} \in \mathcal{E}_{\mathcal{D}}} \frac{h_{\mathcal{D}}^{2}+h_{\mathcal{D}^{\prime}}^{2}}{h_{\mathcal{D}}^{2}}\left(p^{\mathcal{D}^{\prime}}-p^{\mathcal{D}}\right), \quad \forall \mathcal{D} \in \mathfrak{D} .
$$

Note that we do not need a consistent approximation of the Laplace operator. In fact, a consistent approximation based on a two-point flux formula would require the diamond mesh to verify an orthogonality constraint as, for instance, in the case of admissible meshes [18], which has no reason to hold here.

Related to this operator, we define a mesh dependent semi-norm $|\cdot|_{h}$ over $\mathbb{R}^{\mathfrak{D}}$ by:

$$
\left|p^{\mathfrak{D}}\right|_{h}^{2}=\sum_{\mathfrak{s}=\mathcal{D} \mid \mathcal{D}^{\prime} \in \mathfrak{S}}\left(h_{\mathcal{D}}^{2}+h_{\mathcal{D}^{\prime}}^{2}\right)\left(p^{\mathcal{D}^{\prime}}-p^{\mathcal{D}}\right)^{2}, \quad \forall p^{\mathfrak{D}} \in \mathbb{R}^{\mathfrak{D}} .
$$

The semi-norm $|p|_{h}$ is the discrete counterpart of $\operatorname{size}(\mathcal{T})|\nabla p|_{2}$.

REMARK 3.6. We have, for any $p^{\mathfrak{D}} \in \mathbb{R}^{\mathfrak{D}}$, by reorganizing the summation over $\mathfrak{s} \in \mathfrak{S}$,

$$
\begin{aligned}
-\left(h_{\mathfrak{D}}^{2} \Delta^{\mathfrak{D}} p^{\mathfrak{D}}, p^{\mathfrak{D}}\right)_{\mathfrak{D}} & =\sum_{\mathcal{D} \in \mathfrak{D}} p^{\mathcal{D}} \sum_{\mathfrak{s} \in \mathcal{E}_{\mathcal{D}}}\left(h_{\mathcal{D}}^{2}+h_{\mathcal{D}^{\prime}}^{2}\right)\left(p^{\mathcal{D}}-p^{\mathcal{D}^{\prime}}\right) \\
& =\sum_{\mathfrak{s}=\mathcal{D} \mid \mathcal{D}^{\prime} \in \mathfrak{S}}\left(h_{\mathcal{D}}^{2}+h_{\mathcal{D}^{\prime}}^{2}\right)\left(p^{\mathcal{D}^{\prime}}-p^{\mathcal{D}}\right)^{2} \\
& =\left|p^{\mathfrak{D}}\right|_{h}^{2} .
\end{aligned}
$$

The following Lemma is an inverse Sobolev lemma, that is a bound of the discrete seminorm $|\cdot|_{h}$, defined by (3.10), by the $L^{2}$-norm $\|\cdot\|_{2}$.

LEMMA 3.1. Let $\mathcal{T}$ be a mesh of $\Omega$. There exists $C>0$ depending only on $\operatorname{reg}(\mathcal{T})$, such that for any $p^{\mathfrak{D}} \in \mathbb{R}^{\mathfrak{D}}$, we have

$$
\left|p^{\mathfrak{D}}\right|_{h} \leq C\left\|p^{\mathfrak{D}}\right\|_{2}
$$

Proof. Using the definition (3.10) of the discrete semi-norm $|\cdot|_{h}$ and the Young inequality, we have

$$
\left|p^{\mathfrak{D}}\right|_{h}^{2} \leq 2 \sum_{\mathfrak{s}=\mathcal{D} \mid \mathcal{D}^{\prime} \in \mathfrak{S}}\left(h_{\mathcal{D}}^{2}+h_{\mathcal{D}^{\prime}}^{2}\right)\left(\left(p^{\mathcal{D}^{\prime}}\right)^{2}+\left(p^{\mathcal{D}}\right)^{2}\right) .
$$

Reordering the summation over the set of diamond cells, we get

$$
\left|p^{\mathfrak{D}}\right|_{h}^{2} \leq 2 \sum_{\mathcal{D} \in \mathfrak{D}} m_{\mathcal{D}}\left(p^{\mathcal{D}}\right)^{2}\left(\frac{1}{m_{\mathcal{D}}} \sum_{\mathfrak{s}=\mathcal{D} \mid \mathcal{D}^{\prime} \in \mathcal{E}_{\mathcal{D}}}\left(h_{\mathcal{D}}^{2}+h_{\mathcal{D}^{\prime}}^{2}\right)\right) .
$$

We conclude, using the relation (3.2) and the fact that $\#\left(\mathcal{E}_{\mathcal{D}}\right) \leq 4$.

4. DDFV schemes for the Stokes equation. We note $\eta_{\mathcal{D}}=\int_{\overline{\mathcal{D}}} \eta(s) \mathrm{d} \mu_{\overline{\mathcal{D}}}(s)$, for all $\mathcal{D} \in \mathfrak{D}$, where $\mu_{\overline{\mathcal{D}}}$ is a probability measure on $\overline{\mathcal{D}}$. This includes the case $\eta_{\mathcal{D}}=\eta\left(x_{\mathcal{D}}\right)$ or $\eta_{\mathcal{D}}=\frac{1}{m_{\mathcal{D}}} \int_{\mathcal{D}} \eta(x) \mathrm{d} x$. Furthermore, we always have the following inequality

$$
\underline{\mathrm{C}}_{\eta} \leq \eta_{\mathcal{D}} \leq \overline{\mathrm{C}}_{\eta}, \forall \mathcal{D} \in \mathfrak{D}
$$


As claimed in introduction, we approximate the velocity on both vertices and centers of primal control volumes and the pressure on the diamond cells. We integrate the momentum conservation law of problem (2.1) on the primal mesh $\mathfrak{M}$ and on the interior dual mesh $\mathfrak{M}^{*}$. The mass conservation equation is directly approached on the diamond mesh using the discrete operator $\operatorname{div}^{\mathfrak{D}}$ and a stabilized term inspired by the well known Brezzi-Pitkäranta scheme. We impose the Dirichlet boundary conditions on $\partial \mathfrak{M}$ and on $\partial \mathfrak{M}^{*}$. Finally, the integral of the pressure is imposed to be equal to zero.

The scheme for the problem (2.1) reads as follows:

$$
\left\{\begin{array}{l}
\text { Find } \mathbf{u}^{\mathcal{T}} \in \mathbb{E}_{g} \text { and } p^{\mathfrak{D}} \in \mathbb{R}^{\mathfrak{D}} \text { such that } \\
\operatorname{div}^{\mathfrak{M}}\left(-2 \eta^{\mathfrak{D}} \mathrm{D}^{\mathfrak{D}} \mathbf{u}^{\mathcal{T}}+p^{\mathfrak{D}} \mathrm{Id}\right)=\mathbf{f}^{\mathfrak{M}} \\
\operatorname{div}^{\mathfrak{M}}\left(-2 \eta^{\mathfrak{D}} \mathrm{D}^{\mathfrak{D}} \mathbf{u}^{\mathcal{T}}+p^{\mathfrak{D}} \mathrm{Id}\right)=\mathbf{f}^{\mathfrak{M} *} \\
\operatorname{div}^{\mathfrak{D}}\left(\mathbf{u}^{\mathcal{T}}\right)-\lambda h_{\mathfrak{D}}^{2} \Delta^{\mathfrak{D}} p^{\mathfrak{D}}=0 \\
\sum_{\mathcal{D} \in \mathfrak{D}} m_{\mathcal{D}} p^{\mathcal{D}}=0
\end{array}\right.
$$

with $\lambda>0$ given, $\mathbf{f}^{\mathfrak{M}}=\mathbb{P}_{\boldsymbol{m}}^{\mathfrak{M}} \mathbf{f}$ and $\mathbf{f}^{\mathfrak{M}^{*}}=\mathbb{P}_{\boldsymbol{m}}^{\mathfrak{M}^{*}} \mathbf{f}$, where the projection is defined by (3.4).

REMARK 4.1. At the continuous level, we have a compatibility condition (2.2). The same relation still holds at the discrete level. Indeed, we have by using the discrete Stokes formula Theorem 3.1

$$
\begin{aligned}
\left(\operatorname{div}^{\mathfrak{D}}\left(\mathbf{u}^{\mathcal{T}}\right)-\lambda h_{\mathfrak{D}}^{2} \Delta^{\mathfrak{D}} p^{\mathfrak{D}}, 1\right)_{\mathfrak{D}} & =\left(\operatorname{div}^{\mathfrak{D}} \mathbf{u}^{\mathcal{T}}, 1\right)_{\mathfrak{D}}=\left(\nabla^{\mathfrak{D}} \mathbf{u}^{\mathcal{T}}: \operatorname{Id}\right)_{\mathfrak{D}} \\
& =\left(\boldsymbol{\gamma}^{\mathfrak{D}}\left(\overrightarrow{\mathbf{n}}^{\mathfrak{D}}\right), \boldsymbol{\gamma}^{\mathcal{T}}\left(\mathbf{u}^{\mathcal{T}}\right)\right)_{\partial \Omega}
\end{aligned}
$$

Using the scheme (4.1), we obtain the following condition

$$
\sum_{\mathcal{D}_{\sigma, \sigma^{*}} \in \mathfrak{D}_{e x t}} m_{\sigma} \gamma_{\boldsymbol{\sigma}}\left(\mathbf{g}^{\mathcal{T}}\right) \cdot \overrightarrow{\mathbf{n}}_{\sigma \mathcal{K}}=0 .
$$

We deduce a link between the mass conservation equation and the Dirichlet boundary condition.

REMARK 4.2. In (4.1), we have added the discrete counterpart of $\int_{\Omega} p(x) \mathrm{d} x=0$, that is $\sum_{\mathcal{D} \in \mathfrak{D}} m_{\mathcal{D}} p^{\mathcal{D}}=0$ in order to ensure the uniqueness of the pressure.

REMARK 4.3. Note that we impose the Dirichlet boundary conditions on $\partial \mathfrak{M}^{*}$, so that we do not integrate the momentum conservation law of problem (2.1) on $\partial \mathfrak{M}^{*}$. An alternative scheme would be for instance

$$
\left\{\begin{array}{l}
\text { Find } \mathbf{u}^{\mathcal{T}} \in\left(\mathbb{R}^{2}\right)^{\mathcal{T}} \text { and } p^{\mathfrak{D}} \in \mathbb{R}^{\mathfrak{D}} \text { such that } \\
\operatorname{div}^{\mathcal{T}}\left(-2 \eta^{\mathfrak{D}} \mathrm{D}^{\mathfrak{D}} \mathbf{u}^{\mathcal{T}}+p^{\mathfrak{D}} \mathrm{Id}\right)=\mathbf{f}^{\mathcal{T}} \\
\gamma^{\mathcal{T}}\left(\mathbf{u}^{\mathcal{T}}\right)=\mathbf{g}^{\partial \mathfrak{M}} \\
\operatorname{div}^{\mathfrak{D}}\left(\mathbf{u}^{\mathcal{T}}\right)-\lambda h_{\mathfrak{D}}^{2} \Delta^{\mathfrak{D}} p^{\mathfrak{D}}=0 \\
\sum_{\mathcal{D} \in \mathfrak{D}} m_{\mathcal{D}} p^{\mathcal{D}}=0
\end{array}\right.
$$

Since even for $\mathbf{g}=0$ we are not sure that $\mathbf{u}^{\boldsymbol{T}} \in \mathbb{E}_{0}$, the well-posedness and the stability of this scheme is still an open problem (it is not known if Korn inequality holds in this case, see Theorem 5.1).

THEOREM 4.1. Assume that $\eta$ satisfies (2.3). For any mesh $\mathcal{T}$ as described in Section 3, the finite volume scheme (4.1) with $\lambda>0$ admits a unique solution $\left(\mathbf{u}^{\mathcal{T}}, p^{\mathfrak{D}}\right) \in\left(\mathbb{R}^{2}\right)^{\mathcal{T}} \times \mathbb{R}^{\mathfrak{D}}$. 
In order to prove the existence and uniqueness of the solution of (4.1), a discrete Korn inequality is needed and proved in Section 5.1 (see Theorem 5.1).

Proof. Let $N$ denote the cardinal of $\left(\mathbb{R}^{2}\right)^{\mathcal{T}} \times \mathbb{R}^{\mathfrak{D}}$. Scheme (4.1) can be written with $q^{\mathfrak{D}}=0$ and $\alpha=0$ as

$$
\begin{aligned}
\operatorname{div}^{\mathfrak{M}}\left(-2 \eta^{\mathfrak{D}} \mathrm{D}^{\mathfrak{D}} \mathbf{u}^{\mathcal{T}}+p^{\mathfrak{D}} \mathrm{Id}\right) & =\mathbf{f}^{\mathfrak{M}}, \\
\operatorname{div}^{\mathfrak{M}^{*}}\left(-2 \eta^{\mathfrak{D}} \mathrm{D}^{\mathfrak{D}} \mathbf{u}^{\mathcal{T}}+p^{\mathfrak{D}} \mathrm{Id}\right) & =\mathbf{f}^{\mathfrak{M}}, \\
\forall \mathcal{K} \in \partial \mathfrak{M}, \quad \mathbf{u}_{\mathcal{K}} & =\mathbf{g}_{\mathcal{K}}, \\
\forall \mathcal{K}^{*} \in \partial \mathfrak{M}^{*}, \quad \mathbf{u}_{\mathcal{K}^{*}} & =\mathbf{g}_{\mathcal{K}^{*}}, \\
\operatorname{div}^{\mathfrak{D}}\left(\mathbf{u}^{\mathcal{T}}\right)-\lambda h_{\mathfrak{D}}^{2} \Delta^{\mathfrak{D}} p^{\mathfrak{D}} & =q^{\mathfrak{D}} \\
\sum_{\mathcal{D} \in \mathfrak{D}} m_{\mathcal{D}} p^{\mathcal{D}} & =\alpha .
\end{aligned}
$$

This is a linear system: $A v=b$ with a rectangle matrix $A \in \mathcal{M}_{N+1, N}(\mathbb{R}), v \in \mathbb{R}^{N}$ and $b=\left(\mathbf{f}^{\mathfrak{M}}, \mathbf{f}^{\mathfrak{M}^{*}}, \mathbf{g}^{\mathcal{T}}, q^{\mathfrak{D}}, \alpha\right)^{\prime} \in \mathbb{R}^{N+1}$. Let $X$ be the following set

$$
X=\left\{\left(\mathbf{f}^{\mathfrak{M}}, \mathbf{f}^{\mathfrak{M}^{*}}, \mathbf{g}^{\mathcal{T}}, q^{\mathfrak{D}}, \alpha\right)^{\prime} \in \mathbb{R}^{N+1}, \sum_{\mathcal{D} \in \mathfrak{D}} m_{\mathcal{D}} q^{\mathcal{D}}=\sum_{\mathcal{D}_{\sigma, \sigma} \in \mathfrak{D}_{e x t}} m_{\sigma} \gamma_{\boldsymbol{\sigma}}\left(\mathbf{g}^{\mathcal{T}}\right) \cdot \overrightarrow{\mathbf{n}}_{\sigma \mathcal{K}}\right\}
$$

with $\operatorname{dim} X=N$. We have that $\left(\mathbf{f}^{\mathfrak{M}}, \mathbf{f}^{\mathfrak{M}^{*}}, \mathbf{g}^{\mathcal{T}}, 0,0\right)^{\prime}$ belongs to $X$, see Remark 4.1. Similarly, we can prove that $\operatorname{Im} A \subset X$. If we prove that the kernel of the matrix $A$ is zero, we conclude that $\operatorname{dim} \operatorname{Im} A=N$ and so $\operatorname{Im} A=X$. Let us then study the kernel of the matrix $A$. Let $\mathbf{u}^{\mathcal{T}} \in \mathbb{E}_{0}$ and $p^{\mathfrak{D}} \in \mathbb{R}^{\mathfrak{D}}$ such that:

$$
\left\{\begin{aligned}
\operatorname{div}^{\mathfrak{M}}\left(-2 \eta^{\mathfrak{D}} \mathrm{D}^{\mathfrak{D}} \mathbf{u}^{\mathcal{T}}+p^{\mathfrak{D}} \mathrm{Id}\right) & =0 \\
\operatorname{div}^{\mathfrak{M}}{ }^{*}\left(-2 \eta^{\mathfrak{D}} \mathrm{D}^{\mathfrak{D}} \mathbf{u}^{\mathcal{T}}+p^{\mathfrak{D}} \mathrm{Id}\right) & =0 \\
\operatorname{div}^{\mathfrak{D}}\left(\mathbf{u}^{\mathcal{T}}\right)-\lambda h_{\mathfrak{D}}^{2} \Delta^{\mathfrak{D}} p^{\mathfrak{D}} & =0 \\
\sum_{\mathcal{D} \in \mathfrak{D}} m_{\mathcal{D}} p^{\mathcal{D}} & =0
\end{aligned}\right.
$$

By definition of $\llbracket \cdot, \cdot \rrbracket_{\mathcal{T}}$ and the fact that $\mathbf{u}^{\mathcal{T}} \in \mathbb{E}_{0}$, we deduce that

$$
\llbracket \operatorname{div}^{\mathcal{T}}\left(-2 \eta^{\mathfrak{D}} \mathrm{D}^{\mathfrak{D}} \mathbf{u}^{\mathcal{T}}+p^{\mathfrak{D}} \mathrm{Id}\right), \mathbf{u}^{\mathcal{T}} \rrbracket_{\mathcal{T}}=0
$$

Using the discrete Stokes formula Theorem 3.1, noting that $\mathbf{u}^{\mathcal{T}} \in \mathbb{E}_{0}$ implies that $\gamma^{\mathcal{T}}\left(\mathbf{u}^{\mathcal{T}}\right)=$ 0 , that $\mathrm{D}^{\mathfrak{D}} \mathbf{u}^{\mathcal{T}}$ is a symmetric tensor, and substituting $\operatorname{div}^{\mathfrak{D}} \mathbf{u}^{\mathcal{T}}=\operatorname{Trace} \nabla^{\mathfrak{D}} \mathbf{u}^{\mathcal{T}}=(\mathrm{Id}$ : $\nabla^{\mathfrak{D}} \mathbf{u}^{\mathcal{T}}$ ), we obtain

$$
\llbracket \operatorname{div}^{\mathcal{T}}\left(-2 \eta^{\mathfrak{D}} \mathrm{D}^{\mathfrak{D}} \mathbf{u}^{\mathcal{T}}+p^{\mathfrak{D}} \mathrm{Id}\right), \mathbf{u}^{\mathcal{T}} \rrbracket_{\mathcal{T}}=\left(2 \eta^{\mathfrak{D}} \mathrm{D}^{\mathfrak{D}} \mathbf{u}^{\mathcal{T}}: \mathrm{D}^{\mathfrak{D}} \mathbf{u}^{\mathcal{T}}\right)_{\mathfrak{D}}-\left(\operatorname{div}^{\mathfrak{D}} \mathbf{u}^{\mathcal{T}}, p^{\mathfrak{D}}\right)_{\mathfrak{D}}
$$

Furthermore, the mass conservation equation and Remark 3.6 give:

$$
-\left(\operatorname{div}^{\mathfrak{D}} \mathbf{u}^{\mathcal{T}}, p^{\mathfrak{D}}\right)_{\mathfrak{D}}=-\lambda\left(h_{\mathfrak{D}}^{2} \Delta^{\mathfrak{D}} p^{\mathfrak{D}}, p^{\mathfrak{D}}\right)_{\mathfrak{D}}=\lambda\left|p^{\mathfrak{D}}\right|_{h}^{2}
$$

where $|\cdot|_{h}$ is the semi-norm introduced in (3.10). Using the discrete Korn inequality, c.f. Theorem 5.1, and the bounds on $\eta$ given in (2.3), we have:

$$
\llbracket \operatorname{div}^{\mathcal{T}}\left(-2 \eta^{\mathfrak{D}} \mathrm{D}^{\mathfrak{D}} \mathbf{u}^{\mathcal{T}}+p^{\mathfrak{D}} \mathrm{Id}\right), \mathbf{u}^{\mathcal{T}} \rrbracket \mathcal{T} \geq \underline{\mathrm{C}}_{\eta}\left\|\nabla^{\mathfrak{D}} \mathbf{u}^{\mathcal{T}}\right\|_{2}^{2}+\lambda\left|p^{\mathfrak{D}}\right|_{h}^{2}
$$


We finally get

$$
0 \geq \underline{\mathrm{C}}_{\eta}\left\|\nabla^{\mathcal{D}} \mathbf{u}^{\mathcal{T}}\right\|_{2}^{2}+\lambda\left|p^{\mathfrak{D}}\right|_{h}^{2},
$$

which implies that

$$
\left\|\nabla^{\mathcal{D}} \mathbf{u}^{\mathcal{T}}\right\|_{2}^{2}=0 \quad \text { and } \quad\left|p^{\mathfrak{D}}\right|_{h}^{2}=0 .
$$

Therefore, we have that $\nabla^{\mathcal{D}} \mathbf{u}^{\mathcal{T}}=0$ and $p^{\mathfrak{D}}$ is constant, which implies that there exists three constants $\mathbf{c}_{\boldsymbol{0}} \in \mathbb{R}^{2}, \mathbf{c}_{\mathbf{1}} \in \mathbb{R}^{2}$ and $c_{3} \in \mathbb{R}$ such that :

$$
\begin{array}{lrl}
\forall \mathcal{K} \in(\mathfrak{M} \cup \partial \mathfrak{M}), & \mathbf{u}_{\mathcal{K}} & =\mathbf{c}_{\mathbf{0}}, \\
\forall \mathcal{K}^{*} \in\left(\mathfrak{M}^{*} \cup \partial \mathfrak{M}^{*}\right), & \mathbf{u}_{\mathcal{K}^{*}} & =\mathbf{c}_{1}, \\
\forall \mathcal{D} \in \mathfrak{D}, & p^{\mathcal{D}} & =c_{3} .
\end{array}
$$

Since $\mathbf{u}^{\boldsymbol{\tau}}$ belongs to $\mathbb{E}_{0}$ we have $\mathbf{c}_{\boldsymbol{0}}=\mathbf{c}_{\boldsymbol{1}}=0$. Consequently, we have $\mathbf{u}^{\boldsymbol{\tau}}=\mathbf{0}$. Then, we use the fact that $p^{\mathfrak{D}}$ verifies $\sum_{\mathcal{D} \in \mathfrak{D}} m_{\mathcal{D}} p^{\mathcal{D}}=0$ so $p^{\mathfrak{D}}=0$.

5. Results on discrete operators. In this section, we present several results on the discrete operators. In Section 5.1, we begin with the properties of the discrete strain rate tensor. The main result is the proof of a discrete Korn inequality. In Sections 5.2-5.3, we review the results of [2] and adapt them to the vector-valued setting. Then, in Sections 5.4-5.5, we focus on the properties of mean-value projection operator.

5.1. Properties of discrete strain rate tensor. Korn inequality. We first have the bound of the discrete strain rate tensor by the discrete gradient.

Proposition 5.1. For all $\mathbf{u}^{\mathcal{T}} \in\left(\mathbb{R}^{2}\right)^{\mathcal{T}}$, we get

$$
\left\|\mathrm{D}^{\mathfrak{D}} \mathbf{u}^{\mathcal{T}}\right\|_{2} \leq\left\|\nabla^{\mathfrak{D}} \mathbf{u}^{\mathcal{T}}\right\|_{2} .
$$

Proof. Thanks to Remark 2.1 we have

$$
\left\|D^{\mathcal{D}} \mathbf{u}^{\mathcal{T}}\right\|_{2}^{2}=\sum_{\mathcal{D} \in \mathcal{D}} m_{\mathcal{D}}\left\|D^{\mathcal{D}} \mathbf{u}^{\mathcal{T}}\right\|_{\mathcal{F}}^{2} \leq \sum_{\mathcal{D} \in \mathcal{D}} m_{\mathcal{D}}\left\|\nabla^{\mathcal{D}} \mathbf{u}^{\mathcal{T}}\right\|_{\mathcal{F}}^{2}=\left\|\nabla^{\mathcal{D}} \mathbf{u}^{\mathcal{T}}\right\|_{2}^{2} .
$$

We introduce the following notation

$$
\forall \mathbf{u}, \mathbf{v} \in \mathbb{R}^{2}, \quad \mathbf{u} \wedge \mathbf{v}=u_{1} v_{2}-u_{2} v_{1} .
$$

From usual differential calculus, we know that for any smooth function $\mathbf{u}: \Omega \longrightarrow \mathbb{R}^{2}$, we have

$$
\operatorname{div}\left({ }^{t}(\nabla \mathbf{u})\right)=\operatorname{div}(\operatorname{div}(\mathbf{u}) \operatorname{Id})=\nabla(\operatorname{div}(\mathbf{u})) .
$$

The corresponding discrete property is proved in the following proposition.

Proposition 5.2. For all $\mathbf{u}^{\mathcal{T}} \in \mathbb{E}_{0}$, we have

$$
\operatorname{div}^{\mathcal{T}}\left({ }^{t}\left(\nabla^{\mathcal{D}} \mathbf{u}^{\mathcal{T}}\right)\right)=\operatorname{div}^{\mathcal{T}}\left(\operatorname{div}^{\mathfrak{D}}\left(\mathbf{u}^{\mathcal{T}}\right) \operatorname{Id}\right) .
$$

Proof. On any diamond $\mathcal{D} \in \mathfrak{D}$, the matrix $\left({ }^{t}\left(\nabla^{\mathcal{D}} \mathbf{u}^{\mathcal{T}}\right)-\operatorname{div}^{\mathcal{D}} \mathbf{u}^{\mathcal{T}} \mathrm{Id}\right)$ is given by

$$
\left({ }^{t}\left(\nabla^{\mathcal{D}} \mathbf{u}^{\mathcal{T}}\right)-\operatorname{div}^{\mathcal{D}} \mathbf{u}^{\mathcal{T}} \mathrm{Id}\right)=\left(\begin{array}{cc}
-\left(\nabla^{\mathcal{D}} u_{2}^{\mathcal{T}}\right)_{2} & \left(\nabla^{\mathcal{D}} u_{2}^{\mathcal{T}}\right)_{1} \\
\left(\nabla^{\mathcal{D}} u_{1}^{\mathcal{T}}\right)_{2} & -\left(\nabla^{\mathcal{D}} u_{1}^{\mathcal{T}}\right)_{1}
\end{array}\right) .
$$


Let $\mathcal{K} \in \mathfrak{M}$. We obtain

$$
m_{\mathcal{K}} \operatorname{div}^{\mathcal{K}}\left({ }^{t}\left(\nabla^{\mathfrak{D}} \mathbf{u}^{\mathcal{T}}\right)-\operatorname{div}^{\mathfrak{D}}\left(\mathbf{u}^{\mathcal{T}}\right) \mathrm{Id}\right)=\sum_{\mathcal{D} \in \mathfrak{D}_{\mathcal{K}}} m_{\sigma}\left(\begin{array}{c}
\nabla^{\mathcal{D}} u_{2}^{\mathcal{T}} \wedge \overrightarrow{\mathbf{n}}_{\boldsymbol{F} \mathcal{K}} \\
-\nabla^{\mathcal{D}} u_{1}^{\mathcal{T}} \wedge \overrightarrow{\mathbf{n}}_{\sigma \mathcal{K}}
\end{array}\right) .
$$

Using the definition of the gradient for a scalar field defined in Remark 3.3 and the fact that $\overrightarrow{\mathbf{n}}_{\sigma \mathcal{K}} \wedge \overrightarrow{\mathbf{n}}_{\sigma^{*} \mathcal{K}^{*}}=\sin \left(\alpha_{\mathcal{D}}\right)$, we deduce

$$
\nabla^{\mathcal{D}} u_{i}^{\mathcal{T}} \wedge \overrightarrow{\mathbf{n}}_{\sigma \mathcal{K}}=\frac{u_{i, \mathcal{L}^{*}}-u_{i, \mathcal{K}^{*}}}{\sin \left(\alpha_{\mathcal{D}}\right) m_{\sigma}} \overrightarrow{\mathbf{n}}_{\sigma^{*} \mathcal{K}^{*}} \wedge \overrightarrow{\mathbf{n}}_{\sigma \mathcal{K}}=\frac{u_{i, \mathcal{K}^{*}}-u_{i, \mathcal{L}^{*}}}{m_{\sigma}}
$$

It implies that we have

$$
m_{\mathcal{K}} \operatorname{div}^{\mathcal{K}}\left({ }^{t}\left(\nabla^{\mathfrak{D}} \mathbf{u}^{\mathcal{T}}\right)-\operatorname{div}^{\mathfrak{D}}\left(\mathbf{u}^{\mathcal{T}}\right) \mathrm{Id}\right)=\sum_{\mathcal{D} \in \mathfrak{D}_{\mathcal{K}}}\left(\begin{array}{l}
u_{2, \mathcal{K}^{*}}-u_{2, \mathcal{L}^{*}} \\
u_{1, \mathcal{L}^{*}}-u_{1, \mathcal{K}^{*}}
\end{array}\right)
$$

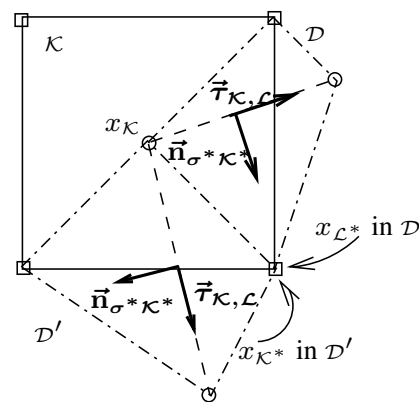

FIG. 5.1. Trigonometrical path.

Recall that in each diamond cell $\mathcal{D} \in \mathfrak{D}_{\mathcal{K}}$, the basis $\left(\overrightarrow{\boldsymbol{\tau}}_{\mathcal{K}, \mathcal{L}}, \overrightarrow{\mathbf{n}}_{\sigma^{*} \mathcal{K}^{*}}\right)$ is supposed to be directly oriented. It implies that for each diamond cell $\mathcal{D} \in \mathfrak{D}_{\mathcal{K}}$ the points $x_{\mathcal{K}^{*}}$ and $x_{\mathcal{L}^{*}}$ are well defined with this choice and for two distinguished diamond cells $\mathcal{D}$ and $\mathcal{D}^{\prime}$, such that $\overline{\mathcal{D}} \cap \overline{\mathcal{D}}^{\prime} \neq \emptyset$, the point $x_{\mathcal{L}^{*}}$ of $\mathcal{D}$ coincides with the point $x_{\mathcal{K}^{*}}$ of $\mathcal{D}^{\prime}$, (see Figure 5.1). Thus the right-hand side of (5.1) is equal to zero. Hence for all $\mathcal{K} \in \mathfrak{M}$, we conclude

$$
\operatorname{div}^{\mathcal{K}}\left({ }^{t}\left(\nabla^{\mathfrak{D}} \mathbf{u}^{\mathcal{T}}\right)\right)=\operatorname{div}^{\mathcal{K}}\left(\operatorname{div}^{\mathfrak{D}}\left(\mathbf{u}^{\mathcal{T}}\right) \operatorname{Id}\right)
$$

The same result holds for all $\mathcal{K}^{*} \in \mathfrak{M}^{*}$. First, we get

$$
m_{\mathcal{K}^{*}} \operatorname{div}^{\mathcal{K}^{*}}\left({ }^{t}\left(\nabla^{\mathfrak{D}} \mathbf{u}^{\mathcal{T}}\right)-\operatorname{div}^{\mathfrak{D}}\left(\mathbf{u}^{\mathcal{T}}\right) \mathrm{Id}\right)=\sum_{\mathcal{D} \in \mathfrak{D}_{\mathcal{K}^{*}}} m_{\sigma^{*}}\left(\begin{array}{c}
\nabla^{\mathcal{D}} u_{2}^{\mathcal{T}} \wedge \overrightarrow{\mathbf{n}}_{\sigma^{*} \mathcal{K}^{*}} \\
-\nabla^{\mathcal{D}} u_{1}^{\mathcal{T}} \wedge \overrightarrow{\mathbf{n}}_{\sigma^{*} \mathcal{K}^{*}}
\end{array}\right),
$$

and

$$
m_{\mathcal{K}^{*}} \operatorname{div}^{\mathcal{K}^{*}}\left({ }^{t}\left(\nabla^{\mathfrak{D}} \mathbf{u}^{\mathcal{T}}\right)-\operatorname{div}^{\mathfrak{D}}\left(\mathbf{u}^{\mathcal{T}}\right) \mathrm{Id}\right)=\sum_{\mathcal{D} \in \mathfrak{D}_{\mathcal{K}^{*}}}\left(\begin{array}{l}
u_{2, \mathcal{L}}-u_{2, \mathcal{K}} \\
u_{1, \mathcal{K}}-u_{1, \mathcal{L}}
\end{array}\right) .
$$

Thanks to the orientation, the right-hand side of (5.2) is equal to zero, it implies that

$$
\operatorname{div}^{\mathcal{K}^{*}}\left({ }^{t}\left(\nabla^{\mathfrak{D}} \mathbf{u}^{\mathcal{T}}\right)\right)=\operatorname{div}^{\mathcal{K}^{*}}\left(\operatorname{div}^{\mathfrak{D}}\left(\mathbf{u}^{\mathcal{T}}\right) \operatorname{Id}\right), \quad \forall \mathcal{K}^{*} \in \mathfrak{M}^{*}
$$


The case where $\mathcal{K}^{*} \in \partial \mathfrak{M}^{*}$ is slightly different, Definition 3.2 of the discrete divergence gives

$$
\begin{aligned}
& m_{\mathcal{K}^{*}} \operatorname{div}^{\mathcal{K}^{*}}\left({ }^{t}\left(\nabla^{\mathcal{D}} \mathbf{u}^{\mathcal{T}}\right)-\operatorname{div}^{\mathfrak{D}}\left(\mathbf{u}^{\mathcal{T}}\right) \mathrm{Id}\right)=\sum_{\mathcal{D} \in \mathfrak{D}_{\mathcal{K}^{*}}} m_{\sigma^{*}}\left({ }^{t}\left(\nabla^{\mathcal{D}} \mathbf{u}^{\mathcal{T}}\right)-\operatorname{div}^{\mathcal{D}} \mathbf{u}^{\mathcal{T}} \mathrm{Id}\right) \overrightarrow{\mathbf{n}}_{\sigma^{*} \mathcal{K}^{*}} \\
& +\sum_{\mathcal{D} \in \mathfrak{D}_{\mathcal{K}^{*}} \cap \mathcal{D}_{\text {ext }}} d_{\mathcal{K}^{*}, \mathcal{L}}\left({ }^{t}\left(\nabla^{\mathcal{D}} \mathbf{u}^{\mathcal{T}}\right)-\operatorname{div}^{\mathcal{D}} \mathbf{u}^{\mathcal{T}} \mathrm{Id}\right) \overrightarrow{\mathbf{n}}_{\sigma \mathcal{K}} .
\end{aligned}
$$

For the first sum on $\mathcal{D} \in \mathfrak{D}_{\mathcal{K}^{*}}$ of the right-hand side of (5.3), we can do the same as for $\mathcal{K}^{*} \in \mathfrak{M}^{*}$ : thanks to (5.2), we have

$$
\sum_{\mathcal{D} \in \mathfrak{D}_{\mathcal{K}^{*}}} m_{\sigma^{*}}\left({ }^{t}\left(\nabla^{\mathcal{D}} \mathbf{u}^{\mathcal{T}}\right)-\operatorname{div}^{\mathcal{D}} \mathbf{u}^{\mathcal{T}} \mathrm{Id}\right) \overrightarrow{\mathbf{n}}_{\sigma^{*} \mathcal{K}^{*}}=\sum_{\mathcal{D} \in \mathfrak{D}_{\mathcal{K}^{*}}}\left(\begin{array}{l}
u_{2, \mathcal{L}}-u_{2, \mathcal{K}} \\
u_{1, \mathcal{K}}-u_{1, \mathcal{L}}
\end{array}\right) .
$$

For the second sum of the right-hand side of (5.3), we can do the same as for $\mathcal{K} \in \mathfrak{M}$ : thanks to (5.1), we have

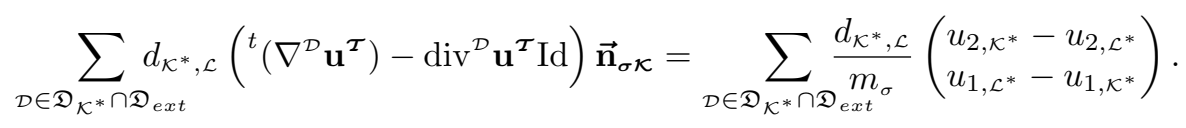

Substituting (5.4) and (5.5) into (5.3), we get

$$
\begin{aligned}
& m_{\mathcal{K}^{*}} \operatorname{div}^{\mathcal{K}^{*}}\left({ }^{t}\left(\nabla^{\mathfrak{D}} \mathbf{u}^{\mathcal{T}}\right)-\operatorname{div}^{\mathfrak{D}}\left(\mathbf{u}^{\mathcal{T}}\right) \mathrm{Id}\right) \\
& =\sum_{\mathcal{D} \in \mathfrak{D}_{\mathcal{K}^{*}}}\left(\begin{array}{l}
u_{2, \mathcal{L}}-u_{2, \mathcal{K}} \\
u_{1, \mathcal{K}}-u_{1, \mathcal{L}}
\end{array}\right)+\sum_{\mathcal{D} \in \mathfrak{D}_{\mathcal{K}^{*}} \cap \mathfrak{D}_{\text {ext }}} \frac{d_{\mathcal{K}^{*}, \mathcal{L}}}{m_{\sigma}}\left(\begin{array}{l}
u_{2, \mathcal{L}^{*}}-u_{2, \mathcal{L}^{*}} \\
u_{1, \mathcal{L}^{*}}-u_{1, \mathcal{K}^{*}}
\end{array}\right) .
\end{aligned}
$$

Thanks to the boundary condition $\mathbf{u}^{\mathcal{T}} \in \mathbb{E}_{0}$, for all $\mathcal{D} \in \mathfrak{D}_{\mathcal{K}^{*}} \cap \mathfrak{D}_{\text {ext }}$, we have $u_{2, \mathcal{K}^{*}}=$ $u_{2, \mathcal{L}^{*}}=u_{1, \mathcal{L}^{*}}=u_{1, \mathcal{K}^{*}}=0$. It implies that

$$
\sum_{\mathcal{D} \in \mathfrak{D}_{\mathcal{K}^{*} \cap \mathfrak{D}_{e x t}}} \frac{d_{\mathcal{K}^{*}, \mathcal{L}}}{m_{\sigma}}\left(\begin{array}{c}
u_{2, \mathcal{K}^{*}}-u_{2, \mathcal{L}^{*}} \\
u_{1, \mathcal{L}^{*}}-u_{1, \mathcal{K}^{*}}
\end{array}\right)=0 .
$$

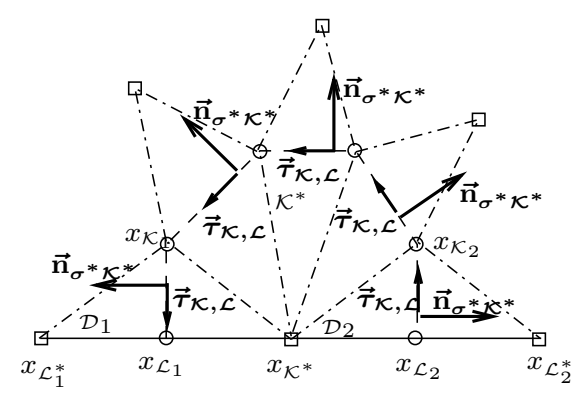

FIG. 5.2. Trigonometrical path.

Using the notation of Figure 5.2, the sum over the diamond cells $\mathcal{D} \in \mathfrak{D}_{\mathcal{K}^{*}}$ is equal to

$$
\sum_{\mathcal{D} \in \mathfrak{D}_{\mathcal{K}^{*}}}\left(\begin{array}{l}
u_{2, \mathcal{L}}-u_{2, \mathcal{K}} \\
u_{1, \mathcal{K}}-u_{1, \mathcal{L}}
\end{array}\right)=\left(\begin{array}{l}
u_{2, \mathcal{L}_{1}}-u_{2, \mathcal{L}_{2}} \\
u_{1, \mathcal{L}_{2}}-u_{1, \mathcal{L}_{1}}
\end{array}\right)
$$


Since $\mathbf{u}^{\mathcal{T}} \in \mathbb{E}_{0}$, we have $u_{2, \mathcal{L}_{1}}=u_{2, \mathcal{L}_{2}}=u_{1, \mathcal{L}_{2}}=u_{1, \mathcal{L}_{1}}=0$. We deduce

$$
\sum_{\mathcal{D} \in \mathfrak{D}_{\mathcal{K}^{*}}}\left(\begin{array}{l}
u_{2, \mathcal{L}}-u_{2, \mathcal{K}} \\
u_{1, \mathcal{L}}-u_{1, \mathcal{L}}
\end{array}\right)+\sum_{\mathcal{D} \in \mathfrak{D}_{\mathcal{K}^{*}} \cap \mathfrak{D}_{e x t}} \frac{d_{\mathcal{K}^{*}, \mathcal{L}}}{m_{\sigma}}\left(\begin{array}{l}
u_{2, \mathcal{K}^{*}}-u_{2, \mathcal{L}^{*}} \\
u_{1, \mathcal{L}^{*}}-u_{1, \mathcal{K}^{*}}
\end{array}\right)=0 .
$$

It concludes the proof.

REMARK 5.1. We need $\mathbf{u}^{\mathcal{T}} \in \mathbb{E}_{0}$ to prove $\operatorname{div}^{\kappa^{*}}\left({ }^{t}\left(\nabla^{\mathfrak{D}} \mathbf{u}^{\mathcal{T}}\right)\right)=\operatorname{div}^{\kappa^{*}}\left(-\operatorname{div}^{\mathfrak{D}}\left(\mathbf{u}^{\mathcal{T}}\right) \operatorname{Id}\right)$, for all $\mathcal{K}^{*} \in \partial \mathfrak{M}^{*}$, otherwise we have to add boundary terms.

From Proposition 5.2, we deduce the following proposition.

Proposition 5.3. For all $\mathbf{u}^{\tau} \in \mathbb{E}_{0}$, we have

$$
\left({ }^{t}\left(\nabla^{\mathcal{D}} \mathbf{u}^{\mathcal{T}}\right): \nabla^{\mathfrak{D}} \mathbf{u}^{\mathcal{T}}\right)_{\mathfrak{D}}=\left\|\operatorname{div}^{\mathfrak{D}}\left(\mathbf{u}^{\mathcal{T}}\right)\right\|_{2}^{2} \geq 0 .
$$

Proof. Using the Stokes formula Theorem 3.1, the fact that $\mathbf{u}^{\mathcal{T}} \in \mathbb{E}_{0}$ and then Proposition 5.2, we have

$$
\left({ }^{t}\left(\nabla^{\mathfrak{D}} \mathbf{u}^{\mathcal{T}}\right): \nabla^{\mathfrak{D}} \mathbf{u}^{\mathcal{T}}\right)_{\mathfrak{D}}=-\llbracket \operatorname{div}^{\mathcal{T}}\left({ }^{t}\left(\nabla^{\mathfrak{D}} \mathbf{u}^{\mathcal{T}}\right)\right), \mathbf{u}^{\mathcal{T}} \rrbracket_{\mathcal{T}}=-\llbracket \operatorname{div}^{\mathcal{T}}\left(\operatorname{div}^{\mathfrak{D}}\left(\mathbf{u}^{\mathcal{T}}\right) \operatorname{Id}\right), \mathbf{u}^{\mathcal{T}} \rrbracket_{\mathcal{T}} .
$$

Using once more the Stokes formula Theorem 3.1 for $\mathbf{u}^{\mathcal{T}} \in \mathbb{E}_{0}$ and substituting $\operatorname{div}^{\mathfrak{D}} \mathbf{u}^{\mathcal{T}}=$ $\operatorname{Trace} \nabla^{\mathfrak{D}} \mathbf{u}^{\mathcal{T}}=\left(\operatorname{Id}: \nabla^{\mathfrak{D}} \mathbf{u}^{\mathcal{T}}\right)$, we obtain

$$
\left({ }^{t}\left(\nabla^{\mathcal{D}} \mathbf{u}^{\mathcal{T}}\right): \nabla^{\mathfrak{D}} \mathbf{u}^{\mathcal{T}}\right)_{\mathfrak{D}}=\left(\operatorname{div}^{\mathfrak{D}}\left(\mathbf{u}^{\mathcal{T}}\right) \operatorname{Id}: \nabla^{\mathfrak{D}} \mathbf{u}^{\mathcal{T}}\right)_{\mathfrak{D}}=\left\|\operatorname{div}^{\mathfrak{D}}\left(\mathbf{u}^{\mathcal{T}}\right)\right\|_{2}^{2} \geq 0 .
$$

We are now able to prove a discrete Korn inequality:

THEOREM 5.1 (Discrete Korn inequality). For all $\mathbf{u}^{\mathcal{T}} \in \mathbb{E}_{0}$, we have

$$
\left\|\nabla^{\mathcal{D}} \mathbf{u}^{\mathcal{T}}\right\|_{2} \leq \sqrt{2}\left\|D^{\mathfrak{D}} \mathbf{u}^{\mathcal{T}}\right\|_{2} .
$$

Proof. This is just a consequence of the following equality and of Proposition 5.3.

$$
\left\|D^{\mathfrak{D}} \mathbf{u}^{\mathcal{T}}\right\|_{2}^{2}=\frac{1}{2}\left\|\nabla^{\mathfrak{D}} \mathbf{u}^{\mathcal{T}}\right\|_{2}^{2}+\frac{1}{2}\left({ }^{t}\left(\nabla^{\mathfrak{D}} \mathbf{u}^{\mathcal{T}}\right): \nabla^{\mathfrak{D}} \mathbf{u}^{\mathcal{T}}\right)_{\mathfrak{D}} .
$$

5.2. Technical lemmas. Poincaré inequality. We will need two technical results and the discrete Poincare inequality whose proofs can be found in the literature. The first one is [2, Lemma 3.4] (see also [17, Lemma 6.3]).

LEMMA 5.1. There exists a constant $C>0$ such that for any bounded polygonal set $\mathcal{P} \subset \mathbb{R}^{2}$ with positive measure, any segment $\sigma \subset \mathbb{R}^{2}$ and any $v \in H^{1}\left(\mathbb{R}^{2}\right)$, we have

$$
\left|v_{\mathcal{P}}-v_{\sigma}\right|^{2} \leq \frac{1}{m_{\sigma} m_{\mathcal{P}}} \int_{\sigma} \int_{\mathcal{P}}|v(x)-v(y)|^{2} \mathrm{~d} x \mathrm{~d} y \leq C \frac{\operatorname{diam}\left(\widehat{\mathcal{P}_{\sigma}}\right)^{3}}{m_{\sigma} m_{\mathcal{P}}} \int_{\widehat{\mathcal{P}_{\sigma}}}|\nabla v(z)|^{2} \mathrm{~d} z,
$$

where $v_{\mathcal{P}}$ denotes the mean value of $v$ on $\mathcal{P}, v_{\sigma}$ the mean value of $v$ on the segment $\sigma$, and $\widehat{\mathcal{P}_{\sigma}}$ is the convex hull of $\mathcal{P} \cup \sigma$.

The second one is the vector-valued version of the first Lemma 8.1 in the Appendix [16].

LEMMA 5.2. Let $K$ be a non empty open polygonal convex set in $\mathbb{R}^{2}$ such that, for some $\alpha>0$, there exists a ball of radius $\alpha \operatorname{diam}(K)$ contained in $K$. Let $E$ be an affine 
hyperplane of $\mathbb{R}^{2}$ and $\sigma$ be a non-empty open subset of $E$ contained in $\partial K \cap E$. Then there exists a constant $C>0$, depending only on $\alpha$, for any $\mathbf{v} \in\left(H^{1}(K)\right)^{2}$ :

$$
\left|\frac{1}{m_{\sigma}} \int_{\sigma} \mathbf{v}(s) \mathrm{d} s\right|^{2} \leq \frac{C \operatorname{diam}(K)}{m_{\sigma}} \int_{K}\|\nabla \mathbf{v}(s)\|_{\mathcal{F}}^{2} \mathrm{~d} s+\frac{C}{\operatorname{diam}(K) m_{\sigma}} \int_{K}|\mathbf{v}(s)|^{2} \mathrm{~d} s .
$$

Let us finally state the discrete version of the Poincare inequality which is the vectorvalued version of [2, Lemma 3.3].

THEOREM 5.2 (Discrete Poincaré inequality). Let $\mathcal{T}$ be a mesh of $\Omega$. There exists $a$ constant $C>0$, depending only on the diameter of $\Omega$ and $\operatorname{reg}(\mathcal{T})$, such that for any $\mathbf{u}^{\mathcal{T}} \in \mathbb{E}_{0}$, we have

$$
\left\|\mathbf{u}^{\mathcal{T}}\right\|_{2} \leq C\left\|\nabla^{\mathfrak{D}} \mathbf{u}^{\mathcal{T}}\right\|_{2}
$$

5.3. Properties of the mean-value and center-value projection operators. In this subsection, we give some lemmas on projection operators. We only prove results which can not be deduced immediately from their scalar counterpart (given in [2]) by working component per component.

The following properties of the center-value projection operator, defined by (3.6), are used in the estimate of the consistency error of our finite volume scheme.

LEMMA 5.3. Let $\mathcal{T}$ be a mesh of $\Omega$. There exists a constant $C>0$, depending only on $\operatorname{reg}(\mathcal{T})$, such that for any function $\mathbf{v}$ in $\left(H^{2}(\Omega)\right)^{2}$, we have

$$
\left\|\nabla \mathbf{v}-\nabla^{\mathfrak{D}} \mathbb{P}_{\boldsymbol{c}}^{\mathcal{T}} \mathbf{v}\right\|_{2} \leq C \operatorname{size}(\mathcal{T})\|\nabla \mathbf{v}\|_{H^{1}}
$$

COROLlary 5.1. Let $\mathcal{T}$ be a mesh of $\Omega$. There exists a constant $C>0$, depending only on $\operatorname{reg}(\mathcal{T})$, such that for any function $\mathrm{v}$ in $\left(H^{2}(\Omega)\right)^{2}$, we have

$$
\left\|\nabla^{\mathfrak{D}} \mathbb{P}_{\boldsymbol{c}}^{\mathcal{T}} \mathbf{v}\right\|_{2} \leq C\|\nabla \mathbf{v}\|_{H^{1}}
$$

COROLlary 5.2. Let $\mathcal{T}$ be a mesh of $\Omega$. There exists a constant $C>0$, depending only on $\operatorname{reg}(\mathcal{T})$, such that for any function $\mathbf{v}$ in $\left(H^{2}(\Omega)\right)^{2}$ which satisfies $\operatorname{div} \mathbf{v}=0$, we have

$$
\left\|\operatorname{div}^{\mathfrak{D}} \mathbb{P}_{\boldsymbol{c}}^{\mathcal{T}} \mathbf{v}\right\|_{2} \leq C \operatorname{size}(\mathcal{T})\|\nabla \mathbf{v}\|_{H^{1}}
$$

Proof. Let $\mathbf{v} \in\left(H^{2}(\Omega)\right)^{2}$ which satisfies $\operatorname{div} \mathbf{v}=0$. Let $\mathcal{D} \in \mathfrak{D}$; using the fact that $\operatorname{div}^{\mathcal{D}}\left(\mathbb{P}_{\boldsymbol{c}}^{\mathcal{T}} \mathbf{v}\right)=\operatorname{Trace}\left(\nabla^{\mathcal{D}} \mathbb{P}_{\boldsymbol{c}}^{\mathcal{T}} \mathbf{v}\right)$ and $\operatorname{div} \mathbf{v}=\operatorname{Trace}(\nabla \mathbf{v})=0$, we have

$$
\operatorname{div}^{\mathcal{D}}\left(\mathbb{P}_{c}^{\mathcal{T}} \mathbf{v}\right)=\operatorname{Trace}\left(\nabla^{\mathcal{D}} \mathbb{P}_{\boldsymbol{c}}^{\mathcal{T}} \mathbf{v}-\nabla \mathbf{v}(x)\right), \quad \forall x \in \mathcal{D} .
$$

Lemma 5.3 gives

$$
\left\|\operatorname{div}^{\mathfrak{D}}\left(\mathbb{P}_{\boldsymbol{c}}^{\mathcal{T}} \mathbf{v}\right)\right\|_{2} \leq\left\|\nabla^{\mathfrak{D}} \mathbb{P}_{\boldsymbol{c}}^{\mathcal{T}} \mathbf{v}-\nabla \mathbf{v}\right\|_{2} \leq C \operatorname{size}(\mathcal{T})\|\nabla \mathbf{v}\|_{H^{1}}
$$

We will need to evaluate the contribution in the error of the two different projections $\mathbb{P}_{\boldsymbol{c}}^{\mathcal{T}} \mathbf{v}, \mathfrak{P}_{\boldsymbol{m}, \boldsymbol{g}} \mathbb{P}_{\boldsymbol{c}}^{\mathcal{T}} \mathbf{v}$, defined by (3.6) and (3.8), where $\mathbf{g}=\gamma(\mathbf{v})$. Note that these two projections only differ on the boundary cells. For Lemma 5.4 it is crucial to have that $\mathbb{P}_{\boldsymbol{m}}^{\partial \Omega}$, defined 
by (3.3), used the mean-value on $\sigma_{\mathcal{K}}$ (resp. $\left.\sigma_{\mathcal{K}^{*}}\right)$ with the point $x_{\mathcal{K}}$ (resp. $\left.x_{\mathcal{K}^{*}}\right)$ located at the middle of the edge $\sigma_{\mathcal{K}}$ (resp. $\sigma_{\mathcal{K}^{*}}$ ).

LEMMA 5.4. Let $\mathcal{T}$ be a mesh of $\Omega$. There exists a constant $C>0$, depending only on $\operatorname{reg}(\mathcal{T})$, such that for any function $\mathbf{v}$ in $\left(H^{2}(\Omega)\right)^{2}$, whose trace is denoted by $\mathbf{g}=\gamma(\mathbf{v})$, we have

$$
\left\|\nabla^{\mathfrak{D}} \mathbb{P}_{\boldsymbol{c}}^{\mathcal{T}} \mathbf{v}-\nabla^{\mathfrak{D}} \mathfrak{P}_{\boldsymbol{m}, \boldsymbol{g}} \mathbb{P}_{\boldsymbol{c}}^{\mathcal{T}} \mathbf{v}\right\|_{2} \leq C \operatorname{size}(\mathcal{T})\|\mathbf{v}\|_{H^{2}}
$$

Next lemma gives the main properties of the mean-value projection, defined by (3.5), of a $H^{1}$ functions.

LEMMA 5.5. Let $\mathcal{T}$ be a mesh of $\Omega$. There exists $C>0$ depending only on $\operatorname{reg}(\mathcal{T})$, such that:

$$
\begin{aligned}
\left\|\nabla^{\mathfrak{D}} \mathbb{P}_{\boldsymbol{m}}^{\mathcal{T}} \mathbf{v}\right\|_{2} & \leq C\|\nabla \mathbf{v}\|_{2}, \quad \forall \mathbf{v} \in\left(H^{1}(\Omega)\right)^{2}, \\
\left\|\mathbf{v}-\mathbb{P}_{\boldsymbol{m}}^{\mathcal{T}} \mathbf{v}\right\|_{2} & \leq C \operatorname{size}(\mathcal{T})\|\nabla \mathbf{v}\|_{2}, \quad \forall \mathbf{v} \in\left(H^{1}(\Omega)\right)^{2}
\end{aligned}
$$

We give below the main properties of the center-value projection, defined by (3.6).

LEMMA 5.6. Let $\mathcal{T}$ be a mesh of $\Omega$. There exists $C>0$ depending only on $\operatorname{reg}(\mathcal{T})$, such that:

$$
\begin{array}{rlrl}
\left\|\mathbf{v}-\mathbb{P}_{\boldsymbol{c}}^{\mathcal{T}} \mathbf{v}\right\|_{2} \leq C \operatorname{size}(\mathcal{T})\|\nabla \mathbf{v}\|_{H^{1}}, & & \forall \mathbf{v} \in\left(H^{2}(\Omega)\right)^{2}, \\
\left\|\mathbf{v}-\mathfrak{P}_{\boldsymbol{m}, \boldsymbol{g}^{\mathcal{T}}} \mathbb{P}^{\mathcal{T}}\right\|_{2} \leq C \operatorname{size}(\mathcal{T})\|\nabla \mathbf{v}\|_{H^{1}}, & \forall \mathbf{v} \in\left(H^{2}(\Omega)\right)^{2},
\end{array}
$$

where $\mathbf{g}=\gamma(\mathbf{v})$.

Proof. We only prove the second inequality. The definition of the projection $\mathfrak{P}_{\boldsymbol{m}, \boldsymbol{g}}$ implies that $\mathfrak{P}_{\boldsymbol{m}, \boldsymbol{g}} \mathbb{P}_{\boldsymbol{c}}^{\mathcal{T}} \mathbf{v}$ and $\mathbb{P}_{\boldsymbol{c}}^{\mathcal{T}} \mathbf{v}$ only differ on the boundary $\partial \mathfrak{M}$ and $\partial \mathfrak{M}^{*}$ whereas $\mathfrak{P}_{\boldsymbol{m}, \boldsymbol{g}} \mathbb{P}_{\boldsymbol{c}}^{\mathcal{T}} \mathbf{v}$ and $\mathbb{P}_{\boldsymbol{m}}^{\mathcal{T}} \mathbf{v}$ coincide on the boundary $\partial \mathfrak{M}$ and $\partial \mathfrak{M}^{*}$. So we get

$$
\begin{aligned}
\left\|\mathbf{v}-\mathfrak{P}_{\boldsymbol{m}, \boldsymbol{g}^{\prime}} \mathbb{P}_{\boldsymbol{c}}^{\mathcal{T}} \mathbf{v}\right\|_{2}^{2}= & \frac{1}{2} \sum_{\mathcal{K} \in \mathfrak{M}} \int_{\mathcal{K}}\left|\mathbf{v}(x)-\mathbf{v}\left(x_{\mathcal{K}}\right)\right|^{2} \mathrm{~d} x+\frac{1}{2} \sum_{\mathcal{K}^{*} \in \mathfrak{M}^{*}} \int_{\mathcal{K}^{*}}\left|\mathbf{v}(x)-\mathbf{v}\left(x_{\mathcal{K}^{*}}\right)\right|^{2} \mathrm{~d} x \\
& +\frac{1}{2} \sum_{\mathcal{K}^{*} \in \partial \mathfrak{M}^{*}} \int_{\mathcal{K}^{*}}\left|\mathbf{v}(x)-\frac{1}{m_{\sigma_{\mathcal{K}^{*}}}} \int_{\sigma_{\mathcal{K}^{*}}} \mathbf{v}(z) \mathrm{d} z\right|^{2} \mathrm{~d} x
\end{aligned}
$$

We deduce that

$$
\left\|\mathbf{v}-\mathfrak{P}_{\boldsymbol{m}, \boldsymbol{g}} \mathbb{P}_{\boldsymbol{c}}^{\mathcal{T}} \mathbf{v}\right\|_{2}^{2} \leq\left\|\mathbf{v}-\mathbb{P}_{\boldsymbol{c}}^{\mathcal{T}} \mathbf{v}\right\|_{2}^{2}+\left\|\mathbf{v}-\mathbb{P}_{\boldsymbol{m}}^{\mathcal{T}} \mathbf{v}\right\|_{2}^{2}
$$

Lemma 5.5 and the first inequality conclude the proof.

5.4. Properties of mean-value projection operator on the diamond mesh. The error estimates analysis of our scheme involves the estimate of the projection $\mathbb{P}_{m}^{\mathcal{D}}$ on the diamond mesh, defined by (3.7), of functions lying in $H^{1}(\Omega)$. We give below a property of such a projection onto the set of discrete functions in our framework.

LEMMA 5.7. Let $\mathcal{T}$ be a mesh of $\Omega$. There exists $C>0$ depending only on $\operatorname{reg}(\mathcal{T})$, such that for any function $p$ in $H^{1}(\Omega)$, we have

$$
\sum_{\mathfrak{s}=\mathcal{D} \mid \mathcal{D}^{\prime} \in \mathfrak{S}}\left(\mathbb{P}_{m}^{\mathcal{D}^{\prime}} p-\mathbb{P}_{m}^{\mathcal{D}} p\right)^{2} \leq C\|\nabla p\|_{2}^{2} .
$$


Proof. Let $p \in H^{1}(\Omega)$. We note for simplicity $p^{\mathcal{D}}=\mathbb{P}_{m}^{\mathcal{D}} p$ for any $\mathcal{D} \in \mathfrak{D}$ and $p^{\mathfrak{s}}=$ $\frac{1}{m_{\mathfrak{s}}} \int_{\mathfrak{s}} p(y) \mathrm{d} y$, for any $\mathfrak{s} \in \mathfrak{S}$. We add and subtract $p^{\mathfrak{s}}$ and use the Cauchy-Schwarz inequality:

$$
\sum_{\mathfrak{s}=\mathcal{D} \mid \mathcal{D}^{\prime} \in \mathfrak{S}}\left(p^{\mathcal{D}^{\prime}}-p^{\mathcal{D}}\right)^{2} \leq 2 \sum_{\mathfrak{s}=\mathcal{D} \mid \mathcal{D}^{\prime} \in \mathfrak{S}}\left|p^{\mathcal{D}^{\prime}}-p^{\mathfrak{s}}\right|^{2}+2 \sum_{\mathfrak{s}=\mathcal{D} \mid \mathcal{D}^{\prime} \in \mathfrak{S}}\left|p^{\mathcal{D}}-p^{\mathfrak{s}}\right|^{2} .
$$

Lemma 5.1 applied on a diamond edge $\mathfrak{s}$ and the diamond cell $\mathcal{D}$, leads to

$$
\left|p^{\mathcal{D}}-p^{\mathfrak{s}}\right|^{2} \leq C \frac{h_{\mathcal{D}}^{3}}{m_{\mathfrak{s}} m_{\mathcal{D}}} \int_{\mathcal{D}}|\nabla p(z)|^{2} \mathrm{~d} z
$$

As $\frac{h_{\mathcal{D}}^{3}}{m_{\mathfrak{s}} m_{\mathcal{D}}} \leq C(\operatorname{reg}(\mathcal{T})),($ see the relation $(3.2))$, we obtain

$$
\left|p^{\mathcal{D}}-p^{\mathfrak{s}}\right|^{2} \leq C \int_{\mathcal{D}}|\nabla p(z)|^{2} \mathrm{~d} z .
$$

Substituting (5.7) into (5.6), we get

$$
\sum_{\mathfrak{s}=\mathcal{D} \mid \mathcal{D}^{\prime} \in \mathfrak{S}}\left(p^{\mathcal{D}^{\prime}}-p^{\mathcal{D}}\right)^{2} \leq C \sum_{\mathfrak{s}=\mathcal{D} \mid \mathcal{D}^{\prime} \in \mathfrak{S}}\left(\int_{\mathcal{D}}|\nabla p(z)|^{2} \mathrm{~d} z+\int_{\mathcal{D}^{\prime}}|\nabla p(z)|^{2} \mathrm{~d} z\right) .
$$

A diamond cells has at most four neighbouring diamond cells, we deduce

$$
\sum_{\mathfrak{s}=\mathcal{D} \mid \mathcal{D}^{\prime} \in \mathfrak{S}}\left(p^{\mathcal{D}^{\prime}}-p^{\mathcal{D}}\right)^{2} \leq 4 C \sum_{\mathcal{D} \in \mathfrak{D}} \int_{\mathcal{D}}|\nabla p(z)|^{2} \mathrm{~d} z=4 C \int_{\Omega}|\nabla p(z)|^{2} \mathrm{~d} z .
$$

Proposition 5.4. For any mesh $\mathcal{T}$ on $\Omega$, there exists a constant $C>0$, depending only on $\operatorname{reg}(\mathcal{T})$, such that for any function $p$ in $H^{1}(\Omega)$, we have

$$
\left\|\mathbb{P}_{m}^{\mathfrak{D}} p-p\right\|_{2} \leq C \operatorname{size}(\mathcal{T})\|\nabla p\|_{2} .
$$

Proof. Let $\mathcal{D} \in \mathfrak{D}$. We apply the Jensen inequality

$$
\int_{\mathcal{D}}\left|\mathbb{P}_{m}^{\mathcal{D}} p-p(x)\right|^{2} \mathrm{~d} x \leq \frac{1}{m_{\mathcal{D}}} \int_{\mathcal{D}} \int_{\mathcal{D}}|p(z)-p(x)|^{2} \mathrm{~d} z \mathrm{~d} x .
$$

To get the upper bound, we add and subtract $\frac{1}{m_{\mathfrak{s}}} \int_{\mathfrak{s}} p(y) \mathrm{d} y$ for $\mathfrak{s} \in \mathcal{E}_{\mathcal{D}}$ and use the CauchySchwarz inequality:

$$
\int_{\mathcal{D}}\left|\mathbb{P}_{m}^{\mathcal{D}} p-p(x)\right|^{2} \mathrm{~d} x \leq 4 \int_{\mathcal{D}}\left|\frac{1}{m_{\mathfrak{S}}} \int_{\mathfrak{s}}(p(z)-p(y)) \mathrm{d} y\right|^{2} \mathrm{~d} z .
$$

Applying the Jensen inequality, we have

$$
\int_{\mathcal{D}}\left|\mathbb{P}_{m}^{\mathcal{D}} p-p(x)\right|^{2} \mathrm{~d} x \leq \frac{4}{m_{\mathfrak{s}}} \int_{\mathcal{D}} \int_{\mathfrak{s}}|p(z)-p(y)|^{2} \mathrm{~d} y \mathrm{~d} z .
$$

We apply Lemma 5.1 on a diamond edge $\mathfrak{s}$ and a diamond cell $\mathcal{D}$

$$
\frac{1}{m_{\mathfrak{S}}} \int_{\mathcal{D}} \int_{\mathfrak{s}}|p(y)-p(x)|^{2} \mathrm{~d} y \mathrm{~d} x \leq C \frac{h_{\mathcal{D}}^{3}}{m_{\mathfrak{S}}} \int_{\mathcal{D}}|\nabla p(z)|^{2} \mathrm{~d} z .
$$


The relation (3.2) gives that $\frac{h_{\mathcal{D}}}{m_{\mathfrak{s}}} \leq C$. Finally, we obtain

$$
\int_{\mathcal{D}}\left|\mathbb{P}_{m}^{\mathcal{D}} p-p(x)\right|^{2} \mathrm{~d} x \leq \operatorname{size}(\mathcal{T})^{2} C \int_{\mathcal{D}}|\nabla p(z)|^{2} \mathrm{~d} z
$$

which concludes the proof.

5.5. Properties of the discrete divergence on diamond cells. In order to prove the stability of our finite volume scheme, we will need the following estimate.

PROPOSITION 5.5. Let $\mathcal{T}$ be a mesh of $\Omega$. There exists $C>0$ depending only on $\operatorname{reg}(\mathcal{T})$, such that for any function $\mathbf{v}$ in $\left(H^{1}(\Omega)\right)^{2}$ and any $p^{\mathfrak{D}} \in \mathbb{R}^{\mathfrak{D}}$, we have

$$
\sum_{\mathcal{D} \in \mathfrak{D}} \int_{\mathcal{D}} p^{\mathcal{D}}\left(\operatorname{div}^{\mathcal{D}}\left(\mathbf{v}^{\mathcal{T}}\right)-\operatorname{div}(\mathbf{v})\right) \mathrm{d} z \leq C\left|p^{\mathfrak{D}}\right|_{h}\|\mathbf{v}\|_{H^{1}}
$$

where $\mathbf{v}^{\mathcal{T}}=\mathbb{P}_{m}^{\mathcal{T}} \mathbf{v}$ is the mean-value projection of $\mathbf{v}$, defined by (3.5), on the mesh $\mathcal{T}$.

Proof. Using the equality (3.9) and the continuous Stokes formula, we have, for any $\mathcal{D} \in \mathfrak{D}$ :

$\int_{\mathcal{D}}\left(\operatorname{div}^{\mathcal{D}}\left(\mathbf{v}^{\mathcal{T}}\right)-\operatorname{div}(\mathbf{v}(z))\right) \mathrm{d} z=\sum_{\mathfrak{s}=\left[x_{\mathcal{K}}, x_{\mathcal{K}^{*}}\right] \in \mathcal{E}_{\mathcal{D}}} m_{\mathfrak{s}} \frac{1}{m_{\mathfrak{s}}} \int_{\mathfrak{s}}\left(\frac{\mathbf{v}_{\mathcal{K}}+\mathbf{v}_{\mathcal{K}^{*}}}{2}-\mathbf{v}(z)\right) \cdot \overrightarrow{\mathbf{n}}_{\mathfrak{s} \mathcal{D}} \mathrm{d} z$.

We note $\mathrm{R}_{\text {div }}^{\mathfrak{s}}(\mathbf{v})=\frac{1}{m_{\mathfrak{s}}} \int_{\mathfrak{s}}\left(\frac{\mathbf{v}_{\mathcal{K}}+\mathbf{v}_{\mathcal{K}^{*}}}{2}-\mathbf{v}(z)\right) \mathrm{d} z$. First we multiply by $p^{\mathcal{D}}$ and we sum over the diamond cells $\mathcal{D} \in \mathfrak{D}$.

$$
\sum_{\mathcal{D} \in \mathfrak{D}} \int_{\mathcal{D}} p^{\mathcal{D}}\left(\operatorname{div}^{\mathcal{D}}\left(\mathbf{v}^{\mathcal{T}}\right)-\operatorname{div}(\mathbf{v}(z))\right) \mathrm{d} z=\sum_{\mathcal{D} \in \mathfrak{D}} p^{\mathcal{D}} \sum_{\mathfrak{s} \in \mathcal{E}_{\mathcal{D}}} m_{\mathfrak{s}} \mathrm{R}_{\operatorname{div}}^{\mathfrak{s}}(\mathbf{v}) \cdot \overrightarrow{\mathbf{n}}_{\mathfrak{s} \mathcal{D}}
$$

Reordering the summation over $\mathfrak{s} \in \mathfrak{S}$, we get

$$
\sum_{\mathcal{D} \in \mathfrak{D}} \int_{\mathcal{D}} p^{\mathcal{D}}\left(\operatorname{div}^{\mathcal{D}}\left(\mathbf{v}^{\mathcal{T}}\right)-\operatorname{div}(\mathbf{v}(z))\right) \mathrm{d} z=\sum_{\mathfrak{s}=\mathcal{D} \mid \mathcal{D}^{\prime} \in \mathfrak{S}} m_{\mathfrak{s}} \mathrm{R}_{\operatorname{div}}^{\mathfrak{s}}(\mathbf{v}) \cdot \overrightarrow{\mathbf{n}}_{\mathfrak{s} \mathcal{D}}\left(p^{\mathcal{D}}-p^{\mathcal{D}^{\prime}}\right) .
$$

The Cauchy-Schwarz inequality and (3.10) imply that

$$
\sum_{\mathcal{D} \in \mathfrak{D}} \int_{\mathcal{D}} p^{\mathcal{D}}\left(\operatorname{div}^{\mathcal{D}}\left(\mathbf{v}^{\mathcal{T}}\right)-\operatorname{div}(\mathbf{v}(z))\right) \mathrm{d} z \leq\left|p^{\mathfrak{D}}\right|_{h}\left(\sum_{\mathfrak{s}=\mathcal{D} \mid \mathcal{D}^{\prime} \in \mathfrak{S}} \frac{m_{\mathfrak{s}}^{2}}{h_{\mathcal{D}}^{2}+h_{\mathcal{D}^{\prime}}^{2}}\left|\mathrm{R}_{\operatorname{div}}^{\mathfrak{s}}(\mathbf{v})\right|^{2}\right)^{\frac{1}{2}}
$$

In order to conclude, it remains to prove that

$$
\left(\sum_{\mathfrak{s}=\mathcal{D} \mid \mathcal{D}^{\prime} \in \mathfrak{S}} \frac{m_{\mathfrak{s}}^{2}}{h_{\mathcal{D}}^{2}+h_{\mathcal{D}^{\prime}}^{2}}\left|\mathrm{R}_{\text {div }}^{\mathfrak{s}}(\mathbf{v})\right|^{2}\right)^{\frac{1}{2}} \leq C\|\mathbf{v}\|_{H^{1}},
$$

which is done in the following Lemma 5.8.

LEMMA 5.8. Let $\mathcal{T}$ be a mesh of $\Omega$. There exists $C>0$ depending only on $\operatorname{reg}(\mathcal{T})$, such that for any function $\mathbf{v}$ in $\left(H^{1}(\Omega)\right)^{2}$, we have

$$
\sum_{\mathfrak{s}=\mathcal{D} \mid \mathcal{D}^{\prime} \in \mathfrak{S}} \frac{m_{\mathfrak{s}}^{2}}{h_{\mathcal{D}}^{2}+h_{\mathcal{D}^{\prime}}^{2}}\left|\mathrm{R}_{\mathrm{div}}^{\mathfrak{s}}(\mathbf{v})\right|^{2} \leq C\|\mathbf{v}\|_{H^{1}}^{2},
$$


where $\mathbf{v}^{\mathcal{T}}=\mathbb{P}_{\boldsymbol{m}}^{\mathcal{T}} \mathbf{v}$ and $\mathrm{R}_{\text {div }}^{\mathfrak{s}}(\mathbf{v})=\frac{1}{m_{\mathfrak{s}}} \int_{\mathfrak{s}}\left(\frac{\mathbf{v}_{\mathcal{K}}+\mathbf{v}_{\mathcal{K}^{*}}}{2}-\mathbf{v}(z)\right) \mathrm{d} z$.

Proof. Let $\mathbf{v} \in\left(H^{1}(\Omega)\right)^{2}$. We note $\mathbf{v}=\left(\begin{array}{c}v^{1} \\ v^{2}\end{array}\right)$ and the value $\mathbf{v}_{\mathfrak{s}}:=\frac{1}{m_{\mathfrak{s}}} \int_{\mathfrak{s}} \mathbf{v}(y) \mathrm{d} y$, for any $\mathfrak{s} \in \mathfrak{S}$. We prove the result for any component of $\mathbf{v}$, and then for the function $\mathbf{v}$. For $i=1$, 2. For instance, since $\mathfrak{s} \in \mathfrak{S}$, we can write $\mathfrak{s}=\left[x_{\mathcal{K}}, x_{\mathcal{K}^{*}}\right] \subset \mathcal{K}$, we apply Lemma 5.1 on the edge $\mathfrak{s}$ and on the primal cell $\mathcal{K}$ :

$$
\left|v_{\mathcal{K}}^{i}-v_{\mathfrak{s}}^{i}\right|^{2}=\left|\frac{1}{m_{\mathfrak{s}} m_{\mathcal{K}}} \int_{\mathcal{K}} \int_{\mathfrak{s}}\left(v^{i}(z)-v^{i}(x)\right) \mathrm{d} z \mathrm{~d} x\right|^{2} \leq C \frac{(\operatorname{diam}(\widehat{\mathcal{K}}))^{3}}{m_{\mathfrak{s}} m_{\mathcal{K}}} \int_{\widehat{\mathcal{K}}}\left|\nabla v^{i}(z)\right|^{2} \mathrm{~d} z .
$$

Thanks to the relation (3.2), we have $\frac{m_{\mathfrak{s}}^{2}(\operatorname{diam}(\widehat{\mathcal{K}}))^{3}}{m_{\mathfrak{S}} m_{\mathcal{K}}\left(h_{\mathcal{D}}^{2}+h_{\mathcal{D}^{\prime}}^{2}\right)} \leq C$. It implies that

$$
\frac{m_{\mathfrak{s}}^{2}}{h_{\mathcal{D}}^{2}+h_{\mathcal{D}^{\prime}}^{2}}\left|v_{\mathcal{K}}^{i}-v_{\mathfrak{s}}^{i}\right|^{2} \leq C \int_{\widehat{\mathcal{K}}}\left|\nabla v^{i}(z)\right|^{2} \mathrm{~d} z
$$

We deduce that

$$
\begin{aligned}
\frac{m_{\mathfrak{s}}^{2}}{h_{\mathcal{D}}^{2}+h_{\mathcal{D}^{\prime}}^{2}}\left|\mathrm{R}_{\mathrm{div}}^{\mathfrak{s}}\left(v^{i}\right)\right|^{2} & \leq \frac{m_{\mathfrak{s}}^{2}}{h_{\mathcal{D}}^{2}+h_{\mathcal{D}^{\prime}}^{2}}\left|v_{\mathcal{K}}^{i}-v_{\mathfrak{s}}^{i}\right|^{2}+\frac{m_{\mathfrak{s}}^{2}}{h_{\mathcal{D}}^{2}+h_{\mathcal{D}^{\prime}}^{2}}\left|v_{\mathcal{K}^{*}}^{i}-v_{\mathfrak{s}}^{i}\right|^{2} \\
& \leq C \int_{\widehat{\mathcal{K}} \cup \widehat{\mathcal{K}^{*}}}\left|\nabla v^{i}(z)\right|^{2} \mathrm{~d} z .
\end{aligned}
$$

Thus, we obtain for $i=1,2$

$$
\sum_{\mathfrak{s}=\mathcal{D} \mid \mathcal{D}^{\prime} \in \mathfrak{S}} \frac{m_{\mathfrak{s}}^{2}}{h_{\mathcal{D}}^{2}+h_{\mathcal{D}^{\prime}}^{2}}\left|\mathrm{R}_{\text {div }}^{\mathfrak{s}}\left(v^{i}\right)\right|^{2} \leq C \int_{\Omega}\left|\nabla v^{i}(z)\right|^{2} \mathrm{~d} z .
$$

Summing over $i$ gives the result.

6. Stability of the scheme. In this section, we prove the uniform stability of the DDFV scheme thanks to the right stabilization term. The stabilization already plays a role to prove the well-posedness of our scheme, in Section 4. In Theorem 6.1, the choice of the stabilization term à la Brezzi-Pitkäranta is crucial, since we get Remark 3.6. Let us first introduce the bilinear form associated to our DDFV scheme (4.1):

DEFINITION 6.1. We define the bilinear form associated to our DDFV scheme (4.1):

$$
\begin{aligned}
& \forall\left(\mathbf{u}^{\mathcal{T}}, p^{\mathfrak{D}}\right),\left(\widetilde{\mathbf{u}}^{\mathcal{T}}, \widetilde{p}^{\mathfrak{D}}\right) \in\left(\mathbb{R}^{2}\right)^{\mathcal{T}} \times \mathbb{R}^{\mathfrak{D}}, \\
& B\left(\mathbf{u}^{\mathcal{T}}, p^{\mathfrak{D}} ; \widetilde{\mathbf{u}}^{\mathcal{T}}, \widetilde{p}^{\mathfrak{D}}\right)= \llbracket \operatorname{div}^{\mathcal{T}}\left(-2 \eta^{\mathfrak{D}} \mathrm{D}^{\mathfrak{D}} \mathbf{u}^{\mathcal{T}}+p^{\mathfrak{D}} \mathrm{Id}\right), \widetilde{\mathbf{u}}^{\mathcal{T}} \rrbracket_{\mathcal{T}} \\
&+\left(\operatorname{div}^{\mathfrak{D}}\left(\mathbf{u}^{\mathcal{T}}\right)-\lambda h_{\mathfrak{D}}^{2} \Delta^{\mathfrak{D}} p^{\mathfrak{D}}, \widetilde{p}^{\mathfrak{D}}\right)_{\mathfrak{D}},
\end{aligned}
$$

where the stabilization parameter $\lambda$ is a positive number.

THEOREM 6.1 (Stability of the scheme). Assume that $\eta$ satisfies (2.3). Then there exists $C_{1}>0$ and $C_{2}>0$, depending only on the diameter of $\Omega, \lambda, \underline{\mathrm{C}}_{\eta}, \overline{\mathrm{C}}_{\eta}$ and $\operatorname{reg}(\mathcal{T})$, such that for each pair $\left(\mathbf{u}^{\mathcal{T}}, p^{\mathfrak{D}}\right) \in \mathbb{E}_{0} \times \mathbb{R}^{\mathfrak{D}}$ such that $\sum_{\mathcal{D} \in \mathfrak{D}} m_{\mathcal{D}} p^{\mathcal{D}}=0$, there exists $\widetilde{\mathbf{u}}^{\mathcal{T}} \in \mathbb{E}_{0}$ and $\widetilde{p}^{\mathfrak{D}} \in \mathbb{R}^{\mathfrak{D}}:$

$$
\left\|\nabla^{\mathfrak{D}} \widetilde{\mathbf{u}}^{\mathcal{T}}\right\|_{2}+\left\|\widetilde{p}^{\mathfrak{D}}\right\|_{2} \leq C_{1}\left(\left\|\nabla^{\mathfrak{D}} \mathbf{u}^{\mathcal{T}}\right\|_{2}+\left\|p^{\mathfrak{D}}\right\|_{2}\right)
$$


and

$$
\left\|\nabla^{\mathfrak{D}} \mathbf{u}^{\mathcal{T}}\right\|_{2}^{2}+\left\|p^{\mathfrak{D}}\right\|_{2}^{2} \leq C_{2} B\left(\mathbf{u}^{\mathcal{T}}, p^{\mathfrak{D}} ; \widetilde{\mathbf{u}}^{\mathcal{T}}, \widetilde{p}^{\mathfrak{D}}\right) .
$$

Proof. Let $\left(\mathbf{u}^{\mathcal{T}}, p^{\mathcal{D}}\right) \in \mathbb{E}_{0} \times \mathbb{R}^{\mathcal{D}}$ such that $\sum_{\mathcal{D} \in \mathfrak{D}} m_{\mathcal{D}} p^{\mathcal{D}}=0$. The proof of this Theorem is obtained by building explicitly $\left(\widetilde{\mathbf{u}}^{\mathcal{T}}, \widetilde{p}^{\mathfrak{D}}\right) \in \mathbb{E}_{0} \times \mathbb{R}^{\mathfrak{D}}$ such that the relations (6.1) and (6.2) hold.

Step 1. We apply the discrete Stokes formula Theorem 3.1 to $B$ Definition 6.1, remark that $\gamma^{\mathcal{T}}\left(\mathbf{u}^{\mathcal{T}}\right)=0$ and that $\mathrm{D}^{\mathfrak{D}} \mathbf{u}^{\mathcal{T}}$ is symmetric, we get

$$
B\left(\mathbf{u}^{\mathcal{T}}, p^{\mathfrak{P}} ; \mathbf{u}^{\mathcal{T}}, p^{\mathfrak{D}}\right)=\left(2 \eta^{\mathfrak{D}} \mathrm{D}^{\mathfrak{D}} \mathbf{u}^{\mathcal{T}}: \mathrm{D}^{\mathfrak{D}} \mathbf{u}^{\mathcal{T}}\right)_{\mathfrak{D}}-\lambda\left(h_{\mathfrak{D}}^{2} \Delta^{\mathfrak{D}} p^{\mathfrak{D}}, p^{\mathfrak{D}}\right)_{\mathfrak{D}} .
$$

Thanks to the inequality (2.3) and Remark 3.6, we obtain

$$
B\left(\mathbf{u}^{\mathcal{T}}, p^{\mathfrak{D}} ; \mathbf{u}^{\mathcal{T}}, p^{\mathfrak{D}}\right) \geq 2 \underline{\mathrm{C}}_{\eta}\left\|\mathrm{D}^{\mathfrak{D}} \mathbf{u}^{\mathcal{T}}\right\|_{2}^{2}+\lambda\left|p^{\mathfrak{D}}\right|_{h}^{2} .
$$

Finally we use the discrete Korn inequality Theorem 5.1 in order to get

$$
B\left(\mathbf{u}^{\mathcal{T}}, p^{\mathfrak{D}} ; \mathbf{u}^{\mathcal{T}}, p^{\mathfrak{D}}\right) \geq \underline{\mathrm{C}}_{\eta}\left\|\nabla^{\mathfrak{D}} \mathbf{u}^{\mathcal{T}}\right\|_{2}^{2}+\lambda\left|p^{\mathfrak{P}}\right|_{h}^{2} .
$$

Note that the above estimate on the pressure is mesh dependent. Recall that the semi-norm $|\cdot|_{h}$ is itself mesh dependent. That is why we could not bound uniformly the $L^{2}$-norm of the pressure by the semi-norm $|\cdot|_{h}$.

Step 2. We use the Nečas Lemma (see [22, Corollary 2.4] or [4, Lemma III.1.17]): since $p^{\mathfrak{D}}=\sum_{\mathcal{D} \in \mathfrak{D}} p^{\mathcal{D}} \mathbf{1}_{\mathcal{D}} \in L^{2}(\Omega)$ and its integral over $\Omega$ is zero, there exists a constant $C>0$ depending only on $\Omega$, and $\mathbf{v} \in\left(H_{0}^{1}(\Omega)\right)^{2}$ such that $\operatorname{div}(\mathbf{v})=-p^{\mathfrak{D}}$ and

$$
\|\mathbf{v}\|_{H^{1}} \leq C\left\|p^{\mathfrak{D}}\right\|_{2} \text {. }
$$

Let us choose $\mathbf{v}^{\mathcal{T}}=\mathbb{P}_{m}^{\mathcal{T}} \mathbf{v}$ the mean-value projection $\mathbb{P}_{\boldsymbol{m}}^{\mathcal{T}} \mathbf{v}$, defined by (3.5). In particular, we have $\mathbf{v}^{\mathcal{T}} \in \mathbb{E}_{0}$. Using Lemma 5.5, we obtain

$$
\left\|\nabla^{\mathfrak{D}} \mathbf{v}^{\mathcal{T}}\right\|_{2} \leq C\|\mathbf{v}\|_{H^{1}} \leq C\left\|p^{\mathfrak{D}}\right\|_{2} .
$$

The discrete Stokes formula Theorem 3.1 implies

$$
B\left(\mathbf{u}^{\mathcal{T}}, p^{\mathfrak{D}} ; \mathbf{v}^{\mathcal{T}}, 0\right)=2\left(\eta^{\mathfrak{D}} \mathrm{D}^{\mathfrak{D}} \mathbf{u}^{\mathcal{T}}: \nabla^{\mathfrak{D}} \mathbf{v}^{\mathcal{T}}\right)_{\mathfrak{D}}-\left(p^{\mathfrak{D}}, \operatorname{div}^{\mathfrak{D}}\left(\mathbf{v}^{\mathcal{T}}\right)\right)_{\mathfrak{D}} .
$$

Using the Cauchy-Schwarz inequality, adding and subtracting $\sum_{\mathcal{D} \in \mathfrak{D}} \int_{\mathcal{D}} p^{\mathcal{D}} \operatorname{div}(\mathbf{v}(z)) \mathrm{d} z$, we deduce

$$
\begin{aligned}
B\left(\mathbf{u}^{\mathcal{T}}, p^{\mathfrak{P}} ; \mathbf{v}^{\mathcal{T}}, 0\right) \geq & -\overline{\mathrm{C}}_{\eta}\left\|\mathrm{D}^{\mathcal{D}} \mathbf{u}^{\mathcal{T}}\right\|_{2}\left\|\nabla^{\mathcal{D}} \mathbf{v}^{\mathcal{T}}\right\|_{2}-\sum_{\mathcal{D} \in \mathcal{D}} \int_{\mathcal{D}} p^{\mathcal{D}} \operatorname{div}(\mathbf{v}(z)) \mathrm{d} z \\
& -\sum_{\mathcal{D} \in \mathcal{D}} \int_{\mathcal{D}} p^{\mathcal{D}}\left(\operatorname{div}^{\mathcal{D}}\left(\mathbf{v}^{\mathcal{T}}\right)-\operatorname{div}(\mathbf{v}(z))\right) \mathrm{d} z .
\end{aligned}
$$

Since we have $\operatorname{div}(\mathbf{v})=-p^{\mathfrak{P}}$, the inequality (6.5) and Proposition 5.1 give

$$
\begin{aligned}
B\left(\mathbf{u}^{\mathcal{T}}, p^{\mathfrak{D}} ; \mathbf{v}^{\mathcal{T}}, 0\right) \geq & -C\left\|\nabla^{\mathfrak{D}} \mathbf{u}^{\mathcal{T}}\right\|_{2}\left\|p^{\mathfrak{D}}\right\|_{2}+\left\|p^{\mathfrak{D}}\right\|_{2}^{2} \\
& -\sum_{\mathcal{D} \in \mathfrak{D}} \int_{\mathcal{D}} p^{\mathcal{D}}\left(\operatorname{div}^{\mathcal{D}}\left(\mathbf{v}^{\mathcal{T}}\right)-\operatorname{div}(\mathbf{v}(z))\right) \mathrm{d} z .
\end{aligned}
$$


Thanks to Proposition 5.5 and to estimate (6.4) we have

$$
\sum_{\mathcal{D} \in \mathfrak{D}} \int_{\mathcal{D}} p^{\mathcal{D}}\left(\operatorname{div}^{\mathcal{D}}\left(\mathbf{v}^{\mathcal{T}}\right)-\operatorname{div}(\mathbf{v}(z))\right) \mathrm{d} z \leq C^{\prime}\left|p^{\mathfrak{D}}\right|_{h}\|\mathbf{v}\|_{H^{1}} \leq C\left|p^{\mathfrak{D}}\right|_{h}\left\|p^{\mathfrak{D}}\right\|_{2} .
$$

We deduce that

$$
B\left(\mathbf{u}^{\mathcal{T}}, p^{\mathfrak{D}} ; \mathbf{v}^{\mathcal{T}}, 0\right) \geq\left\|p^{\mathfrak{D}}\right\|_{2}^{2}-C\left\|p^{\mathfrak{D}}\right\|_{2}\left\|\nabla^{\mathfrak{D}} \mathbf{u}^{\mathcal{T}}\right\|_{2}-C\left|p^{\mathfrak{D}}\right|_{h}\left\|p^{\mathfrak{D}}\right\|_{2}
$$

Using Young's inequality, we obtain the existence of three constants $C_{1}, C_{2}, C_{3}>0$, depending only on $\Omega, \overline{\mathrm{C}}_{\eta}$ and $\operatorname{reg}(\mathcal{T})$, such that

$$
B\left(\mathbf{u}^{\mathcal{T}}, p^{\mathfrak{D}} ; \mathbf{v}^{\mathcal{T}}, 0\right) \geq C_{1}\left\|p^{\mathfrak{D}}\right\|_{2}^{2}-C_{2}\left\|\nabla^{\mathfrak{D}} \mathbf{u}^{\mathcal{T}}\right\|_{2}^{2}-C_{3}\left|p^{\mathfrak{D}}\right|_{h}^{2}
$$

Step 3. By bilinearity of $B$, the inequalities (6.3) and (6.6) give for each positive number $\xi>0$ :

$B\left(\mathbf{u}^{\mathcal{T}}, p^{\mathfrak{D}} ; \mathbf{u}^{\mathcal{T}}+\xi \mathbf{v}^{\mathcal{T}}, p^{\mathfrak{D}}\right) \geq\left(\underline{\mathrm{C}}_{\eta}-\xi C_{2}\right)\left\|\nabla^{\mathfrak{D}} \mathbf{u}^{\mathcal{T}}\right\|_{2}^{2}+\xi C_{1}\left\|p^{\mathfrak{D}}\right\|_{2}^{2}+\left(\lambda-\xi C_{3}\right)\left|p^{\mathfrak{D}}\right|_{h}^{2}$.

Choosing a value of $\xi>0$ small enough (depending only on $\underline{\mathrm{C}}_{\eta}, C_{2}, \lambda$ and $C_{3}$ ) to have all the constant coefficients in front of the norms positive, this inequality (6.7) yields an estimate of the form (6.2). As the relation (6.1) is clearly verified by the pair $\widetilde{\mathbf{u}}^{\mathcal{T}}=\mathbf{u}^{\mathcal{T}}+\xi \mathbf{v}^{\mathcal{T}}$ and $\widetilde{p}^{\mathfrak{D}}=p^{\mathfrak{D}}$, this concludes the proof.

REMARK 6.1. It is clear that the proof breaks down if we do not consider the stabilization term (i.e. if $\lambda=0$ ).

An immediate consequence of this stability inequality is the continuous dependence of the DDFV solution with respect to the data.

COROLlary 6.1. Assume that $\eta$ satisfies (2.3). There exists $C>0$, depending only on the diameter of $\Omega, \lambda, \underline{\mathrm{C}}_{\eta}, \overline{\mathrm{C}}_{\eta}$ and $\operatorname{reg}(\mathcal{T})$, such that the couple $\left(\mathbf{u}^{\mathcal{T}}, p^{\mathfrak{D}}\right) \in \mathbb{E}_{0} \times \mathbb{R}^{\mathfrak{D}}$, solution of the scheme (4.1) with $\mathrm{g}=0$, satisfies:

$$
\left\|\nabla^{\mathfrak{D}} \mathbf{u}^{\mathcal{T}}\right\|_{2}^{2}+\left\|p^{\mathfrak{D}}\right\|_{2}^{2} \leq C\left\|\mathbf{f}^{\mathcal{T}}\right\|_{2}^{2} .
$$

Proof. Let $\left(\mathbf{u}^{\mathcal{T}}, p^{\mathfrak{D}}\right) \in \mathbb{E}_{0} \times \mathbb{R}^{\mathfrak{D}}$ solution of the scheme (4.1). Thanks to Theorem 6.1, there exists $\widetilde{\mathbf{u}}^{\mathcal{T}} \in \mathbb{E}_{0}, \widetilde{p}^{\mathfrak{D}} \in \mathbb{R}^{\mathfrak{D}}$, such that

$$
\left\|\nabla^{\mathfrak{D}} \widetilde{\mathbf{u}}^{\mathcal{T}}\right\|_{2}+\left\|\widetilde{p}^{\mathfrak{D}}\right\|_{2} \leq C_{1}\left(\left\|\nabla^{\mathfrak{D}} \mathbf{u}^{\mathcal{T}}\right\|_{2}+\left\|p^{\mathfrak{D}}\right\|_{2}\right)
$$

and

$$
\left\|\nabla^{\mathfrak{D}} \mathbf{u}^{\mathcal{T}}\right\|_{2}^{2}+\left\|p^{\mathfrak{D}}\right\|_{2}^{2} \leq C_{2} B\left(\mathbf{u}^{\mathcal{T}}, p^{\mathfrak{D}} ; \widetilde{\mathbf{u}}^{\mathcal{T}}, \widetilde{p}^{\mathfrak{D}}\right) .
$$

By Definition 6.1 of $B$, we get $B\left(\mathbf{u}^{\mathcal{T}}, p^{\mathfrak{D}} ; \widetilde{\mathbf{u}}^{\mathcal{T}}, \widetilde{p}^{\mathfrak{D}}\right)=\llbracket \mathbf{f}^{\mathcal{T}}, \widetilde{\mathbf{u}}^{\mathcal{T}} \rrbracket_{\mathcal{T}}$. Using the CauchySchwarz inequality and the discrete Poincaré inequality (Theorem 5.2), we get

$$
\left\|\nabla^{\mathfrak{D}} \mathbf{u}^{\mathcal{T}}\right\|_{2}^{2}+\left\|p^{\mathfrak{D}}\right\|_{2}^{2} \leq C_{2}\left\|\mathbf{f}^{\mathcal{T}}\right\|_{2}\left\|\widetilde{\mathbf{u}}^{\mathcal{T}}\right\|_{2} \leq C\left\|\mathbf{f}^{\mathcal{T}}\right\|_{2}\left\|\nabla^{\mathfrak{D}} \widetilde{\mathbf{u}}^{\mathcal{T}}\right\|_{2}
$$

Using (6.8) and Young inequality, the claim is proved.

7. Error estimates. We now provide an error estimate in the case when the exact solution of the problem (2.1) lies in the space $\left(H^{2}(\Omega)\right)^{2} \times H^{1}(\Omega)$ and the viscosity is smooth enough. Our main result is the following

THEOREM 7.1. Assume that $\eta$ satisfies (2.3) and (2.4). Assume that the solution $(\mathbf{u}, p)$ of the problem (2.1) belongs to $\left(H^{2}(\Omega)\right)^{2} \times H^{1}(\Omega)$. Let $\left(\mathbf{u}^{\mathcal{T}}, p^{\mathfrak{D}}\right) \in\left(\mathbb{R}^{2}\right)^{\mathcal{T}} \times \mathbb{R}^{\mathcal{D}}$ be the 
solution of the scheme (4.1). There exists a constant $C>0$ depending only on $\operatorname{reg}(\mathcal{T}), \lambda$, $\mathrm{C}_{\eta}, \underline{\mathrm{C}}_{\eta}, \overline{\mathrm{C}}_{\eta},\|\mathbf{u}\|_{H^{2}}$ and $\|p\|_{H^{1}}$, such that:

$$
\left\|\mathbf{u}-\mathbf{u}^{\mathcal{T}}\right\|_{2}+\left\|\nabla \mathbf{u}-\nabla^{\mathcal{D}} \mathbf{u}^{\mathcal{T}}\right\|_{2} \leq C \operatorname{size}(\mathcal{T}),
$$

and

$$
\left\|p-p^{\mathfrak{D}}\right\|_{2} \leq C \operatorname{size}(\mathcal{T})
$$

As usual for the error analysis of finite volume methods, the consistency error which has to be studied is the error on the numerical fluxes across each of the edges and dual edges in the mesh. Therefore consistency errors are naturally defined on the diamond cells.

DEFINITION 7.1. We define the consistency errors on $\mathfrak{D}$ for any $\mathbf{v} \in\left(H^{2}(\Omega)\right)^{2}$ and for any $p \in H^{1}(\Omega)$

$$
\begin{aligned}
& R_{\mathfrak{D}}^{\mathbf{v}}(z)=\eta(z) \operatorname{Dv}(z)-\eta_{\mathcal{D}} \mathrm{D}^{\mathcal{D}} \mathfrak{P}_{m, \gamma(\mathbf{v})} \mathbb{P}_{\boldsymbol{c}}^{\mathcal{T}} \mathbf{v}, \quad \text { for } z \in \mathcal{D}, \mathcal{D} \in \mathfrak{D}, \\
& R_{\mathfrak{D}}^{p}(z)=\mathbb{P}_{m}^{\mathcal{D}} p-p(z), \quad \text { for } z \in \mathcal{D}, \mathcal{D} \in \mathfrak{D} .
\end{aligned}
$$

Recall that the edges $\sigma$ and $\sigma^{*}$ are the diagonals of $\mathcal{D}=\mathcal{D}_{\sigma, \sigma^{*}}$. Let us introduce the following consistency errors on the numerical fluxes, for $i \in\{\mathbf{v}, p\}$ :

$$
\begin{array}{rlll}
\mathbf{R}_{\sigma, \mathcal{K}}^{i} & =-\mathbf{R}_{\sigma, \mathcal{L}}^{i}= & \frac{1}{m_{\sigma}} \int_{\sigma} R_{\mathfrak{D}}^{i}(s) \overrightarrow{\mathbf{n}}_{\sigma \mathcal{K}} \mathrm{d} s, \\
\mathbf{R}_{\sigma^{*}, \mathcal{K}^{*}}^{i} & =-\mathbf{R}_{\sigma^{*}, \mathcal{L}^{*}}^{i}= & \frac{1}{m_{\sigma^{*}}} \int_{\sigma^{*}} R_{\mathfrak{D}}^{i}(s) \overrightarrow{\mathbf{n}}_{\sigma^{*} \mathcal{K}^{*}} \mathrm{~d} s, \\
\mathbf{R}_{\sigma}^{i} & =\left|\mathbf{R}_{\sigma, \mathcal{K}}^{i}\right|= & \left|\mathbf{R}_{\sigma, \mathcal{L}}^{i}\right|, \\
\mathbf{R}_{\sigma^{*}}^{i}= & \left|\mathbf{R}_{\sigma^{*}, \mathcal{K}^{*}}^{i}\right| & = & \left|\mathbf{R}_{\sigma^{*}, \mathcal{L}^{*}}^{i}\right| .
\end{array}
$$

We note the $L^{2}$-norm of the consistency error as follows, for $i=\mathbf{v}, p$ :

$$
\left\|\mathbf{R}_{\sigma}^{i}\right\|_{2}^{2}=\sum_{\mathcal{D}_{\sigma, \sigma^{*}} \in \mathfrak{D}} m_{\mathcal{D}}\left|\mathbf{R}_{\sigma}^{i}\right|^{2}, \quad\left\|\mathbf{R}_{\sigma^{*}}^{i}\right\|_{2}^{2}=\sum_{\mathcal{D}_{\sigma, \sigma^{*}} \in \mathfrak{D}} m_{\mathcal{D}}\left|\mathbf{R}_{\sigma^{*}}^{i}\right|^{2} .
$$

7.1. First step proof of Theorem 7.1. Let $\mathbf{e}^{\mathcal{T}}=\mathfrak{P}_{m, g} \mathbb{P}_{c}^{\mathcal{T}} \mathbf{u}-\mathbf{u}^{\mathcal{T}} \in \mathbb{E}_{0}$ denote the approximation error for the velocity solution field and $e^{\mathfrak{D}}=\mathbb{P}_{m}^{\mathfrak{D}} p-p^{\mathfrak{D}} \in \mathbb{R}^{\mathfrak{D}}$ the approximation error for the pressure solution field. Recall that $\mathbf{g}=\gamma(\mathbf{u})$. Thanks to (4.1) and (2.1), we have $\forall \mathcal{K} \in \mathfrak{M}$

$$
\begin{aligned}
& \operatorname{div}^{\mathcal{K}}\left(-2 \eta^{\mathfrak{D}} \mathrm{D}^{\mathfrak{D}} \mathbf{u}^{\mathcal{T}}+p^{\mathfrak{D}} \mathrm{Id}\right)=\mathbf{f}_{\mathcal{K}}, \\
&-\frac{1}{m_{\mathcal{K}}} \int_{\mathcal{K}} \operatorname{div}(2 \eta(x) \operatorname{Du}(x)) \mathrm{d} x+\frac{1}{m_{\mathcal{K}}} \int_{\mathcal{K}} \nabla p(x) \mathrm{d} x=\mathbf{f}_{\mathcal{K}} .
\end{aligned}
$$

Therefore, we deduce

$$
\begin{aligned}
m_{\mathcal{K}} \operatorname{div}^{\mathcal{K}}\left(-2 \eta^{\mathfrak{D}} \mathrm{D}^{\mathfrak{D}} \mathbf{e}^{\mathcal{T}}+e^{\mathfrak{D}} \mathrm{Id}\right)= & m_{\mathcal{K}} \operatorname{div}^{\mathcal{K}}\left(-2 \eta^{\mathfrak{D}} \mathrm{D}^{\mathfrak{D}} \mathfrak{P}_{\boldsymbol{m}, \boldsymbol{g}} \mathbb{P}_{\boldsymbol{c}}^{\mathcal{T}} \mathbf{u}+\mathbb{P}_{m}^{\mathfrak{D}} p \mathrm{Id}\right) \\
& +\int_{\mathcal{K}} \operatorname{div}(2 \eta(x) \operatorname{Du}(x)) \mathrm{d} x-\int_{\mathcal{K}} \nabla p(x) \mathrm{d} x .
\end{aligned}
$$

Using Definition 7.1, it becomes

$$
m_{\mathcal{K}} \operatorname{div}^{\mathcal{K}}\left(-2 \eta^{\mathfrak{D}} \mathrm{D}^{\mathfrak{D}} \mathbf{e}^{\mathcal{T}}+e^{\mathfrak{D}} \mathrm{Id}\right)=2 \sum_{\mathcal{D} \in \mathfrak{D}_{\mathcal{K}}} m_{\sigma} \mathbf{R}_{\sigma, \mathcal{K}}^{\mathbf{u}}+\sum_{\mathcal{D} \in \mathfrak{D}_{\mathcal{K}}} m_{\sigma} \mathbf{R}_{\sigma, \mathcal{K}}^{p}
$$


In the same way, we have $\forall \mathcal{K}^{*} \in \mathfrak{M}^{*}$

$$
m_{\mathcal{K}^{*}}\left(\operatorname{div}^{\mathcal{K}^{*}}\left(-2 \eta^{\mathfrak{D}} \mathrm{D}^{\mathfrak{D}} \mathbf{e}^{\mathcal{T}}+e^{\mathfrak{D}} \mathrm{Id}\right)\right)=2 \sum_{\mathcal{D} \in \mathfrak{D}_{\mathcal{K}^{*}}} m_{\sigma^{*}} \mathbf{R}_{\sigma^{*}, \mathcal{K}^{*}}^{\mathbf{u}}+\sum_{\mathcal{D} \in \mathfrak{D}_{\mathcal{K}^{*}}} m_{\sigma^{*}} \mathbf{R}_{\sigma^{*}, \mathcal{K}^{*}}^{p} .
$$

Finally, the couple $\left(\mathbf{e}^{\mathcal{T}}, e^{\mathfrak{D}}\right) \in \mathbb{E}_{0} \times \mathbb{R}^{\mathfrak{D}}$ satisfies:

$$
\left\{\begin{aligned}
\operatorname{div}^{\mathfrak{M}}\left(-2 \eta^{\mathfrak{D}} \mathrm{D}^{\mathfrak{D}} \mathbf{e}^{\mathcal{T}}+e^{\mathfrak{D}} \mathrm{Id}\right) & =\mathbf{R}_{\mathfrak{M}} \\
\operatorname{div}^{\mathfrak{M}}\left(-2 \eta^{\mathfrak{D}} \mathrm{D}^{\mathfrak{D}} \mathbf{e}^{\mathcal{T}}+e^{\mathfrak{D}} \mathrm{Id}\right) & =\mathbf{R}_{\mathfrak{M}^{*}} \\
\operatorname{div}^{\mathfrak{D}}\left(\mathbf{e}^{\mathcal{T}}\right)-\lambda h_{\mathfrak{D}}^{2} \Delta^{\mathfrak{D}} e^{\mathfrak{D}} & =R_{\mathfrak{D}} \\
\sum_{\mathcal{D} \in \mathfrak{D}} m_{\mathcal{D}} e^{\mathcal{D}} & =0
\end{aligned}\right.
$$

where

$$
\begin{aligned}
& \mathbf{R}_{\mathcal{K}}=\frac{2}{m_{\mathcal{K}}} \sum_{\mathcal{D} \in \mathfrak{D}_{\mathcal{K}}} m_{\sigma} \mathbf{R}_{\sigma, \mathcal{K}}^{\mathbf{u}}+\frac{1}{m_{\mathcal{K}}} \sum_{\mathcal{D} \in \mathfrak{D}_{\mathcal{K}}} m_{\sigma} \mathbf{R}_{\sigma, \mathcal{K}}^{p}, \quad \forall \mathcal{K} \in \mathfrak{M}, \\
& \mathbf{R}_{\mathcal{K}^{*}}=\frac{2}{m_{\mathcal{K}^{*}}} \sum_{\mathcal{D} \in \mathfrak{D}_{\mathcal{K}^{*}}} m_{\sigma^{*}} \mathbf{R}_{\sigma^{*}, \mathcal{K}^{*}}^{\mathbf{u}}+\frac{1}{m_{\mathcal{K}^{*}}} \sum_{\mathcal{D} \in \mathfrak{D}_{\mathcal{K}^{*}}} m_{\sigma^{*}} \mathbf{R}_{\sigma^{*}, \mathcal{K}^{*}}^{p}, \quad \forall \mathcal{K}^{*} \in \mathfrak{M}^{*} \\
& R_{\mathcal{D}}=\operatorname{div}^{\mathcal{D}}\left(\mathfrak{P}_{\boldsymbol{m}, \boldsymbol{g}^{\boldsymbol{c}}} \mathbb{P}_{\boldsymbol{c}}^{\mathcal{T}} \mathbf{u}\right)-\lambda h_{\mathcal{D}}^{2} \Delta^{\mathcal{D}} \mathbb{P}_{m}^{\mathfrak{D}} p, \quad \forall \mathcal{D} \in \mathfrak{D} .
\end{aligned}
$$

Remark that we have $\sum_{\mathcal{D} \in \mathfrak{D}} m_{\mathcal{D}} R_{\mathcal{D}}=0$. Theorem 6.1 implies that there exists $\widetilde{\mathbf{e}}^{\mathcal{T}} \in \mathbb{E}_{0}$, $\widetilde{e}^{\mathfrak{D}} \in \mathbb{R}^{\mathfrak{D}}$ and $C>0$ such that :

$$
\left\|\nabla^{\mathfrak{D}} \widetilde{\mathbf{e}}^{\mathcal{T}}\right\|_{2}+\left\|\widetilde{e}^{\mathfrak{D}}\right\|_{2} \leq C\left(\left\|\nabla^{\mathfrak{D}} \mathbf{e}^{\mathcal{T}}\right\|_{2}+\left\|e^{\mathfrak{D}}\right\|_{2}\right)
$$

and

$$
\left\|\nabla^{\mathfrak{D}} \mathbf{e}^{\mathcal{T}}\right\|_{2}^{2}+\left\|e^{\mathfrak{D}}\right\|_{2}^{2} \leq C B\left(\mathbf{e}^{\mathcal{T}}, e^{\mathfrak{D}} ; \widetilde{\mathbf{e}}^{\mathcal{T}}, \widetilde{e}^{\mathfrak{D}}\right) .
$$

Thanks to Definition 6.1 of $B$ and to $(7.1)$, we have $B\left(\mathbf{e}^{\mathcal{T}}, e^{\mathfrak{D}} ; \widetilde{\mathbf{e}}^{\mathcal{T}}, \widetilde{e}^{\mathfrak{D}}\right)=\llbracket \mathbf{R}^{\mathcal{T}}, \widetilde{\mathbf{e}}^{\mathcal{T}} \rrbracket_{\mathcal{T}}+$ $\left(R^{\mathfrak{D}}, \widetilde{e}^{\mathfrak{D}}\right)_{\mathfrak{D}}$. We note $I:=\llbracket \mathbf{R}^{\mathcal{T}}, \widetilde{\mathbf{e}}^{\mathcal{T}} \rrbracket_{\mathcal{T}}$ and $T:=\left(R^{\mathfrak{D}}, \widetilde{e}^{\mathfrak{D}}\right)_{\mathfrak{D}}$.

Estimate of $I$. Using the fact that $\widetilde{\mathbf{e}}_{\mathcal{K}^{*}}=0$ for any $\mathcal{K}^{*} \in \partial \mathfrak{M}^{*}$ and the definition of $I$, we have

$$
\begin{aligned}
I= & \frac{1}{2} \sum_{\mathcal{K} \in \mathfrak{M}} \sum_{\mathcal{D} \in \mathfrak{D}_{\mathcal{K}}} m_{\sigma}\left(\mathbf{R}_{\sigma, \mathcal{K}}^{p}+2 \mathbf{R}_{\sigma, \mathcal{K}}^{\mathbf{u}}\right) \cdot \widetilde{\mathbf{e}}_{\mathcal{K}} \\
& +\frac{1}{2} \sum_{\mathcal{K}^{*} \in \mathfrak{M}^{*} \cup \partial \mathfrak{M}^{*}} \sum_{\mathcal{D} \in \mathfrak{D}_{\mathcal{K}^{*}}} m_{\sigma}\left(\mathbf{R}_{\sigma^{*}, \mathcal{K}^{*}}^{p}+2 \mathbf{R}_{\sigma^{*}, \mathcal{K}^{*}}^{\mathbf{u}}\right) \cdot \widetilde{\mathbf{e}}_{\mathcal{K}^{*}}
\end{aligned}
$$

Reordering the summation over the set of diamond cells and using the fact that $\mathbf{R}_{\sigma, \mathcal{K}}^{i}=$ $-R_{\sigma, \mathcal{L}}^{i}$ and $\mathbf{R}_{\sigma^{*}, \mathcal{K}^{*}}^{i}=-R_{\sigma^{*}, \mathcal{L}^{*}}^{i}$ for $i=\mathbf{u}, p$, we deduce

$$
\begin{aligned}
I= & \frac{1}{2} \sum_{\mathcal{D}_{\sigma, \sigma^{*}} \in \mathfrak{D}} m_{\sigma}\left(\mathbf{R}_{\sigma, \mathcal{K}}^{p}+2 \mathbf{R}_{\sigma, \mathcal{K}}^{\mathbf{u}}\right) \cdot\left(\widetilde{\mathbf{e}}_{\mathcal{K}}-\widetilde{\mathbf{e}}_{\mathcal{L}}\right) \\
& +\frac{1}{2} \sum_{\mathcal{D}_{\sigma, \sigma^{*}} \in \mathcal{D}} m_{\sigma^{*}}\left(\mathbf{R}_{\sigma^{*}, \mathcal{K}^{*}}^{p}+2 \mathbf{R}_{\sigma^{*}, \mathcal{K}^{*}}^{\mathbf{u}}\right) \cdot\left(\widetilde{\mathbf{e}}_{\mathcal{K}^{*}}-\widetilde{\mathbf{e}}_{\mathcal{L}^{*}}\right) .
\end{aligned}
$$


Using Definition 3.1 of the discrete gradient and the Cauchy-Schwarz inequality, we obtain

$$
\begin{aligned}
I= & -\sum_{\mathcal{D}_{\sigma, \sigma^{*}} \in \mathcal{D}} \frac{m_{\mathcal{D}}}{\sin \left(\alpha_{\mathcal{D}}\right)}\left(\mathbf{R}_{\sigma, \mathcal{K}}^{p}+2 \mathbf{R}_{\sigma, \mathcal{K}}^{\mathbf{u}}\right) \cdot\left(\left(\nabla^{\mathcal{D}} \widetilde{\mathbf{e}}^{\mathcal{T}}\right) \overrightarrow{\boldsymbol{\tau}}_{\mathcal{K}, \mathcal{L}}\right) \\
& -\sum_{\mathcal{D}_{\sigma, \sigma^{*}} \in \mathcal{D}} \frac{m_{\mathcal{D}}}{\sin \left(\alpha_{\mathcal{D}}\right)}\left(\mathbf{R}_{\sigma^{*}, \mathcal{K}^{*}}^{p}+2 \mathbf{R}_{\sigma^{*}, \mathcal{K}^{*}}^{\mathbf{u}}\right) \cdot\left(\left(\nabla^{\mathcal{D}} \widetilde{\mathbf{e}}^{\mathcal{T}}\right) \overrightarrow{\boldsymbol{\tau}}_{\mathcal{K}^{*}, \mathcal{L}^{*}}\right) \\
\leq & \left\|\nabla^{\mathfrak{D}} \widetilde{\mathbf{e}}^{\mathcal{T}}\right\|_{2} \frac{C}{\sin \left(\alpha_{\mathcal{T}}\right)}\left[\left\|\mathbf{R}_{\sigma}^{\mathbf{u}}\right\|_{2}+\left\|\mathbf{R}_{\sigma^{*}}^{\mathbf{u}}\right\|_{2}+\left\|\mathbf{R}_{\sigma}^{p}\right\|_{2}+\left\|\mathbf{R}_{\sigma^{*}}^{p}\right\|_{2}\right] .
\end{aligned}
$$

Estimate of $T$. First, Corollary 5.2 and Lemma 5.4 imply

$$
\left\|\operatorname{div}^{\mathfrak{D}}\left(\mathfrak{P}_{\boldsymbol{m}, \boldsymbol{g}} \mathbb{P}_{\boldsymbol{c}}^{\mathcal{T}} \mathbf{u}\right)\right\|_{2} \leq C \operatorname{size}(\mathcal{T})\|\mathbf{u}\|_{H^{2}} .
$$

Applying the Cauchy-Schwarz inequality to $\left(\operatorname{div}^{\mathfrak{D}}\left(\mathfrak{P}_{m, g} \mathbb{P}_{c}^{\mathcal{T}} \mathbf{u}\right), \widetilde{e}^{\mathfrak{D}}\right)_{\mathfrak{D}}$, we get

$$
\left(\operatorname{div}^{\mathfrak{D}}\left(\mathfrak{P}_{\boldsymbol{m}, \boldsymbol{g}} \mathbb{P}_{\boldsymbol{c}}^{\mathcal{T}} \mathbf{u}\right), \widetilde{e}^{\mathfrak{D}}\right)_{\mathfrak{D}} \leq C \operatorname{size}(\mathcal{T})\|\mathbf{u}\|_{H^{2}}\left\|\widetilde{e}^{\mathfrak{D}}\right\|_{2} .
$$

Reordering the summation over $\mathfrak{s} \in \mathfrak{S}$ in the term $T_{1}:=-\left(\lambda h_{\mathfrak{D}}^{2} \Delta^{\mathfrak{D}} \mathbb{P}_{m}^{\mathfrak{D}} p, \widetilde{e}^{\mathfrak{D}}\right)_{\mathfrak{D}}$, we have as in Remark 3.6

$$
T_{1}=-\lambda \sum_{\mathcal{D} \in \mathfrak{D}} m_{\mathcal{D}} \widetilde{e}^{\mathcal{D}} h_{\mathcal{D}}^{2} \Delta^{\mathcal{D}} \mathbb{P}_{m}^{\mathfrak{D}} p=\lambda \sum_{\mathfrak{s}=\mathcal{D} \mid \mathcal{D}^{\prime} \in \mathfrak{S}}\left(h_{\mathcal{D}}^{2}+h_{\mathcal{D}^{\prime}}^{2}\right)\left(\mathbb{P}_{m}^{\mathcal{D}^{\prime}} p-\mathbb{P}_{m}^{\mathcal{D}} p\right)\left(\widetilde{e}^{\mathcal{D}^{\prime}}-\widetilde{e}^{\mathcal{D}}\right) .
$$

The Cauchy-Schwarz inequality and (3.10) imply:

$$
\begin{aligned}
\left|T_{1}\right| & \leq \lambda\left(\sum_{\mathfrak{s}=\mathcal{D} \mid \mathcal{D}^{\prime} \in \mathfrak{S}}\left(h_{\mathcal{D}}^{2}+h_{\mathcal{D}^{\prime}}^{2}\right)\left(\mathbb{P}_{m}^{\mathcal{D}^{\prime}} p-\mathbb{P}_{m}^{\mathcal{D}} p\right)^{2}\right)^{\frac{1}{2}}\left(\sum_{\mathfrak{s}=\mathcal{D} \mid \mathcal{D}^{\prime} \in \mathfrak{S}}\left(h_{\mathcal{D}}^{2}+h_{\mathcal{D}^{\prime}}^{2}\right)\left(\widetilde{e}^{\mathcal{D}^{\prime}}-\widetilde{e}^{\mathcal{D}}\right)^{2}\right)^{\frac{1}{2}} \\
& \leq 2 \operatorname{size}(\mathcal{T}) \lambda\left|\widetilde{e}^{\mathfrak{D}}\right|_{h}\left(\sum_{\mathfrak{s}=\mathcal{D} \mid \mathcal{D}^{\prime} \in \mathfrak{S}}\left(\mathbb{P}_{m}^{\mathcal{D}^{\prime}} p-\mathbb{P}_{m}^{\mathcal{D}} p\right)^{2}\right)^{\frac{1}{2}} .
\end{aligned}
$$

Using Lemmas 3.1 and 5.7, we obtain

$$
\left|T_{1}\right| \leq C \operatorname{size}(\mathcal{T})\left\|\widetilde{e}^{\mathcal{D}}\right\|_{2}\|\nabla p\|_{2}
$$

Remark that $T=\left(\operatorname{div}^{\mathfrak{D}}\left(\mathfrak{P}_{\boldsymbol{m}, \boldsymbol{g}} \mathbb{P}_{\boldsymbol{c}}^{\mathcal{T}} \mathbf{u}\right), \widetilde{e}^{\mathfrak{D}}\right)_{\mathfrak{D}}+T_{1}$, thanks to (7.4) and (7.5), we deduce

$$
|T| \leq C \operatorname{size}(\mathcal{T})\left\|\widetilde{e}^{\mathfrak{D}}\right\|_{2}\left(\|\mathbf{u}\|_{H^{2}}+\|\nabla p\|_{2}\right) .
$$

Estimate of $B$. To sum up, (7.3) becomes

$$
\begin{aligned}
\left\|\nabla^{\mathfrak{D}} \mathbf{e}^{\mathcal{T}}\right\|_{2}^{2}+\left\|e^{\mathfrak{D}}\right\|_{2}^{2} \leq & \frac{C}{\sin \left(\alpha_{\mathcal{T}}\right)}\left\|\nabla^{\mathfrak{D}} \widetilde{\mathbf{e}}^{\mathcal{T}}\right\|_{2}\left[\left\|\mathbf{R}_{\sigma}^{\mathbf{u}}\right\|_{2}+\left\|\mathbf{R}_{\sigma^{*}}^{\mathbf{u}}\right\|_{2}+\left\|\mathbf{R}_{\sigma}^{p}\right\|_{2}+\left\|\mathbf{R}_{\sigma^{*}}^{p}\right\|_{2}\right] \\
& +C \operatorname{size}(\mathcal{T})\left\|\widetilde{e}^{\mathfrak{D}}\right\|_{2}\left(\|\mathbf{u}\|_{H^{2}}+\|\nabla p\|_{2}\right) .
\end{aligned}
$$

Finally, using (7.2), we deduce

$$
\begin{aligned}
\left\|\nabla^{\mathfrak{D}} \mathbf{e}^{\mathcal{T}}\right\|_{2}^{2}+\left\|e^{\mathfrak{D}}\right\|_{2}^{2} \leq & C\left(\left\|\mathbf{R}_{\sigma}^{\mathbf{u}}\right\|_{2}+\left\|\mathbf{R}_{\sigma^{*}}^{\mathbf{u}}\right\|_{2}+\left\|\mathbf{R}_{\sigma}^{p}\right\|_{2}+\left\|\mathbf{R}_{\sigma^{*}}^{p}\right\|_{2}\right)\left(\left\|\nabla^{\mathfrak{D}} \mathbf{e}^{\mathcal{T}}\right\|_{2}+\left\|e^{\mathfrak{D}}\right\|_{2}\right) \\
& +C \operatorname{size}(\mathcal{T})\left(\|\mathbf{u}\|_{H^{2}}+\|\nabla p\|_{2}\right)\left(\left\|\nabla^{\mathfrak{D}} \mathbf{e}^{\mathcal{T}}\right\|_{2}+\left\|e^{\mathfrak{D}}\right\|_{2}\right) .
\end{aligned}
$$

It remains to estimate the consistency errors. 


\subsection{Analysis of the consistency error.}

7.2.1. Estimate of $\mathbf{R}_{\sigma}^{\mathbf{v}}$. The consistency error $R_{\mathfrak{D}}^{\mathbf{v}}$ can be split into four different contributions $R_{\mathfrak{D}}^{\mathbf{v}, \eta}, R_{\mathfrak{D}}^{\mathbf{v}, D \mathbf{v}}, R_{\mathfrak{D}}^{\mathbf{v}, z}$ and $R_{\mathfrak{D}}^{\mathbf{v}, b d}$ coming, respectively, from the errors due to the approximation with respect to the space variable of the flux $\eta(.) \operatorname{Dv}($.$) , to the approximation$ of the gradient, to the approximation of the viscosity and to the discretization of the boundary data:

$$
R_{\mathfrak{D}}^{\mathbf{v}}(z)=R_{\mathfrak{D}}^{\mathbf{v}, \eta}(z)+R_{\mathfrak{D}}^{\mathbf{v}, D \mathbf{v}}+R_{\mathfrak{D}}^{\mathbf{v}, z}+R_{\mathfrak{D}}^{\mathbf{v}, b d},
$$

where, for $z \in \mathcal{D}$,

$$
\begin{aligned}
R_{\mathfrak{D}}^{\mathbf{v}, \eta}(z) & =\eta(z) \operatorname{Dv}(z)-\frac{1}{m_{\mathcal{D}}} \int_{\mathcal{D}} \eta(x) \operatorname{Dv}(x) \mathrm{d} x, \\
R_{\mathfrak{D}}^{\mathbf{v}, \mathrm{D} \mathbf{v}} & =\frac{1}{m_{\mathcal{D}}} \int_{\mathcal{D}} \eta(x)\left(\operatorname{Dv}(x)-\mathrm{D}^{\mathcal{D}} \mathbb{P}_{\boldsymbol{c}}^{\mathcal{T}} \mathbf{v}\right) \mathrm{d} x, \\
R_{\mathfrak{D}}^{\mathbf{v}, z} & =\left(\frac{1}{m_{\mathcal{D}}} \int_{\mathcal{D}} \eta(x) \mathrm{d} x-\eta_{\mathcal{D}}\right) \mathrm{D}^{\mathcal{D}} \mathbb{P}_{\boldsymbol{c}}^{\mathcal{T}} \mathbf{v}, \\
R_{\mathfrak{D}}^{\mathbf{v}, b d} & =\eta_{\mathcal{D}}\left(\mathrm{D}^{\mathcal{D}} \mathbb{P}_{\boldsymbol{c}}^{\mathcal{T}} \mathbf{v}-\mathrm{D}^{\mathcal{D}} \mathfrak{P}_{\boldsymbol{m}, \boldsymbol{\gamma}(\mathbf{v}} \mathbb{P}_{\boldsymbol{c}}^{\mathcal{T}} \mathbf{v}\right) .
\end{aligned}
$$

In order to control $\mathbf{R}_{\sigma}^{\mathbf{v}}$ and $\mathbf{R}_{\sigma^{*}}^{\mathbf{v}}$, let us estimate separately the different terms in the right-hand side of (7.7).

Proposition 7.1 (Error due to the discrete gradient). Assume that $\eta$ satisfies (2.3). For any mesh $\mathcal{T}$ on $\Omega$, there exists a constant $C>0$, depending only on $\operatorname{reg}(\mathcal{T})$ and $\overline{\mathrm{C}}_{\eta}$, such that for any function $\mathbf{v}$ in $\left(H^{2}(\Omega)\right)^{2}$, we have

$$
\left\|R_{\mathfrak{D}}^{\mathbf{v}, \mathrm{Dv}}\right\|_{2} \leq C \operatorname{size}(\mathcal{T})\|\nabla \mathbf{v}\|_{H^{1}} .
$$

Proof. Using Jensen inequality and the inequality (2.3) we get

$$
\left\|R_{\mathfrak{D}}^{\mathbf{v}, \mathrm{Dv}}\right\|_{2}^{2} \leq \sum_{\mathcal{D} \in \mathfrak{D}} \int_{\mathcal{D}}\left(\eta(x)\left(\operatorname{Dv}(x)-\mathrm{D}^{\mathcal{D}} \mathbb{P}_{\boldsymbol{c}}^{\mathcal{T}} \mathbf{v}\right)\right)^{2} \mathrm{~d} x \leq \overline{\mathrm{C}}_{\eta}^{2}\left\|\mathrm{D} \mathbf{v}-\mathrm{D}^{\mathfrak{D}} \mathbb{P}_{\boldsymbol{c}}^{\boldsymbol{\mathcal { T }}} \mathbf{v}\right\|_{2}^{2}
$$

Remark 2.1 implies that

$$
\left\|\mathrm{D} \mathbf{v}-\mathrm{D}^{\mathfrak{D}} \mathbb{P}_{\boldsymbol{c}}^{\mathcal{T}} \mathbf{v}\right\|_{2} \leq\left\|\nabla \mathbf{v}-\nabla^{\mathfrak{D}} \mathbb{P}_{\boldsymbol{c}}^{\mathcal{T}} \mathbf{v}\right\|_{2},
$$

and then, applying Lemma 5.3, we get the result.

PROPOSITION 7.2 (Error due to the viscosity variation). Assume that $\eta$ satisfies (2.4). For any mesh $\mathcal{T}$ on $\Omega$, there exists a constant $C>0$, depending only on $\operatorname{reg}(\mathcal{T})$ and $\mathrm{C}_{\eta}$, such that for any function $\mathbf{v}$ in $\left(H^{2}(\Omega)\right)^{2}$, we have

$$
\left\|R_{\mathfrak{D}}^{\mathbf{v}, z}\right\|_{2} \leq C \operatorname{size}(\mathcal{T})\|\nabla \mathbf{v}\|_{H^{1}} .
$$

Proof. The Jensen inequality gives

$$
\left\|R_{\mathfrak{D}}^{\mathbf{v}, z}\right\|_{2}^{2} \leq \sum_{\mathcal{D} \in \mathfrak{D}} m_{\mathcal{D}} \frac{1}{m_{\mathcal{D}}}\left(\int_{\mathcal{D}} \int_{\overline{\mathcal{D}}}|\eta(x)-\eta(z)|^{2} \mathrm{~d} \mu_{\overline{\mathcal{D}}}(z) \mathrm{d} x\right)\left\|\mathrm{D}^{\mathcal{D}} \mathbb{P}_{\boldsymbol{c}}^{\mathcal{T}} \mathbf{v}\right\|_{\mathcal{F}}^{2}
$$

Thanks to (2.4) and Proposition 5.1 we have

$$
\left\|R_{\mathfrak{D}}^{\mathbf{v}, z}\right\|_{2}^{2} \leq \mathrm{C}_{\eta}^{2} \sum_{\mathcal{D} \in \mathfrak{D}}\left(\int_{\mathcal{D}} \int_{\overline{\mathcal{D}}}|x-z|^{2} \mathrm{~d} \mu_{\overline{\mathcal{D}}}(z) \mathrm{d} x\right)\left\|\mathrm{D}^{\mathcal{D}} \mathbb{P}_{\boldsymbol{c}}^{\mathcal{T}} \mathbf{v}\right\|_{\mathcal{F}}^{2} \leq C \operatorname{size}(\mathcal{T})^{2}\left\|\nabla^{\mathfrak{D}} \mathbb{P}_{\boldsymbol{c}}^{\mathcal{T}} \mathbf{v}\right\|_{2}^{2} .
$$


Finally, Corollary 5.1 gives the result.

Proposition 7.3 (Error due to the boundary data). Assume that $\eta$ satisfies (2.3). For any mesh $\mathcal{T}$ on $\Omega$, there exists a constant $C>0$, depending only on $\operatorname{reg}(\mathcal{T})$ and $\overline{\mathrm{C}}_{\eta}$, such that for any function $\mathbf{v}$ in $\left(H^{2}(\Omega)\right)^{2}$, whose trace is denoted by $\mathbf{g}=\gamma(\mathbf{v})$, we have

$$
\left\|R_{\mathfrak{D}}^{\mathbf{v}, b d}\right\|_{2} \leq C \operatorname{size}(\mathcal{T})\|\mathbf{v}\|_{H^{2}} .
$$

Proof. Inequality (2.3) and Proposition 5.1 imply

$$
\left\|R_{\mathfrak{D}}^{\mathbf{v}, b d}\right\|_{2}^{2} \leq \overline{\mathrm{C}}_{\eta}^{2}\left\|\mathrm{D}^{\mathfrak{D}}\left(\mathbb{P}_{\boldsymbol{c}}^{\mathcal{T}} \mathbf{v}-\mathfrak{P}_{\boldsymbol{m}, \boldsymbol{g}} \mathbb{P}_{\boldsymbol{c}}^{\mathcal{T}} \mathbf{v}\right)\right\|_{2}^{2} \leq C\left\|\nabla^{\mathfrak{D}}\left(\mathbb{P}_{\boldsymbol{c}}^{\mathcal{T}} \mathbf{v}-\mathfrak{P}_{\boldsymbol{m}, \boldsymbol{g}} \mathbb{P}_{\boldsymbol{c}}^{\mathcal{T}} \mathbf{v}\right)\right\|_{2}^{2},
$$

and finally, Lemma 5.4 gives the result.

PROPOSITION 7.4 (Error due to the approximate flux). Assume that $\eta$ satisfies (2.3) and (2.4). For any mesh $\mathcal{T}$ on $\Omega$, there exists a constant $C>0$, depending only on $\operatorname{reg}(\mathcal{T}), \mathrm{C}_{\eta}$ and $\overline{\mathrm{C}}_{\eta}$, such that for any function $\mathrm{v}$ in $\left(H^{2}(\Omega)\right)^{2}$, we have

$$
\sum_{\mathcal{D}_{\sigma, \sigma^{*}} \in \mathfrak{D}} m_{\mathcal{D}}\left[\frac{1}{m_{\sigma}} \int_{\sigma}\left\|R_{\mathfrak{D}}^{\mathbf{v}, \eta}(s)\right\|_{\mathcal{F}}^{2} \mathrm{~d} s+\frac{1}{m_{\sigma^{*}}} \int_{\sigma^{*}}\left\|R_{\mathfrak{D}}^{\mathbf{v}, \eta}(s)\right\|_{\mathcal{F}}^{2} \mathrm{~d} s\right] \leq C \operatorname{size}(\mathcal{T})^{2}\|\nabla \mathbf{v}\|_{H^{1}}^{2} .
$$

Proof. We apply the Jensen inequality

$$
\left\|R_{\mathfrak{D}}^{\mathbf{v}, \eta}(s)\right\|_{\mathcal{F}}^{2} \leq \frac{1}{m_{\mathcal{D}}} \int_{\mathcal{D}}\|\eta(s) \operatorname{Dv}(s)-\eta(x) \operatorname{Dv}(x)\|_{\mathcal{F}}^{2} \mathrm{~d} x .
$$

Thus, adding and subtracting $\eta(s) \operatorname{Dv}(x)$, Cauchy-Schwarz inequality implies

$$
\begin{aligned}
& \left\|R_{\mathfrak{D}}^{\mathbf{v}, \eta}(s)\right\|_{\mathcal{F}}^{2} \\
& \quad \leq \frac{2}{m_{\mathcal{D}}} \int_{\mathcal{D}}|\eta(s)-\eta(x)|^{2}\|\operatorname{Dv}(x)\|_{\mathcal{F}}^{2} \mathrm{~d} x+\frac{2}{m_{\mathcal{D}}} \int_{\mathcal{D}}|\eta(s)|^{2}\|\operatorname{Dv}(s)-\operatorname{Dv}(x)\|_{\mathcal{F}}^{2} \mathrm{~d} x .
\end{aligned}
$$

Inequalities (2.3) and (2.4) imply

$$
\left\|R_{\mathfrak{D}}^{\mathbf{v}, \eta}(s)\right\|_{\mathcal{F}}^{2} \leq \operatorname{size}(\mathcal{T})^{2} \frac{2 \mathrm{C}_{\eta}^{2}}{m_{\mathcal{D}}} \int_{\mathcal{D}}\|\operatorname{Dv}(x)\|_{\mathcal{F}}^{2} \mathrm{~d} x+\frac{2 \overline{\mathrm{C}}_{\eta}^{2}}{m_{\mathcal{D}}} \int_{\mathcal{D}}\|\operatorname{Dv}(s)-\operatorname{Dv}(x)\|_{\mathcal{F}}^{2} \mathrm{~d} x .
$$

We average the second integral on the right-hand side of (7.8) on $\sigma$ and apply Lemma 5.1

$$
\frac{1}{m_{\mathcal{D}} m_{\sigma}} \int_{\sigma} \int_{\mathcal{D}}\|\operatorname{Dv}(s)-\operatorname{Dv}(x)\|_{\mathcal{F}}^{2} \mathrm{~d} x \mathrm{~d} s \leq C \frac{h_{\mathcal{D}}^{3}}{m_{\sigma} m_{\mathcal{D}}} \int_{\mathcal{D}} \mid \nabla\left(\left.\operatorname{Dv}(y)\right|^{2} \mathrm{~d} y .\right.
$$

Note that we use here an extension of Lemma 5.1 to the matrix framework. Thanks to the relation (3.2), it follows

$$
\sum_{\mathcal{D}_{\sigma, \sigma^{*}} \in \mathfrak{D}} m_{\mathcal{D}} \frac{1}{m_{\mathcal{D}} m_{\sigma}} \int_{\sigma} \int_{\mathcal{D}}\|\mathrm{D} \mathbf{v}(s)-\operatorname{Dv}(x)\|_{\mathcal{F}}^{2} \mathrm{~d} x \mathrm{~d} s \leq C \operatorname{size}(\mathcal{T})^{2}\|\nabla \mathbf{v}\|_{H^{1}}^{2} .
$$

As a result, we obtain

$$
\sum_{\mathcal{D}_{\sigma, \sigma^{*}} \in \mathfrak{D}} m_{\mathcal{D}} \frac{1}{m_{\sigma}} \int_{\sigma}\left\|R_{\mathfrak{D}}^{\mathbf{v}, \eta}(s)\right\|_{\mathcal{F}}^{2} \mathrm{~d} s \leq C \operatorname{size}(\mathcal{T})^{2}\|\nabla \mathbf{v}\|_{H^{1}}^{2}
$$


We use the same computation for the similar term on the dual edge $\sigma^{*}$.

Now, we can control $\mathbf{R}_{\sigma}^{\mathbf{v}}$ and $\mathbf{R}_{\sigma^{*}}^{\mathbf{v}}$, as follows

COROllary 7.1. Assume that $\eta$ satisfies (2.3) and (2.4). For any mesh $\mathcal{T}$ on $\Omega$, there exists a constant $C>0$, depending only on $\operatorname{reg}(\mathcal{T}), \mathrm{C}_{\eta}$ and $\overline{\mathrm{C}}_{\eta}$, such that for any function $\mathbf{v}$ in $\left(H^{2}(\Omega)\right)^{2}$, we have

$$
\left\|\mathbf{R}_{\sigma}^{\mathbf{v}}\right\|_{2}+\left\|\mathbf{R}_{\sigma^{*}}^{\mathbf{v}}\right\|_{2} \leq C \operatorname{size}(\mathcal{T})\|\nabla \mathbf{v}\|_{H^{1}}
$$

Proof. Thanks to (7.7) and Cauchy-Schwarz inequality, we get

$$
\begin{aligned}
\sum_{\mathcal{D}_{\sigma, \sigma *} \in \mathfrak{D}} m_{\mathcal{D}}\left|\mathbf{R}_{\sigma}^{\mathbf{v}}\right|^{2} \leq & 4 \sum_{\mathcal{D}_{\sigma, \sigma^{*}} \in \mathfrak{D}} m_{\mathcal{D}} \frac{1}{m_{\sigma}} \int_{\sigma}\left\|R_{\mathfrak{D}}^{\mathbf{v}, \eta}(s)\right\|_{\mathcal{F}}^{2} \mathrm{~d} s+4\left\|R_{\mathfrak{D}}^{\mathbf{v}, \mathrm{Dv}}\right\|_{2}^{2} \\
& +4\left\|R_{\mathfrak{D}}^{\mathbf{v}, z}\right\|_{2}^{2}+4\left\|R_{\mathfrak{D}}^{\mathbf{v}, b d}\right\|_{2}^{2} .
\end{aligned}
$$

We conclude using Propositions 7.1, 7.2, 7.3 and 7.4. We proceed in the same way for the estimate of $\mathbf{R}_{\sigma^{*}}^{\mathbf{v}}$.

7.2.2. Estimate of $\mathbf{R}_{\sigma}^{p}$. Now, we can control $\mathbf{R}_{\sigma}^{p}$ and $\mathbf{R}_{\sigma^{*}}^{p}$, as follows

COROLLARY 7.2. For any mesh $\mathcal{T}$ on $\Omega$, there exists a constant $C>0$, depending only on $\operatorname{reg}(\mathcal{T})$, such that for any function $p$ in $H^{1}(\Omega)$, we have

$$
\left\|\mathbf{R}_{\sigma}^{p}\right\|_{2}+\left\|\mathbf{R}_{\sigma^{*}}^{p}\right\|_{2} \leq C \operatorname{size}(\mathcal{T})\|\nabla p\|_{2} .
$$

Proof. We use Lemma 5.2 on a primal edge $\sigma$ and a half diamond cell $\mathcal{D}_{1}$ such that $\mathcal{D}_{\sigma, \sigma^{*}}=\mathcal{D}_{1} \cup \mathcal{D}_{2}$ with $\mathcal{D}_{1} \cap \mathcal{D}_{2}=\sigma$ for $R_{\mathfrak{D}}^{p}(s) \overrightarrow{\mathbf{n}}_{\sigma \mathcal{K}}:$ for $i=1,2$

$$
\begin{aligned}
\left|\mathbf{R}_{\sigma}^{p}\right|^{2} & =\left|\frac{1}{m_{\sigma}} \int_{\sigma} R_{\mathfrak{D}}^{p}(s) \overrightarrow{\mathbf{n}}_{\sigma \mathcal{K}} \mathrm{d} s\right|^{2} \\
& \leq \frac{C h_{\mathcal{D}_{i}}}{m_{\sigma}} \int_{\mathcal{D}_{i}}\left\|\nabla\left(R_{\mathfrak{D}}^{p}(z) \overrightarrow{\mathbf{n}}_{\sigma \mathcal{K}}\right)\right\|_{\mathcal{F}}^{2} \mathrm{~d} s+\frac{C}{h_{\mathcal{D}_{i}} m_{\sigma}} \int_{\mathcal{D}_{i}}\left|\left(R_{\mathfrak{D}}^{p}(z) \overrightarrow{\mathbf{n}}_{\sigma \mathcal{K}}\right)\right|^{2} \mathrm{~d} z
\end{aligned}
$$

Thanks to the relation (3.2) we have $\frac{m_{\mathcal{D}} h_{\mathcal{D}_{i}}}{m_{\sigma}} \leq \frac{1}{2} \operatorname{size}(\mathcal{T})^{2}$ and $\frac{m_{\mathcal{D}}}{h_{\mathcal{D}_{i}} m_{\sigma}} \leq C$. Recall that $R_{\mathfrak{D}}^{p}=\mathbb{P}_{m}^{\mathcal{D}} p-p$, we deduce

$$
\sum_{\mathcal{D}_{\sigma, \sigma^{*}} \in \mathfrak{D}} m_{\mathcal{D}}\left|\mathbf{R}_{\sigma}^{p}\right|^{2} \leq C \operatorname{size}(\mathcal{T})^{2} \sum_{\mathcal{D} \in \mathfrak{D}} \int_{\mathcal{D}}|\nabla p(z)|^{2} \mathrm{~d} z+C \sum_{\mathcal{D} \in \mathfrak{D}} \int_{\mathcal{D}}\left|\mathbb{P}_{m}^{\mathcal{D}} p-p(z)\right|^{2} \mathrm{~d} z .
$$

Finally, Proposition 5.4 gives

$$
\sum_{\mathcal{D}_{\sigma, \sigma^{*}} \in \mathfrak{D}} m_{\mathcal{D}}\left|\mathbf{R}_{\sigma}^{p}\right|^{2} \leq C \operatorname{size}(\mathcal{T})^{2}\|\nabla p\|_{2}^{2}
$$

We proceed in the same way for the estimate of $\mathbf{R}_{\sigma^{*}}^{p}$.

7.3. Concluding the proof of Theorem 7.1. We may now collect all the previous results in order to conclude the proof of Theorem 7.1, that we started in Section 7.1.

Proof. Having denoted by $\mathbf{e}^{\mathcal{T}}=\mathfrak{P}_{\boldsymbol{m}, \boldsymbol{g}} \mathbb{P}_{\boldsymbol{c}}^{\mathcal{T}} \mathbf{u}-\mathbf{u}^{\mathcal{T}}$ and $e^{\mathfrak{D}}=\mathbb{P}_{m}^{\mathfrak{D}} p-p^{\mathfrak{D}}$, we have obtained the following inequality (7.6)

$$
\begin{aligned}
\left\|\nabla^{\mathfrak{D}} \mathbf{e}^{\mathcal{T}}\right\|_{2}^{2}+\left\|e^{\mathfrak{D}}\right\|_{2}^{2} \leq & C\left(\left\|\mathbf{R}_{\sigma}^{\mathbf{u}}\right\|_{2}+\left\|\mathbf{R}_{\sigma^{*}}^{\mathbf{u}}\right\|_{2}+\left\|\mathbf{R}_{\sigma}^{p}\right\|_{2}+\left\|\mathbf{R}_{\sigma^{*}}^{p}\right\|_{2}\right)\left(\left\|\nabla^{\mathfrak{D}} \mathbf{e}^{\mathcal{T}}\right\|_{2}+\left\|e^{\mathfrak{D}}\right\|_{2}\right) \\
& +C \operatorname{size}(\mathcal{T})\left(\|\mathbf{u}\|_{H^{2}}+\|\nabla p\|_{2}\right)\left(\left\|\nabla^{\mathfrak{D}} \mathbf{e}^{\mathcal{T}}\right\|_{2}+\left\|e^{\mathfrak{D}}\right\|_{2}\right) .
\end{aligned}
$$

Corollaries 7.1 and 7.2 imply

$$
\left\|\nabla^{\mathfrak{D}} \mathbf{e}^{\mathcal{T}}\right\|_{2} \leq C \operatorname{size}(\mathcal{T}) \quad \text { and } \quad\left\|e^{\mathfrak{D}}\right\|_{2} \leq C \operatorname{size}(\mathcal{T}) .
$$


Estimate of $\left\|\mathbf{u}-\mathbf{u}^{\mathcal{T}}\right\|_{2}$. We have

$$
\left\|\mathbf{u}-\mathbf{u}^{\mathcal{T}}\right\|_{2} \leq\left\|\mathbf{u}-\mathfrak{P}_{\boldsymbol{m}, \boldsymbol{g}} \mathbb{P}_{\boldsymbol{c}}^{\mathcal{T}} \mathbf{u}\right\|_{2}+\left\|\mathfrak{P}_{\boldsymbol{m}, \boldsymbol{g}} \mathbb{P}_{\boldsymbol{c}}^{\mathcal{T}} \mathbf{u}-\mathbf{u}^{\mathcal{T}}\right\|_{2} .
$$

Lemma 5.6 and the discrete Poincaré inequality (Theorem 5.2) imply

$$
\left\|\mathbf{u}-\mathbf{u}^{\mathcal{T}}\right\|_{2} \leq C \operatorname{size}(\mathcal{T})\|\nabla \mathbf{u}\|_{H^{1}}+C\left\|\nabla^{\mathfrak{D}} \mathfrak{P}_{\boldsymbol{m}, \boldsymbol{g}} \mathbb{P}_{\boldsymbol{c}}^{\mathcal{T}} \mathbf{u}-\nabla^{\mathfrak{D}} \mathbf{u}^{\mathcal{T}}\right\|_{2} .
$$

Finally, (7.9) gives the estimate of $\left\|\mathbf{u}-\mathbf{u}^{\mathcal{T}}\right\|_{2}$.

Estimate of $\left\|\nabla \mathbf{u}-\nabla^{\mathfrak{D}} \mathbf{u}^{\mathcal{T}}\right\|_{2}$. We have

$$
\begin{gathered}
\left\|\nabla \mathbf{u}-\nabla^{\mathfrak{D}} \mathbf{u}^{\mathcal{T}}\right\|_{2} \leq \\
\quad\left\|\nabla \mathbf{u}-\nabla^{\mathfrak{D}} \mathbb{P}_{\boldsymbol{c}}^{\mathcal{T}} \mathbf{u}\right\|_{2}+\left\|\nabla^{\mathfrak{D}} \mathbb{P}_{\boldsymbol{c}}^{\mathcal{T}} \mathbf{u}-\nabla^{\mathfrak{D}} \mathfrak{P}_{\boldsymbol{m}, \boldsymbol{g}} \mathbb{P}_{\boldsymbol{c}}^{\mathcal{T}} \mathbf{u}\right\|_{2} \\
+\left\|\nabla^{\mathfrak{D}} \mathfrak{P}_{\boldsymbol{m}, \boldsymbol{g}} \mathbb{P}_{\boldsymbol{c}}^{\mathcal{T}} \mathbf{u}-\nabla^{\mathfrak{D}} \mathbf{u}^{\mathcal{T}}\right\|_{2} .
\end{gathered}
$$

Finally, Lemmas 5.3, 5.4 and (7.9) imply the estimate of $\left\|\nabla \mathbf{u}-\nabla^{\mathfrak{D}} \mathbf{u}^{\mathcal{T}}\right\|_{2}$.

Estimate of $\left\|p-p^{\mathfrak{D}}\right\|_{2}$. We have

$$
\left\|p-p^{\mathfrak{D}}\right\|_{2} \leq\left\|p-\mathbb{P}_{m}^{\mathfrak{D}} p\right\|_{2}+\left\|\mathbb{P}_{m}^{\mathfrak{D}} p-p^{\mathfrak{D}}\right\|_{2}
$$

We conclude thanks to Proposition 5.4 and (7.9).

8. Numerical results. We first want to emphasize that the implementation of DDFV schemes only requires a TPFA mesh structure, that is an edge structure with references to the two neighbouring cells and to its two vertices. In particular, we never need to construct the dual mesh and the diamond mesh.

We show here some numerical results obtained on a rectangular domain $\Omega=] 0,1\left[^{2}\right.$. Error estimates are given on four different tests with a stabilization coefficient chosen to be $\lambda=10^{-3}$.

REMARK 8.1. Remark 6.1 brings out the theoretical role of $\lambda$. Reference [13] proves that if $\lambda$ is zero, the scheme is well-posed only for conformal triangle meshes with acute angles or for non-conformal square meshes, otherwise for a mesh like in Figure 8.1(a) well-posedness is open.

We present four test cases. For all of them, we give in the following the exact solution and the original mesh. In order to illustrate error estimates, the family of meshes are obtained by successive global refinement of the original mesh. In the first test, the exact solution is the Green-Taylor vortex on a quadrangle and triangle mesh (see Figure 8.1(a)). In the second one, the exact solution is a polynomial function on a non-conformal square mesh (see Figure 8.2(a)). The third and fourth tests are performed using a discontinuous viscosity function.

The exact solution $(\mathbf{u}, p)$ and the viscosity $\eta$ being chosen, we define the source term $\mathbf{f}$ and the boundary data $\mathbf{g}$ in such a way that (2.1) is satisfied. Since the pressure is smooth in our example, we compare the approximate pressure to the center-value projection of the exact pressure on $\bar{\Omega}$ :

$$
\mathbb{P}_{\boldsymbol{c}}^{\mathfrak{D}} p=\left(\left(p\left(x_{\mathcal{D}}\right)\right)_{\mathcal{D} \in \mathfrak{D}}\right)
$$

In Figures 8.1-8.4, we compare the $L^{2}$-norm of the error obtained with the DDFV scheme, for the pressure $\frac{\left\|\mathbb{P}_{\boldsymbol{c}}^{\mathfrak{D}} p-p^{\mathfrak{D}}\right\|_{2}}{\left\|\mathbb{P}_{\boldsymbol{c}}^{\mathfrak{D}} p\right\|_{2}}$, for the velocity gradient $\frac{\left\|\nabla^{\mathfrak{D}} \mathbb{P}_{\boldsymbol{c}}^{\mathcal{T}} \mathbf{u}-\nabla^{\mathfrak{D}} \mathbf{u}^{\mathcal{T}}\right\|_{2}}{\left\|\nabla^{\mathfrak{D}} \mathbb{P}_{\boldsymbol{c}}^{\mathcal{T}} \mathbf{u}\right\|_{2}}$ and for the velocity $\frac{\left\|\mathbb{P}_{\boldsymbol{c}}^{\mathcal{T}} \mathbf{u}-\mathbf{u}^{\mathcal{T}}\right\|_{2}}{\left\|\mathbb{P}_{\boldsymbol{c}}^{\mathcal{T}} \mathbf{u}\right\|_{2}}$ respectively, as functions of the size of the mesh $\operatorname{size}(\mathcal{T})$ in a logarithmic scale. The convergence rates shown on 8.1-8.4 are computed through a least square linear approximation of the computed data. 
8.1. Test 1 (Green-Taylor vortex) - Constant viscosity. Let us consider the following exact solution:

$$
\mathbf{u}(x, y)=\left(\begin{array}{c}
\frac{1}{2} \sin (2 \pi x) \cos (2 \pi y) \\
-\frac{1}{2} \cos (2 \pi x) \sin (2 \pi y)
\end{array}\right), \quad p(x, y)=\frac{1}{8} \cos (4 \pi x) \sin (4 \pi y),
$$

and the viscosity $\eta(x, y)=1$. The considered mesh is a quadrangle and triangle mesh (see Figure 8.1(a)). For smooth solution and viscosity, as predicted in Theorem 7.1, we observe

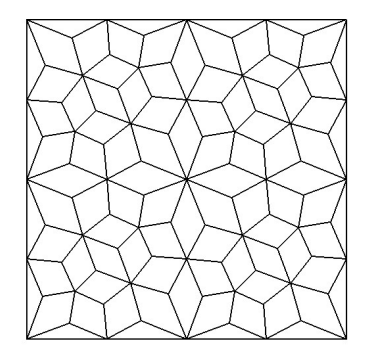

(a) Quadrangle and triangle mesh.

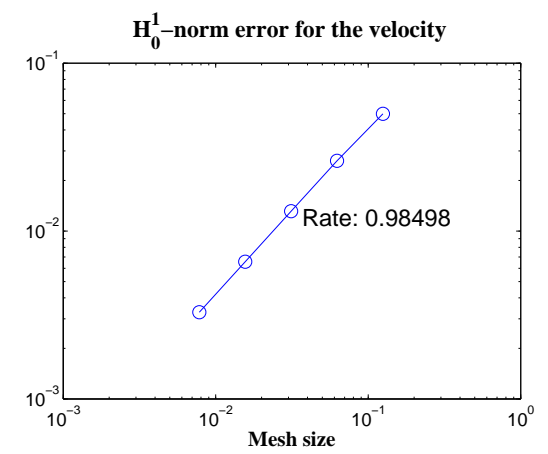

(c) $\frac{\left\|\nabla^{\mathfrak{D}} \mathbb{P}_{c}^{\mathcal{T}} \mathbf{u}-\nabla^{\mathfrak{D}} \mathbf{u}^{\mathcal{T}}\right\|_{2}}{\left\|\nabla^{\mathfrak{D}} \mathbb{P}_{\boldsymbol{c}}^{\mathcal{T}} \mathbf{u}\right\|_{2}}$

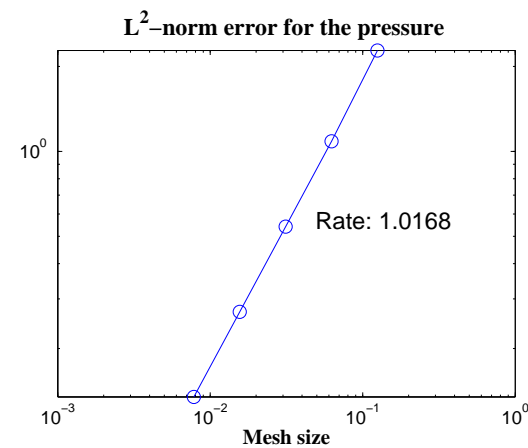

(b) $\frac{\left\|\mathbb{P}_{c}^{\mathfrak{D}} p-p^{\mathfrak{D}}\right\|_{2}}{\left\|\mathbb{P}_{c}^{\mathfrak{D}} p\right\|_{2}}$

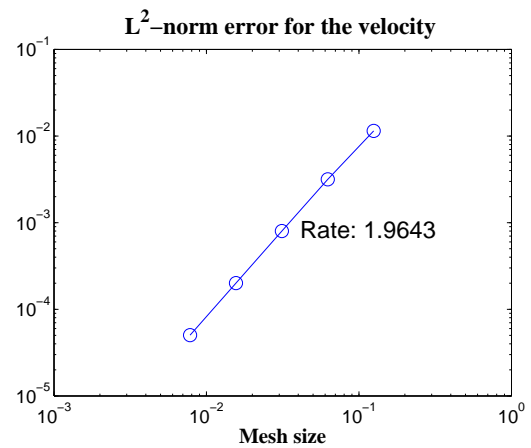

(d) $\frac{\left\|\mathbb{P}_{c}^{\mathcal{T}} \mathbf{u}-\mathbf{u}^{\mathcal{T}}\right\|_{2}}{\left\|\mathbb{P}_{\boldsymbol{c}}^{\mathcal{T}} \mathbf{u}\right\|_{2}}$

FIG. 8.1. Test 1, Green-Taylor vortex, on a quadrangle and triangle mesh.

a first order convergence for the $L^{2}$-norm of the velocity gradient and of the pressure, which seems to be optimal. We obtain a second order convergence for the $L^{2}$-norm of the velocity. This super-convergence of the $L^{2}$-norm is classical for finite volume method, however its proof still remains an open problem see [32].

For $\lambda=0$, we numerically observe the well-posedness and similar rates of convergence.

8.2. Test 2 (polynomial function) - Non constant viscosity. The exact solution on the second test are the following polynomial functions:

$$
\mathbf{u}(x, y)=\left(\begin{array}{c}
1000 x^{2}(1-x)^{2} 2 y(1-y)(1-2 y) \\
-1000 y^{2}(1-y)^{2} 2 x(1-x)(1-2 x)
\end{array}\right), \quad p(x, y)=x^{2}+y^{2}-\frac{2}{3},
$$




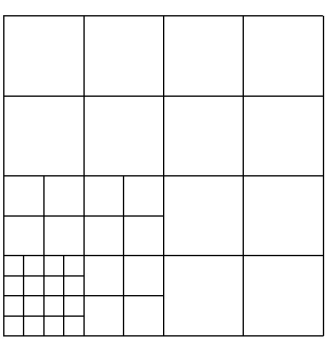

(a) Locally refined rectangular mesh.

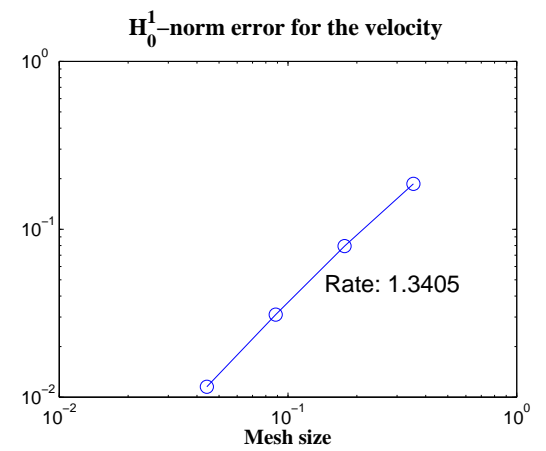

(c) $\frac{\left\|\nabla^{\mathfrak{D}} \mathbb{P}_{c}^{\mathcal{T}} \mathbf{u}-\nabla^{\mathfrak{D}} \mathbf{u}^{\mathcal{T}}\right\|_{2}}{\left\|\nabla^{\mathfrak{D}} \mathbb{P}_{\boldsymbol{c}}^{\mathcal{T}} \mathbf{u}\right\|_{2}}$

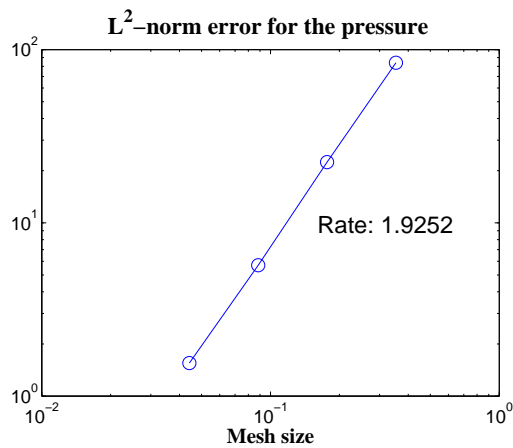

(b) $\frac{\left\|\mathbb{P}_{c}^{\mathfrak{D}} p-p^{\mathfrak{D}}\right\|_{2}}{\left\|\mathbb{P}_{\boldsymbol{c}}^{\mathfrak{D}} p\right\|_{2}}$

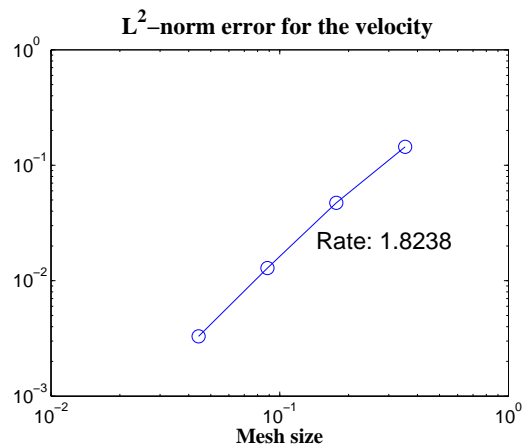

(d) $\frac{\left\|\mathbb{P}_{\boldsymbol{c}}^{\mathcal{T}} \mathbf{u}-\mathbf{u}^{\mathcal{T}}\right\|_{2}}{\left\|\mathbb{P}_{\boldsymbol{c}}^{\mathcal{T}} \mathbf{u}\right\|_{2}}$

FIG. 8.2. Test 2, polynomial function, on a non conformal rectangular mesh.

and the viscosity $\eta(x, y)=2 x+y+1$. We use the non conformal square mesh, arbitrarily locally refined on the left bottom corner, as shown on Figure 8.2(a). For the velocity, its gradient and the pressure, we numerically obtain convergence rates equal to $1.9,1.3$ and 2 respectively for the DDFV scheme. Note that the convergence rates obtained in this numerical test are greater than the theoretical one given in Theorem 7.1. This is related to some uniformity of the mesh away from the refinement area. Furthermore, let us emphasize that the convergence rate is not sensitive to the presence of non conformal control volumes.

8.3. Test 3 - Discontinuous viscosity. The exact solution is the following:

$$
\mathbf{u}(x, y)=\left(\begin{array}{c}
-\pi y \\
\sin (\pi(x-0.5))
\end{array}\right), \quad p(x, y)=2.5(x-y)
$$

Here, we consider a discontinuous viscosity:

$$
\eta(x, y)=\left\{\begin{array}{rr}
1 & \text { for } x>0.5 \\
10^{-4} & \text { else. }
\end{array}\right.
$$

We use the triangle mesh, shown on Figure 8.3(a). With this viscosity, the assumptions of Theorem 7.1 are not satisfied. Nevertheless, the numerical test shows that we have a first 


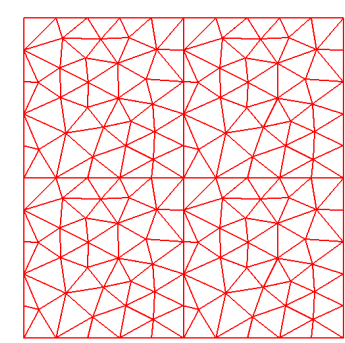

(a) Triangle mesh.

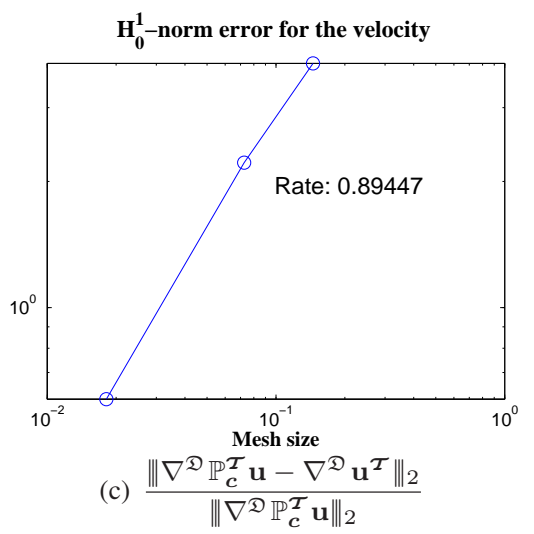

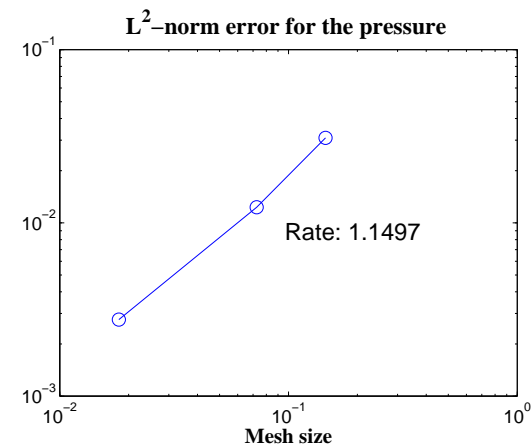

(b) $\frac{\left\|\mathbb{P}_{c}^{\mathfrak{D}} p-p^{\mathfrak{D}}\right\|_{2}}{\left\|\mathbb{P}_{\boldsymbol{c}}^{\mathfrak{D}} p\right\|_{2}}$

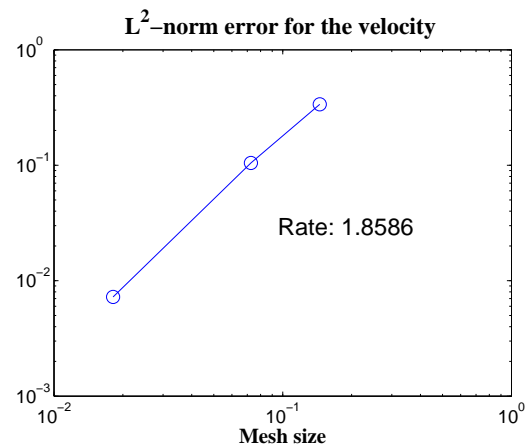

(d) $\frac{\left\|\mathbb{P}_{c}^{\mathcal{T}} \mathbf{u}-\mathbf{u}^{\mathcal{T}}\right\|_{2}}{\left\|\mathbb{P}_{\boldsymbol{c}}^{\mathcal{T}} \mathbf{u}\right\|_{2}}$

FIG. 8.3. Test 3, discontinuous viscosity on a triangle mesh.

order convergence for the velocity in $H_{0}^{1}$-norm and for the pressure in $L^{2}$-norm. It seems to come from the fact that $\mathrm{Du}$ is equal to zero across the discontinuity line of the viscosity $\{x=0.5\}$ so that the jump of viscosity does not affect the consistency properties of the numerical fluxes at the interface. We will see in the next test case that it is not always the case.

8.4. Test 4 - Discontinuous viscosity. Let us consider the following exact solution:

$$
\mathbf{u}(x, y)=\left(\begin{array}{rr}
y^{2}-0.5 y & \text { for } y>0.5 \\
10^{4}\left(y^{2}-0.5 y\right) & \text { else. } \\
0 &
\end{array}\right), \quad p(x, y)=2 x-1
$$

and the discontinuous viscosity:

$$
\eta(x, y)=\left\{\begin{array}{rr}
1 & \text { for } y>0.5 \\
10^{-4} & \text { else }
\end{array}\right.
$$

We use the non conformal quadrangle mesh, locally refined where the viscosity is discontinuous, shown on Figure 8.4(a). Once more here the assumptions on the viscosity of Theorem 7.1 are not satisfied and the symmetric part of the gradient $\mathrm{Du}$ is discontinuous across the 


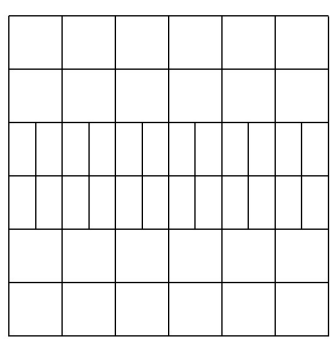

(a) Non conformal quadrangle mesh.

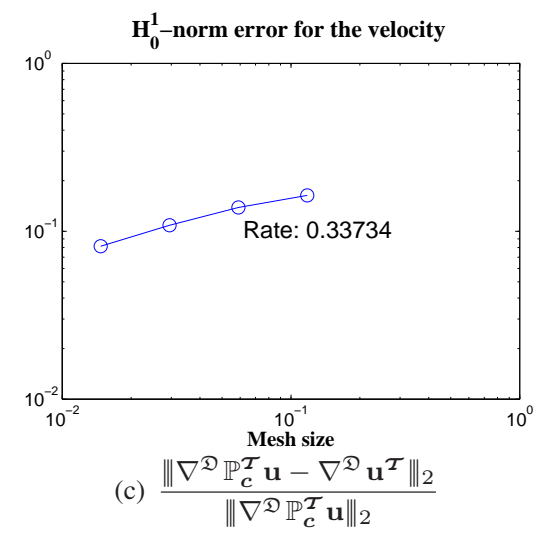

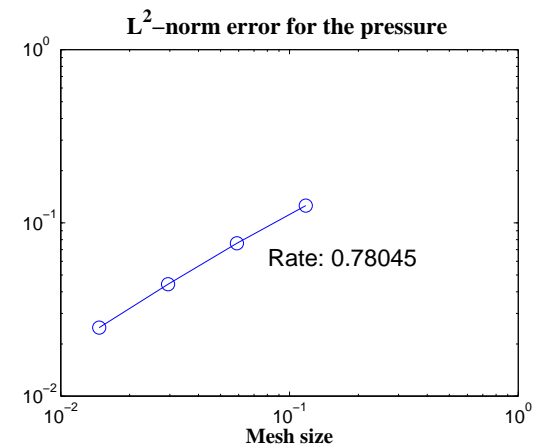

(b) $\frac{\left\|\mathbb{P}_{c}^{\mathfrak{D}} p-p^{\mathfrak{D}}\right\|_{2}}{\left\|\mathbb{P}_{c}^{\mathfrak{D}} p\right\|_{2}}$

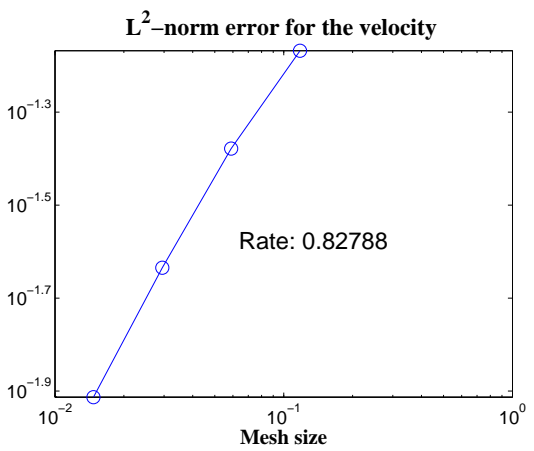

(d) $\frac{\left\|\mathbb{P}_{c}^{\mathcal{T}} \mathbf{u}-\mathbf{u}^{\mathcal{T}}\right\|_{2}}{\left\|\mathbb{P}_{\boldsymbol{c}}^{\mathcal{T}} \mathbf{u}\right\|_{2}}$

FIG. 8.4. Test 4, discontinuous viscosity on a non conformal quadrangle mesh.

interface $\{y=0.5\}$. We observe that the scheme is still convergent even if we have lost the first order convergence for the DDFV scheme, as expected.

In this test case, the discontinuities of the viscosity must be taken into account by the scheme to overcome this consistency defect. We propose and analyse such a modification of the present scheme in [29], following the strategy of [5].

9. Conclusion. In this article, we propose stabilized DDFV schemes with Dirichlet boundary conditions for the Stokes problem with variable viscosity. The DDFV scheme is proved to be well-posed and to be first order convergent in the $L^{2}$-norm for the velocity gradient, as well as for the velocity and for the pressure. These results are proved in the case where the viscosity is smooth on the whole domain $\Omega$. When the viscosity is no more smooth, we still numerically observe the convergence of the DDFV scheme. Nevertheless, the method is no more first order convergent.

In practice, the viscosity may present discontinuities across some interfaces, in multiphase flows, for instance. Such a viscosity function is no more Lipschitz continuous on the whole domain $\Omega$. For anisotropic scalar elliptic problems, in presence of discontinuities, a suitable modification of the discrete gradient allows the authors of [5] to recover the first order convergence. This approach can be adapted to the present framework for the Stokes interface problem. We proved in [29] that the modified DDFV scheme presents a better consistency of the fluxes near the places where discontinuities of the viscosity occur. Finally, the modified 
scheme is proved to be well-posed and first order convergent on 2D general meshes, even for a discontinuous viscosity.

Acknowledgments. The author want to express its gratitude to Franck Boyer and Florence Hubert for their support during the preparation of this work.

\section{REFERENCES}

[1] B. Andreianov, M. Bendahmane, and K. Karlsen. A gradient reconstruction formula for finite-volume schemes and discrete duality. In R. Eymard and J. M. Herard, editors, Proceedings of Finite Volumes for Complex Applications V. Wiley, 2008.

[2] B. Andreianov, F. Boyer, and F. Hubert. Discrete duality finite volume schemes for Leray-Lions type elliptic problems on general 2D-meshes. Num. Meth. for PDEs, 23(1):145-195, 2007.

[3] B. Andreianov, M. Gutnic, and P. Wittbold. Convergence of finite volume approximations for a nonlinear elliptic-parabolic problem: a "continuous" approach. SIAM J. Numer. Anal., 42(1):228-251 (electronic), 2004.

[4] F. Boyer and P. Fabrie. Eléments d'analyse pour l'étude de quelques modèles d'écoulements de fluides visqueux incompressibles, volume 52 of Mathématiques \& Applications (Berlin) [Mathematics \& Applications]. Springer-Verlag, Berlin, 2006.

[5] F. Boyer and F. Hubert. Finite volume method for $2 \mathrm{~d}$ linear and nonlinear elliptic problems with discontinuities. SIAM J. Numer. Anal., 46(6):3032-3070, 2008.

[6] F. Boyer, F. Hubert, and S. Krell. Non-overlapping Schwarz algorithm for solving 2d m-DDFV schemes. 2009. to appear in IMA J. N. A., Available online at doi:10.1093/imanum/drp001.

[7] F. Brezzi and M. Fortin. Mixed and hybrid finite element methods, volume 15 of Springer Series in Computational Mathematics. Springer-Verlag, New York, 1991.

[8] F. Brezzi and J. Pitkäranta. On the stabilization of finite element approximations of the Stokes equations. In Efficient solutions of elliptic systems (Kiel, 1984), volume 10 of Notes Numer. Fluid Mech., pages 11-19. Vieweg, Braunschweig, 1984

[9] Y. Coudière and F. Hubert. A 3D discrete duality finite volume method for nonlinear elliptic equation. Podbanske, Slovaquie, 2009. Proceedings of Algorithmy, http://hal.archives-ouvertes.fr/hal-00356879/fr/.

[10] Y. Coudière and G. Manzini. The discrete duality finite volume method for convection-diffusion problems. SIAM Journal on Numerical Analysis, 47(6):4163-4192, 2010. Available online at http://dx.doi.org/10.1137/080731219.

[11] Y. Coudière, C. Pierre, O. Rousseau, and R. Turpault. A 2D/3D discrete duality finite volume scheme. Application to ECG simulation. Int. J. Finite Vol., 6(1):24, 2009.

[12] Y. Coudière, J.-P. Vila, and P. Villedieu. Convergence rate of a finite volume scheme for a two-dimensional convection-diffusion problem. M2AN Math. Model. Numer. Anal., 33(3):493-516, 1999.

[13] S. Delcourte. Développement de méthodes de volumes finis pour la mécanique des fluides. $\mathrm{PhD}$ thesis, http://tel.archives-ouvertes.fr/tel-00200833/fr/, 2007.

[14] S. Delcourte, K. Domelevo, and P. Omnes. A discrete duality finite volume approach to Hodge decomposition and div-curl problems on almost arbitrary two-dimensional meshes. SIAM J. Numer. Anal., 45(3):11421174 (electronic), 2007.

[15] K. Domelevo and P. Omnes. A finite volume method for the Laplace equation on almost arbitrary twodimensional grids. M2AN Math. Model. Numer. Anal., 39(6):1203-1249, 2005.

[16] J. Droniou. Finite volume schemes for fully non-linear elliptic equations in divergence form. M2AN Math. Model. Numer. Anal., 40(6):1069-1100 (2007), 2006.

[17] J. Droniou and R. Eymard. A mixed finite volume scheme for anisotropic diffusion problems on any grid. Numer. Math., 105(1):35-71, 2006.

[18] R. Eymard, T. Gallouët, and R. Herbin. Finite volume methods. In Handbook of numerical analysis, Vol. VII, Handb. Numer. Anal., VII, pages 713-1020. North-Holland, Amsterdam, 2000.

[19] R. Eymard, T. Gallouët, and R. Herbin. A cell-centered finite-volume approximation for anisotropic diffusion operators on unstructured meshes in any space dimension. IMA J. Numer. Anal., 26(2):326-353, 2006.

[20] R. Eymard, R. Herbin, and J. C. Latché. On a stabilized colocated finite volume scheme for the Stokes problem. M2AN Math. Model. Numer. Anal., 40(3):501-527, 2006.

[21] R. Eymard, R. Herbin, and J. C. Latché. Convergence analysis of a colocated finite volume scheme for the incompressible Navier-Stokes equations on general 2D or 3D meshes. SIAM J. Numer. Anal., 45(1):1-36 (electronic), 2007.

[22] V. Girault and P.-A. Raviart. Finite element methods for Navier-Stokes equations, volume 5 of Springer Series in Computational Mathematics. Springer-Verlag, Berlin, 1986. Theory and algorithms.

[23] F. Harlow and J. Welch. Numerical calculation of time-dependent viscous incompressible flow of fluid with free surface. The physics of fluids, 8(12):2182-2189, 1965. 
[24] R. Herbin and F. Hubert. Benchmark on discretization schemes for anisotropic diffsion problems on general grids. In R. Eymard and J. M. Herard, editors, Proceedings of Finite Volumes for Complex Applications $V$. Wiley, 2008.

[25] F. Hermeline. A finite volume method for the approximation of diffusion operators on distorted meshes. $J$. Comput. Phys., 160(2):481-499, 2000.

[26] F. Hermeline. Approximation of diffusion operators with discontinuous tensor coefficients on distorted meshes. Comput. Methods Appl. Mech. Engrg., 192(16-18):1939-1959, 2003.

[27] F. Hermeline. Approximation of 2-D and 3-D diffusion operators with variable full tensor coefficients on arbitrary meshes. Comput. Methods Appl. Mech. Engrg., 196(21-24):2497-2526, 2007.

[28] R. B. Kellogg and J. E. Osborn. A regularity result for the Stokes problem in a convex polygon. J. Functional Analysis, 21(4):397-431, 1976.

[29] S. Krell. Finite volume method for general multifluid flows governed by the interface Stokes problem. 2010. in preparation.

[30] S. Krell and G. Manzini. The Discrete Duality Finite Volume method for the Stokes equation on 3D polyhedral meshes. preprint, 2009. Available online at http://hal.archives-ouvertes.fr/hal-00448465/fr/.

[31] R. A. Nicolaides. Analysis and convergence of the MAC scheme. I. The linear problem. SIAM J. Numer. Anal., 29(6):1579-1591, 1992.

[32] P. Omnes. On the second-order convergence of finite volume methods for the Laplace equation on DelaunayVoronoi meshes. 2010. Preprint, Available online at http://hal-cea.archives-ouvertes.fr/cea-00430941/fr/.

[33] R. Temam. Navier-Stokes equations. Theory and numerical analysis. North-Holland Publishing Co., Amsterdam, 1977. Studies in Mathematics and its Applications, Vol. 2. 